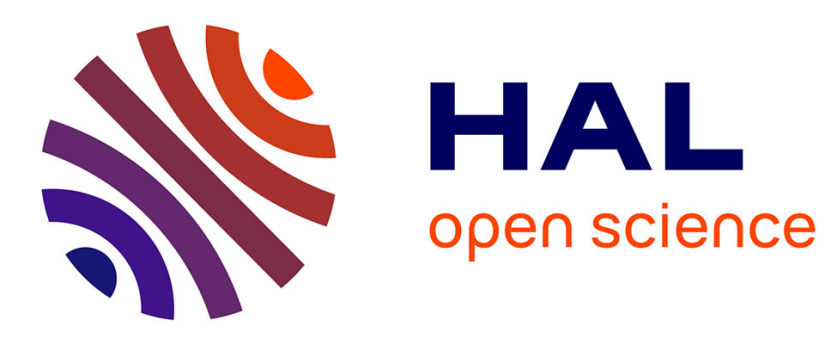

\title{
Transparent Heaters: A Review
}

Dorina T. Papanastasiou, Amélie Schultheiss, David Muñoz-Rojas, Caroline

Celle, Alexandre Carella, Jean-Pierre Simonato, Daniel Bellet

\section{To cite this version:}

Dorina T. Papanastasiou, Amélie Schultheiss, David Muñoz-Rojas, Caroline Celle, Alexandre Carella, et al.. Transparent Heaters: A Review. Advanced Functional Materials, 2020, pp.1910225. 10.1002/adfm.201910225. hal-02513426

\section{HAL Id: hal-02513426 \\ https://hal.science/hal-02513426}

Submitted on 7 Jan 2021

HAL is a multi-disciplinary open access archive for the deposit and dissemination of scientific research documents, whether they are published or not. The documents may come from teaching and research institutions in France or abroad, or from public or private research centers.
L'archive ouverte pluridisciplinaire HAL, est destinée au dépôt et à la diffusion de documents scientifiques de niveau recherche, publiés ou non, émanant des établissements d'enseignement et de recherche français ou étrangers, des laboratoires publics ou privés. 


\section{WILEY-VCH}

\section{Transparent Heaters: A Review}

2 Dorina T. Papanastasiou, Amélie Schultheiss, David Muñoz-Rojas*, Caroline Celle, Alexandre

3 Carella, Jean-Pierre Simonato*, Daniel Bellet*

$4 *$ corresponding authors

5 Keywords: transparent electrode, Joule heating, film heater, composite, stability

6

7 D.T. Papanastasiou, Dr. D. Muñoz-Rojas, Pr. D. Bellet

8 Univ. Grenoble Alpes, CNRS, Grenoble INP, LMGP, F- 38000 Grenoble, France

9 Email : david.munoz-rojas@ grenoble-inp.fr; daniel.bellet@ grenoble-inp.fr

10 A. Schultheiss, Dr. C. Celle, Dr. A. Carella, Dr. J.-P. Simonato

11 Univ. Grenoble Alpes, CEA, LITEN, DTNM, 17 avenue des Martyrs, F-38054 Grenoble,

12 France

13 Email: jean-pierre.simonato@cea.fr

14

15 


\section{WILEY-VCH}

\section{Short summary}

17 Transparent heaters $(\mathrm{TH})$ have attracted intense attention from both scientific and industrial

18 actors due to the key role they play in many technologies, including smart windows, deicers,

19 defoggers, displays, actuators and sensors. While transparent conductive oxides have

20 dominated the field for the past five decades, a new generation of THs based on nanomaterials

21 has led to new paradigms in terms of applications and prospects in the past years. Here we will

22 review the most recent developments and strategies to improve the properties, stability and

23 integration of these new THs.

25

\section{Graphical abstract}

27

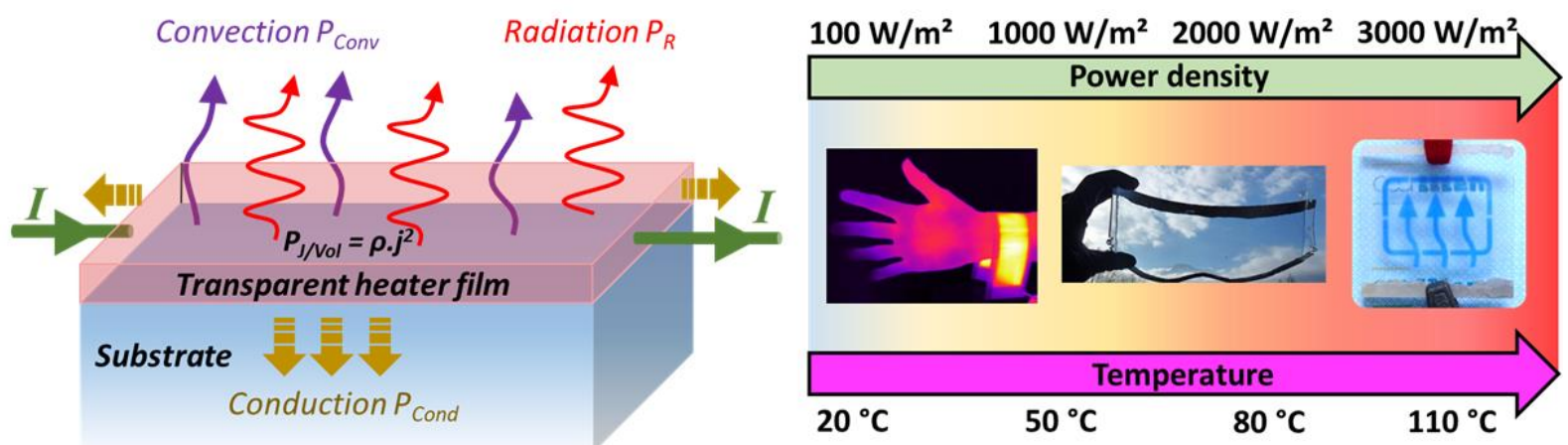




\section{WILEY-VCH}

\section{Introduction}

32 inertia of thin TH films.

Transparent heaters (THs) are visually transparent devices that contain electrically conductive layers. When an electrical current flows across the transparent heater, it generates heat thanks to the Joule effect. This heat can be efficiently used in many devices. Consequently, numerous applications are concerned by THs, and the associated TH market is growing fast, comprising many types of devices (smart windows, deicers, defoggers, displays, thermotherapy pads, sensors...) for different industrial sectors (transport, buildings, healthcare, sport...). For instance, THs can provide the warmth required to extend the operating temperature of liquid crystal displays (LCDs) in cold environments or can increase the temperature for anti-fogging systems, anti-icing, deicing of optics and optical displays (very useful for transportation). Other applications include the heating of specific industrial processes, of microfluidic chips, of kiosks and handheld devices. ${ }^{[1]}$ Display technology and smart windows (used in transport or for interior architecture) are among the fastest growing markets related to THs. Importantly, defrosting windows in airplanes enables high-altitude flying. ${ }^{[2]}$ Smart windows can reduce energy loss in buildings, which currently account for over $40 \%$ of the energy consumed. This works thanks to special functional coatings which can regulate solar energy in response to heat (thermochromic material). ${ }^{[3,4]}$ This heat can be generated by THs within smart windows. ${ }^{[5]}$ While the required optical transparency depends upon the specific application, in this review we have considered THs having an optical transparency of at least 50\%.

The fast control of temperature via the applied current or voltage makes THs a generally better choice when compared to conventional heating elements. This is enabled by the small thermal

The only technology used for TH before 1995 was based on transparent conductive oxides (TCOs), which were also broadly used as transparent electrodes in many applications including photovoltaics $^{[6]}$, smart windows ${ }^{[7]}$, efficient lighting ${ }^{[8]}$ or displays. ${ }^{[2,7,9,10]}$ The three main TCO 


\section{WILEY-VCH}

materials are indium tin oxide (ITO $)^{[11-13]}$, the most well-known and used TCO, fluorine-doped tin oxide $(\mathrm{FTO})^{[14,15]}$ and aluminum-doped zinc oxide (AZO). ${ }^{[16-19]}$ TCOs are generally degenerately-doped n-type semiconductors which exhibit a high electrical conductivity of $10^{3}$ $10^{4} \mathrm{~S} / \mathrm{cm} .{ }^{[9]}$ They are often fabricated as thin films of approximately $300 \mathrm{~nm}$ thickness in order to show high transparency ( $90 \%)$. While TCOs can exhibit good or even excellent performances in terms of the trade-off between electrical conductivity and optical transparency in the visible range, their two main drawbacks concern their non-flexibility (due to their ceramic nature) and their intrinsic low optical transmittance in the near-infrared (NIR) spectrum due to plasmonic absorption ${ }^{[13,20]}$. Therefore, great attention has been devoted to flexible and transparent electrodes ${ }^{[21]}$ or heaters for three main reasons: $\mathrm{i} /$ the emergence of new-generation devices having flexible or nonplanar substrates, ii/ the potential scarcity of indium for ITO and iii/ the lack of transparency in the NIR. For some applications, even flexibility is not sufficient since stretchability could be a prerequisite, like for the integration of THs within wearable electronics. ${ }^{[22]}$ For example, flexible but also stretchable THs can be used in thermotherapy ${ }^{[23,24]}$, a popular treatment in physiotherapy which is particularly useful for the treatment of joint injuries and pain. For such applications, THs should also be soft and thin (and therefore light) so that comfort is not compromised.

It is also worth noting that even for non-flexible devices, we are looking for high throughput fabrication methods which are compatible with an economically viable technology, like the roll-to-roll (R2R) process. Indeed, R2R is a fast processing method associated with little or no loss of material, and which requires that both substrate and active layers be flexible. ${ }^{[25,26]}$ Therefore TCOs themselves are not compatible with such low-cost methods, except when integrated as very thin films with other materials such as oxides or metals, and thus alternative THs need to be developed.

Several emerging materials have recently been investigated for $\mathrm{TH}$ applications, including carbon-based nanomaterials, and mainly carbon nanotubes $(\mathrm{CNT})^{[27]}$ and graphene ${ }^{[28]}$. These 


\section{WILEY-VCH}

new materials are rather cheap and abundant and could therefore follow the fast-growing industrial demand. Moreover, THs made of these building blocks are flexible and can be processed in solution. In parallel, since 2009 metallic nanowire (MNW) networks have started to be more systematically investigated, notably following the seminal articles on: i) the synthesis of MNWs by the groups of Sun ${ }^{[29]}$ and Wiley ${ }^{[30-32]}$, among others; ii) the study of the physical properties of MNW networks by Coleman's group ${ }^{[33,34]}$; and iii) the integration into efficient devices by Pei's group. ${ }^{[35,36]}$ Several recent reviews have summarized the main properties, challenges and integration of MNW networks ${ }^{[20,37-41]}$. The first article showing that silver nanowire (AgNW) networks could act as efficient THs was published in 2012 by Simonato's group. ${ }^{[42]}$ As shown below, this ground-breaking paper was followed by many other studies focused on the physics of THs based on MNW networks ${ }^{[43]}$, on the integration of this type of TH into various devices ${ }^{[22,44,45]}$ as well as on a better understanding and enhancement of these THs. ${ }^{[46-48]}$ More recently, great progress has also been reported on conductive polymers which can now be considered as very efficient materials for THs. ${ }^{[49]}$ For instance, Simonato's group ${ }^{[50]}$ has also reported the very first efficient TH exclusively based on thin films of conducting polymers.

While many reports show an intense research activity on each of these TH technologies, there is also active research on multi-layered materials or nanocomposites which can combine the advantages of different materials. For instance, Ko et al. showed that high-performance and flexible R2R sputtered ITO/Ag/ITO multilayers can exhibit a high optical transmittance (88.2\%),low sheet resistance ( $3 \Omega / \mathrm{sq})$ and can be efficiently integrated in a $\mathrm{TH}$ device. ${ }^{[51]}$ Similarly, Park et al. reported that ITO/Cu/ITO multilayer materials can also show promising electrical and optical performances and good TH integration. ${ }^{[52]}$ The different technologies will be thoroughly discussed in section 3 of this review.

The main scientific and technological features associated with THs can be listed as follows: thermal response time, steady state temperature, homogeneity, mechanical properties (under 


\section{WILEY-VCH}

109 bending and/or stretching tests), cycling/thermal/electrical/environmental stability including ageing, electrical and optical properties, fabrication process of the $\mathrm{TH}$ (the temperature being a

111 potential issue for some applications), the material used, $\mathrm{TH}$ size and, finally, the overall

112 fabrication cost, including both materials and processes.

113 Expected requirements associated to the TH can drastically differ depending on the targeted

114 applications. For instance, smart windows, windscreens or displays require that the THs and

115 their associated substrates exhibit high optical clarity, i.e. high optical transparency and low 116 haziness (defined as the ratio between diffuse and total transmittance of light). Optical 117 engineering related to THs notably concerns matching the optical index to improve reflection 118 and transmission properties in the desired wavelength band (i.e. visible and/or NIR spectra).

119 The thermal response time is also a crucial parameter. The targeted steady state temperature of

120 THs can be very different depending on the application. For smart windows applications, the

121 thermochromic transition (i.e. metal-insulator transition) associated to hybridized

$122 \mathrm{VO}_{2} /$ Graphene is about $60{ }^{\circ} \mathrm{C}^{[53]}$. A low temperature is generally required for de-icing or

123 defogging uses (below $30{ }^{\circ} \mathrm{C}$ ). Some other applications need much higher temperatures, like

124 fast defrosting in automotive or for gas sensors (see section 4 of this review). In these cases the

125 chosen material for the TH should exhibit a very good thermal and electrical stability. TH

126 stability can be a serious issue, especially when numerous cycles are foreseen during the

127 lifetime of the device. It is worth noting that stability is related to several other aspects,

128 including potential cycling/thermal/time/electrical/environmental issues. These crucial aspects

129 will also be addressed in this review. The homogeneity of THs plays a key role for many

130 applications. For example, this is essential for eye comfort when THs are used in windscreens,

131 while hot spots can potentially be at the origin of the TH degradation. ${ }^{[54]}$

132 As for all industrial devices, cost is overwhelmingly important to propel the commercialization

133 and large industrial production (e.g., adding a TH within a ski or motorcycle helmet visor should

134 only cost a few euros), with only a handful of exceptions like spatial applications. 


\section{WILEY-VCH}

135 This review is organized as follows. The main physical mechanisms associated with THs are

136 presented in part 2.1, as well as simple physical models developed to better understand $\mathrm{TH}$

137 behavior. The main experimental methods currently used to investigate and explore the critical

138 properties of THs will be described in part 2.2. Stability issues are thoroughly considered in

139 section 2.3, from their physical origin to the solution for stability enhancement. Each $\mathrm{TH}$

140 technology (i.e. associated to specific material) is detailed. A thorough comparison and critical

141 discussion of the different active materials are reported in section 3. Part 4 focuses on the

142 integration of THs in different devices with specific associated requirements and performances.

143 Finally, part 5 gives an overview of the main challenges that lie ahead and the main prospects

144 related to THs in terms of better physical understanding, better performances and stability, as

145 well as their future integration into industrial devices.

2 Principle of transparent heaters (THs):

\subsection{Introduction to the thermal physics of THs}

As stated above, THs are based on the Joule effect. Joule's law, experimentally demonstrated by J.P. Joule (1818-1889), states that when an electrical current $I$ crosses a homogeneous conductive material with an electrical resistance $R$, the amount of heat released is equal to $I^{2} R$ per unit of time. The power dissipated in the material is directly related to the achieved steadystate temperature, through a balance between the Joule effect and heat loss. These thermal losses are schematically presented in Figure 1a: they correspond to the total heat transfer from the TH and are associated to three main physical origins: i/ thermal conduction to the substrate or through any conducting connections, ii/ convection to the surrounding air and iii/ radiation emitted from the hot surfaces. Figure $1 \mathrm{~b}$ exhibits some TH applications (medical, defogging, thermochromic), with characteristic values of temperature and areal power density. Several TH 


\section{WILEY-VCH}

160 technologies do exist, and their corresponding temperature ranges are roughly presented in

161 Figure $1 \mathrm{~b}$ in dashed lines. That being said, these temperatures depend upon the $\mathrm{TH}$ fabrication process and the experimental conditions under which the $\mathrm{TH}$ is used for a given application.

163 Figure 1c reports the typical performance of the main $\mathrm{TH}$ technologies in terms of optical

164 transparency $\operatorname{Tr}$ (the substrate transparency being subtracted) versus the electrical sheet

165 resistance $R_{s h}$. The ongoing efforts by the community are focused on fabricating THs which

166 are, simultaneously, as transparent and as conductive as possible (i.e. with a low sheet resistance). There is a trade-off between these two properties since for a homogeneous transparent conductive layer, the thicker the layer the lower the sheet resistance, but also the lower the optical transparency. This trade-off can be approached thanks to a figure of merit $(F o M)$. For transparent electrodes (TEs), two main figures of merit have been proposed thus far. The first one was derived by Haacke et al. in 1976 who proposed to consider the ratio between the $10^{\text {th }}$ power of the total optical transmittance $\operatorname{Tr}$ divided by the sheet resistance $R_{s h}$ : $\operatorname{Tr}^{10} / R_{s h}{ }^{[55]}$ Haacke's FoM is very often used when dealing with transparent electrodes applications (such as photovoltaic or displays) but also for TH investigations. Another figure of merit was later derived by Coleman's group, who considered the ratio between the electrical conductivity and the optical conductivity (equal to the optical absorption coefficient divided by

177 the free space impedance $\mathrm{Z}_{0}$ (the latter is equal to: $1 /\left(c . \varepsilon_{0}\right)$, where $c$ is the speed of light and $\varepsilon_{0}$ 178 the permittivity of free space). ${ }^{[33]}$ Both these FoMs are used as guides for comparing TEs and 179 THs. Although they make physical sense for homogeneous and continuous thin layers, they

180 have to be considered with more caution for non-homogeneous layers such as CNT networks, 181 metallic grids, or MNW networks. For example, in the case of very long 1D conductive objects, 182 a very high FoM could be obtained even if it does not present high homogeneity or usefulness

183 for device integration. Different values of Haacke's FoM are plotted in Figure 1c, showing that 184 there is a good variety of $\mathrm{TH}$ technologies that exhibit very good electrical and optical 185 properties, and that are therefore well suited for $\mathrm{TH}$ integration. Other parameters of $\mathrm{TH}$ 


\section{WILEY-VCH}

186 applications have to be taken in consideration as well; for instance, graphene appears to be less

187 conductive and therefore it will not be used alone for fast $\mathrm{TH}$ operation, as discussed in section

188 3.5.4. Chen et al. reported recently copper grid TH with an outstanding Haacke's FoM over

$7820.10^{-3} \Omega /$ sq (see Figure 1c), and a fast heating rate at low voltage. ${ }^{[56]}$ This clearly illustrates

190 the efficiency of such technology as TH.

191 Figure 1d exhibits the time-dependent temperature of graphene-based THs with two different

192 doping agents, and an ITO-based TH for the sake of comparison. ${ }^{[57]}$ During the "ON" voltage

193 state, the classical time-dependent behavior shows a linear increase at first, followed by a

194 saturation to a steady-state temperature. The three different materials used have different initial

$195 R_{s h}$ and therefore exhibit different steady-state temperatures for the same applied voltage (here

19612 volts). ${ }^{[57]}$

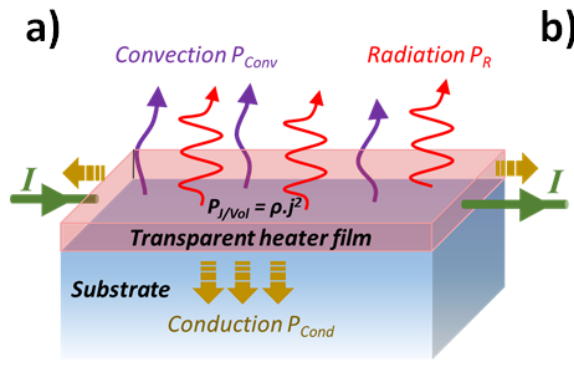

d)

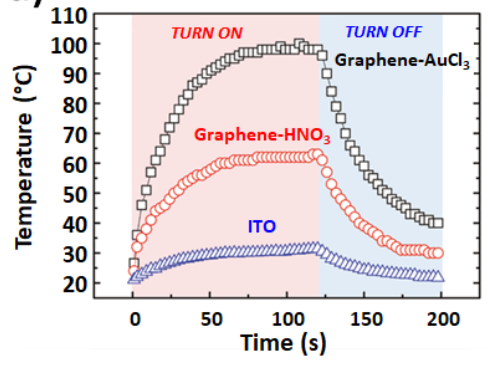

b)

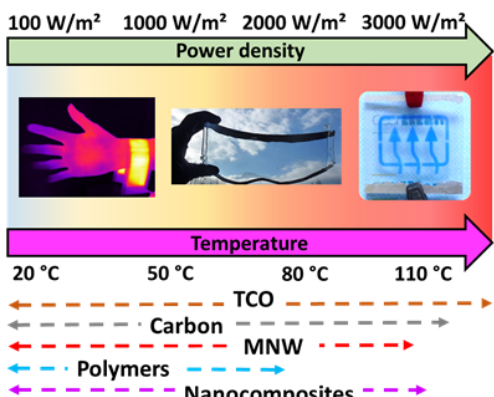

e)

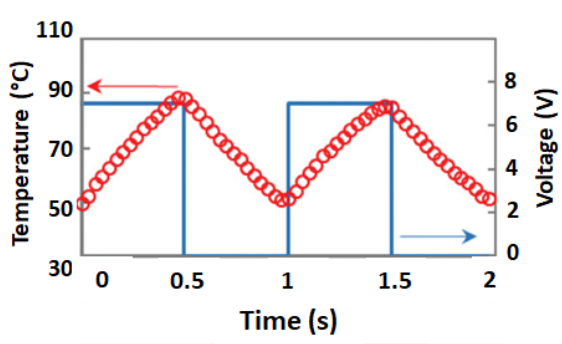

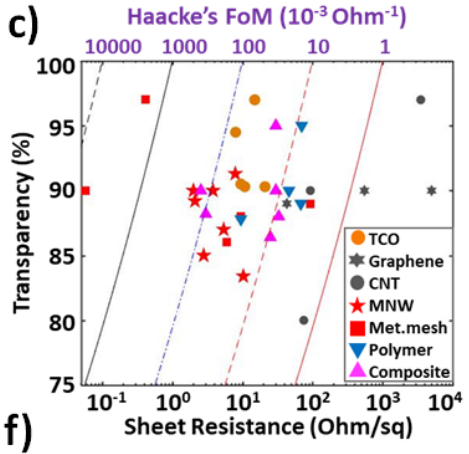

f)

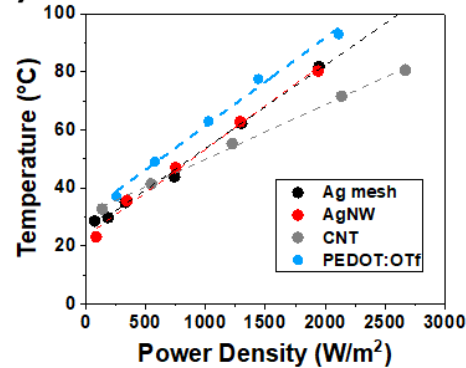

Figure 1. Main features of THs. a) Schematic of a TH: the thin TH is crossed by an electrical current, leading to heat production thanks to the Joule effect. A steady state is reached once this generated heat is equal to the sum of the heat losses shown with different colors. b) Main application domains of THs versus areal power density or temperature required. The arrows below represent roughly the typical maximum steady-state temperatures for the main TH technologies. c) Optical transparency versus sheet resistance diagram for different TH technologies based on: transparent conductive oxides (TCO) ${ }^{[14,33,58-}$ ${ }^{60]}$, carbon nanotubes $(\mathrm{CNT})^{[27,33,61]}$, graphene ${ }^{[57,62,63]}$, metallic nanowire $(\mathrm{MNW})$ networks ${ }^{[64-70]}$, metallic grids-meshes ${ }^{[56,71-76]}$, conductive polymers ${ }^{[49,50,77,78]}$, nanocomposites ${ }^{[22,48,51,79-81]}$. The dashed lines correspond to different values of Haacke's FoM: 10000, 1000, 100, 10, 1 times $10^{-3} \Omega / \mathrm{sq}^{-1}$. d) Time- 


\section{WILEY-VCH}

transitional and steady-state regimes. The optical transmittance of ITO is $95.6 \%$ and those of graphenebased THs are $88.5 \%$ and $87.5 \%$, respectively for $\mathrm{AuCl}_{3}$ and $\mathrm{HNO}_{3}$ doping protocols. Reproduced with permission. ${ }^{[57]}$ Copyright 2011, American Chemical Society. e) Time-dependence of temperature during voltage cycling showing a very fast thermal response when a TH (here composed of a carbon nanotube network) is deposited on a very thin substrate $\left(12 \Omega . \mathrm{m}\right.$ thick mica) ${ }^{[61]}$, in agreement with eq.4. Reproduced with permission. ${ }^{[61]}$. Copyright 2017, AIP Publishing f) Areal power density dependence of the stabilized temperatures measured for different TH technologies: a linear dependency is generally observed at least for temperatures lower than $100{ }^{\circ} \mathrm{C}$ (data for $\mathrm{TH}$ based on $\mathrm{AgNW}^{[46]}, \mathrm{Ag}$ mesh ${ }^{[82]}$, $\mathrm{CNT}^{[61]}$, PEDOT:OTf ${ }^{[50]}$ ). The slope appears rather similar for the different $\mathrm{TH}$ technologies.

Sorel et al. ${ }^{[43]}$ gave a comprehensive and thorough description of the theory of Joule heating in a TH in general, and focused in more detail on THs based on silver nanowire networks. But similar approaches can also be found for other types of TH technologies, such as graphene ${ }^{[83]}$.In general, the main key parameters for a $\mathrm{TH}$ are the steady-state temperature $T_{\text {stab }}$, and the time needed to approach $T_{\text {stab }}$. Most THs are supposed to reach the desired temperature by using a rather low voltage $(<12 \mathrm{~V}$ for standard uses) and after a relatively short response time (i.e. from a few seconds to a few minutes).

Considering that the temperature is uniform across the whole $\mathrm{TH}$, the energy balance at a given time $t$ can be written as follows (conduction losses due to external parts of the system are neglected ${ }^{[43]}$ :

$I^{2} R=\left(m_{1} C_{1}+m_{2} C_{2}\right) \frac{d T(t)}{d t}+A\left(h_{1}+h_{2}\right)\left(T(t)-T_{0}\right)+\sigma A\left(\varepsilon_{1}+\varepsilon_{2}\right)\left(T(t)^{4}-T_{0}{ }^{4}\right)$

For each parameter, the indexes 1 and 2 correspond to the TH and the substrate, respectively; the instantaneous sample temperature is $T(t)$, while the ambient temperature is $T_{0}$. In eq. 1 , the term on the left is the dissipated heat per unit of time. The first term on the right is the sum of the heat per unit of time associated to the TH and to the substrate (transported via thermal conduction). This heat is responsible for the temperature elevation of the sample through the specific heat capacities $C$, with $m_{1}$ and $m_{2}$ being the mass of the TH itself and of the substrate respectively. The second term represents the heat losses per unit of time by convection, with $h_{1}$ and $h_{2}$ being the convective heat-transfer coefficients and $A$ the film area, assumed to be equal to the substrate area. Finally, the third term stands for the heat losses by radiation, where $\sigma$ is 


\section{WILEY-VCH}

238 the Stefan-Boltzmann constant and $\varepsilon_{1}$ and $\varepsilon_{2}$ the emissivity of the $\mathrm{TH}$ and of the substrate, respectively. Considering small temperature rises, allowing a linear expansion of eq.1 (the non-

240 linear term associated to radiation losses being neglected) and with the hypothesis that the TH 241 has a much lower heat capacity compared to the substrate $\left(m_{1} C_{1} \ll m_{2} C_{2}\right)$, the energy balance 242 then has the following analytical formula ${ }^{[43]}$ :

$$
\Delta T(t)=T(t)-T_{0} \approx \frac{1}{\alpha} \frac{I^{2} R}{A}\left[1-\exp \left(-\frac{\alpha}{m_{2} C_{2} / A} t\right)\right]
$$

244 where $\alpha$ is the heat transfer constant, which takes into account the parameters of heat losses 245 expressed as follows:

$\alpha=\left(h_{1}+h_{2}\right)+4\left(\varepsilon_{1}+\varepsilon_{2}\right) \sigma T_{0}^{3}$

247 Eq. (2) shows that there is a transitional state for a short time, i.e. for a small $t$ compared to the 248 characteristic time $\tau$, while for times much larger than $\tau$, a steady-state temperature is achieved 249 and the saturation temperature is then equal to: $\frac{1}{\alpha} \frac{I^{2} R}{A}$. The expression of $\tau$ is given by:

$\tau=\frac{m_{2} C_{2}}{\alpha A}=\frac{\rho_{\text {sub }} t_{\text {sub }} C_{\text {sub }}}{\alpha}$

where $\rho_{s u b}, t_{s u b}$ and $C_{s u b}$ are, respectively, the density, thickness and specific heat (per unit of volume) of the substrate. After a time $t=\tau$, the temperature reaches $63 \%$ of the saturation temperature. Another characteristic time, called the response time, is also often used in the

254 literature: it is defined as the time required to reach $90 \%$ of the steady-state temperature, its 255 value being equal to $\operatorname{Ln}(0.1) \tau$, i.e. $2.3 \tau$.

The existence of both transitional and steady-state temperatures is validated by experimental observations, as shown in Figure 1d. $\tau$ is generally found to be between 10 and a few hundreds of seconds for the great majority of experimental reports. As a first approximation, this value does not depend much on the material that composes the TH. Accordingly, this range of $\tau$ 260 values was observed for THs composed of AgNW networks ${ }^{[45]}$, CNT networks ${ }^{[27]}$, $\mathrm{TCO}^{[84]}$, conductive polymers $^{[50]}$, electrospun nanofibers ${ }^{[76,85]}$ or graphene ${ }^{[86]}$. For efficient THs, the $\tau$ 


\section{WILEY-VCH}

262 value mainly depends on the characteristics of the substrate: the thinner the substrate, the shorter 263 the characteristic time. For example, the steady state temperature was achieved within $10 \mathrm{~s}$ 264 when graphene films were deposited on a $50 \mu \mathrm{m}$ thick polyimide (PI) film, while it took $80 \mathrm{~s}$ 265 using $1 \mathrm{~mm}$ thick quartz as a substrate ${ }^{[86]}$. This is coherent with equation (4). For very small 266 substrate thicknesses $\tau$ can be very short too. Kim et al. demonstrated, as depicted in Figure 1e, that the use of single-walled carbon nanotubes (SWNTs) on a very thin polymeric substrate (12 $\mu \mathrm{m}$ thick) leads to a fast response: a temperature of $100{ }^{\circ} \mathrm{C}$ could be reached within $1 \mathrm{~s}$ with an input voltage of $7 \mathrm{~V}^{[61]}$. Ke et al. used the same idea and obtained similar results on Al-doped zinc oxide deposited on 25, 65 and 100 micron thick mica substrates ${ }^{[60]}$.

271 According to equation (2), the steady-state temperature elevation, $\Delta T_{s t a b}$, appears to be directly 272 proportional to the applied areal power density and, as a first approximation, does not drastically 273 depend on the TH type. Figure $1 f$ presents the areal power density dependence of the stabilized 274 temperatures measured for different $\mathrm{TH}$ technologies and demonstrates this trend. A linear 275 dependency is generally observed (data for $\mathrm{TH}$ based on $\mathrm{AgNW}^{[46]}, \mathrm{Ag} \mathrm{mesh}^{[82]}, \mathrm{CNT}^{[61]}$, PEDOT:OTf $f^{[50]}$ ) at least for temperatures lower than $100{ }^{\circ} \mathrm{C}$. The slope appears rather similar 277 for the different TH technologies. These slopes depend on the different thermal losses which 278 vary also according to the surface properties of the TH thin film and the substrate ${ }^{[60]}$. The 279 different thermal losses are convection, infra-red radiation and thermal conduction outside the 280 TH itself (through the TH substrate/holder or the electrical connecting wires). Several 281 investigations have tried to estimate the contribution of each thermal loss mechanism, which in 282 turn depends on the substrate, temperature and TH material used. Sorel et al. discussed their 283 dependence versus AgNW network density and showed that there is an increase in convection 284 losses for denser networks which is caused by a higher surface roughness and higher internal 285 area ${ }^{[43]}$. The thermal conductivity and thickness of the substrate play a key role for thermal conduction losses. In general, radiative losses are negligible compared to conductive and/or 


\section{WILEY-VCH}

288 losses can contribute significantly, and the linear approximation is not valid anymore. The

289 linear dependence between the steady-state temperature and the areal power density is therefore not valid anymore. For instance, Ji et al. reported the fabrication of highly-stable THs based on ultra-long copper nanofiber networks that can be heated up to $800{ }^{\circ} \mathrm{C}^{[87]}$. In this case, the steady-

state temperature versus heat power does not show a linear dependence in the entire temperature range (as reported in the supplementary information of $\mathrm{Ji}$ et al). ${ }^{[87]}$ Interestingly, comparing the same $\mathrm{TH}$ in air and in vacuum conditions allows us to disentangle convective losses from conduction and radiation losses. Lagrange et al. performed similar experiments on AgNW networks deposited on glass ${ }^{[46]}$ : in air and in vacuum the temperature elevations increase linearly with the areal power density, but with different slopes. The difference between these two slopes (23.8 and $28.1 \mathrm{~W} \cdot \mathrm{m}^{-2} \cdot \mathrm{K}^{-1}$ in vacuum and in air, respectively) allows us to deduce convective thermal losses: $h_{\text {convective }}$ could be estimated as $4.3 \mathrm{~W} \cdot \mathrm{m}^{-2} \cdot \mathrm{K}^{-1}{ }^{[46]}$ Another interesting discussion was also reported by Kang et al. regarding the influence of the CNT film density on the different types of heat-transfer losses. ${ }^{[88]}$ This can be understood through the heat-transfer losses that are directly related to the porous morphology of the CNT network. ${ }^{[88]}$

One can already classify THs into two different categories: on one side, the homogeneous layers

304 like transparent conductive oxides (TCO), graphene, multilayers (composed of a thin metal surrounded by oxide layers) or conductive polymer films; on the other side, networks composed of carbon nanotubes, metallic nanowires, meshes and fibers. While the first category is supposed to heat homogeneously the whole TH surface, the second category can induce local 308 heating due to its non-homogeneous nature, if heat does not diffuse along both the active 309 material and/or the substrate quickly enough ${ }^{[54]}$. These hotspots can induce local degradation within the TH and are therefore an issue to be dealt with. Das et al. investigated scaling in self-

311 heated percolating networks and concluded that hotspot clustering appears to be a mechanism 312 analogous to crystallization physics ${ }^{[89]}$. The potential presence of hotspots will be discussed in 313 this review, since their presence can lead to TH instability. In brief, the thermal stability of the 


\section{WILEY-VCH}

314 TH can be limited to the TH itself (e.g. degradation of the conductive polymer or morphological

315 instability of silver nanowires ${ }^{[90]}$ ) or to the substrate as well. Specifically, for polymeric

316 substrates or low-quality glass substrates, heating can lead to local melting of the polymer or

317 cracking of the glass substrate. For instance, Maize et al. recently used the thermo-reflectance

318 technique to observe "super-Joule" heating hotspots in graphene and AgNW networks ${ }^{[91]}$, while

319 the latter would have appeared homogeneous using a macroscopic infrared technique. This

320 emphasizes the importance of spatial resolution in temperature observations and this point,

321 among others, is discussed in the following section devoted to the experimental methods used

322 to investigate the main properties of THs. It is worth mentioning that industrial needs require

323 that a good heater should exhibit uniform thermal distribution over the heating area and achieve

324 the target temperature often with a low voltage (i.e. below $12 \mathrm{~V}$ ).

\subsection{Experimental methods to investigate the main properties of THs}

327 This section aims at introducing the main properties of THs and the experimental methods used 328 to investigate them. Generally speaking, a TH is considered efficient if it shows low electrical 329 resistance, high optical transmittance at least in the visible range, a controllable heating rate, 330 good stability and a low haze factor. We will first focus on electrical resistance and optical transparency. Then, we will present the methods to investigate the basic operating modes of a

$332 \mathrm{TH}$, the heating performances, which are particularly studied by temperature measurements at 333 different length scales. This enables us to measure characteristic features like the heating rate 334 and the relationship between temperature and areal heat power density. Regarding the 335 temperature, there are several ways to measure it through direct contact or contactless means, 336 and with different spatial resolutions, as illustrated below. Since the market appeals prompt 337 research for flexible (or even stretchable) $\mathrm{TH}$, the methods to investigate their mechanical 338 properties are also discussed. Thermal, electrical, humidity and/or mechanical stresses are also 


\section{WILEY-VCH}

reviewed. Finally, other TH features like haze factor, adhesion and roughness are briefly described, as well as their experimental investigation.

Electrical properties. Electrical resistance is a key property of THs, and is evaluated using several experimental methods. The sheet resistance $R_{s h}$ (in $\Omega / \mathrm{sq}$ ) is traditionally used to characterize uniform thin films, since it provides a direct and local measurement of electrical properties, independent of specimen size. The four-point probe method allows a precise measurement of $R_{s h}$ since it eliminates the contributions of electrical wiring and contacts to the global electrical resistance. ${ }^{[92]}$ This method is generally performed for emerging Transparent Conductive Materials (TCMs) such as thin films or metallic nanowire networks, despite being a direct contact method which can induce local damage. This electrical measurement is very valuable for estimating the electrical homogeneity of a sample by measuring its electrical performance in many different areas. As a first approximation and for a homogeneous layer, $R_{s h}$ is equal to the ratio between electrical resistivity and layer thickness, the latter being often measured by an electronic microscope, a profilometer, Atomic Force Microscopy (AFM) or an ellipsometer. Basic two-point probes can also provide the electrical resistance on samples. In general, two parallel electrodes at opposite sides of the specimen are fabricated using silver inks or metallic layers deposited by evaporation or sputtering. This two-probe method is generally used to follow in-situ the electrical resistance measured during voltage or thermal ramps ${ }^{[46,90]}$. For a homogeneous square layer with good electrical conductivity, the 4-probe and 2-probe methods are expected to provide similar results. Another tool to investigate electrical homogeneity is the one-probe electrical mapping, which draws up a cartography of voltage distribution. ${ }^{[93]}$ In addition, terahertz spectroscopy has been used for non-contact measurement of the conductance of AgNW networks. ${ }^{[94]}$ 


\section{WILEY-VCH}

364 Optical properties. A critical feature of THs is their optical transparency. As shown in Figure

365 2a, TCOs like FTO exhibit a very good transparency in the visible spectrum (390 to $700 \mathrm{~nm}$ ).

366 However, their optical transmittances decreases dramatically in the NIR spectrum due to

367 plasmonic absorption (as described in section 3.1). Emerging TCMs such as metallic grids, or

368 metallic nanowire and CNT networks, allow the incident light to be transmitted through the

369 non-covered empty substrate spaces, and thus they remain transparent to a great extent in the whole VIS-NIR spectrum. The transmittance of an AgNW network is shown in Figure 2a: this network is still very transparent in the NIR region. In the case of THs, most applications are in the visible range, which explains why most reports present only visible light measurements. Total transmission can be measured precisely by UV-visible-NIR spectrophotometry with an 374 integrating sphere, as schematically depicted in Figure $2 \mathrm{~b}$. There are also small and handy 375 instruments like tint meters, which provide transparency values at a certain visible wavelength, usually $550 \mathrm{~nm}$ (light wavelength for maximum efficiency of human eyes). Generally speaking, the optical transparency is either reported in literature at this wavelength of $550 \mathrm{~nm}$ or averaged over a typical range of $370-700 \mathrm{~nm}$ (i.e. the visible range), but unfortunately it is not always reported whether authors refer to specular (i.e. direct) or total transmittance. The measurement 380 of diffuse transmission is of crucial importance. It is generally expressed through the haze 381 factor, defined as the ratio between the diffuse and total transmittances (Figure 2b). Figure 2c shows a typical example of a low and a highly diffuse/hazy FTO. The non-hazy FTO is the usual FTO layer deposited on a glass substrate, while the diffuse FTO was fabricated by 384 depositing oxide nanoparticles before FTO deposition ${ }^{[59,95]}$. The required haze factor values 385 depend drastically upon the desired application. For displays or window defrosting, low haze values are mandatory. For other applications, however, this is less stringent. Although it is an 387 important characteristic, TH haziness is not often reported, preventing the complete 388 benchmarking of TH technologies. In the case of AgNW networks, the haze factor is directly 


\section{WILEY-VCH}
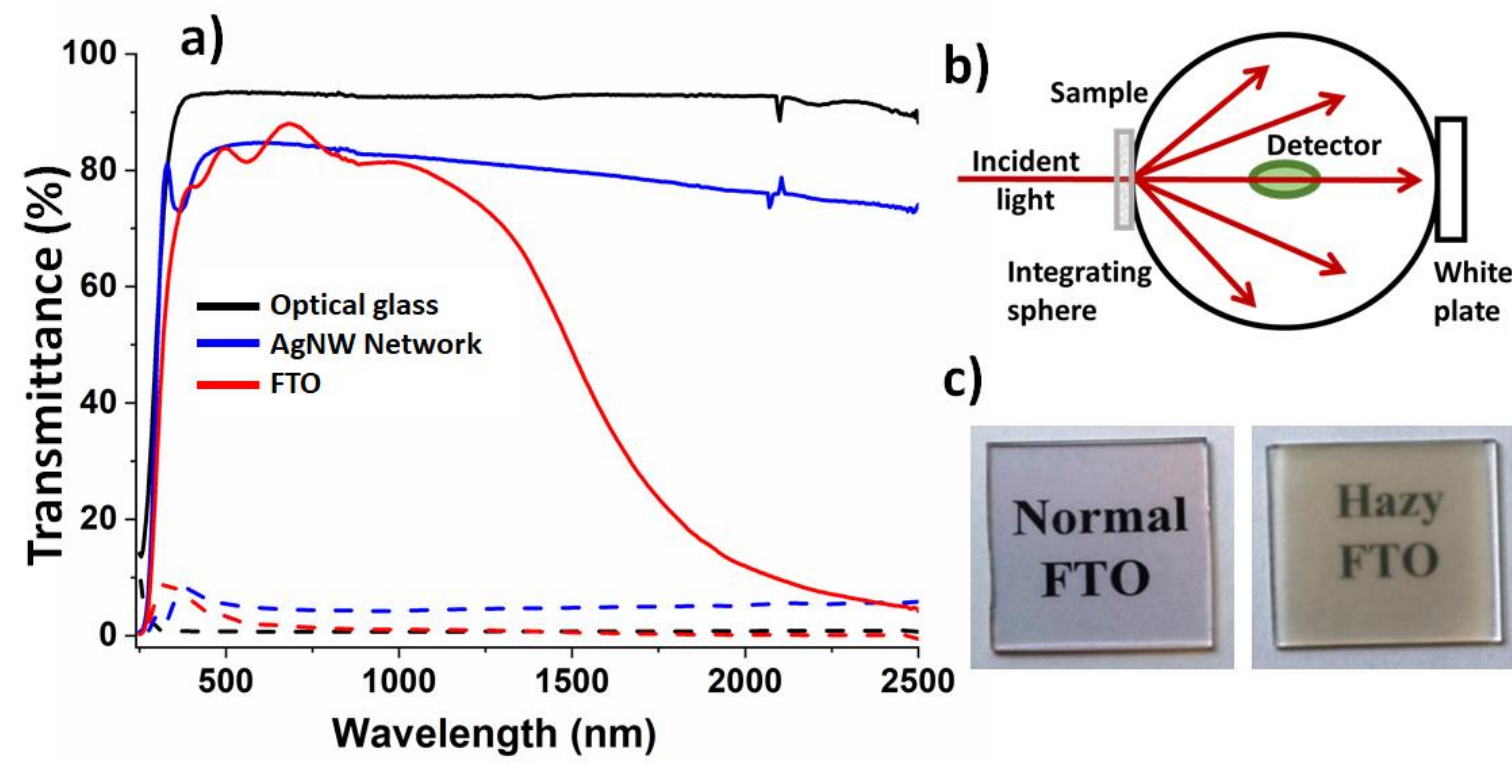

c)

Figure 2. Optical transmittance of transparent heaters (TH). a) Total and diffuse transmittance, shown respectively in continuous and dashed lines, for an AgNW network and an FTO layer deposited on bare optical glass. Both layers have a sheet resistance close to $20 \Omega /$ sq. Metallic nanowire networks are transparent in the NIR region. b) Schematic representation of the spectrophotometer with the integrating sphere. Total (diffuse) transmittance is measured with (without) the white plate to include (exclude) the specular transmittance. c) Pictures of non-hazy (left) and hazy (right) FTO layers on $25 \times 25 \mathrm{~mm}^{2}$ glass substrates.

Temperature measurement. The accurate measurement of temperature is essential for the fine the input voltage or other tuned parameters is expected with high temporal and spatial in the previous section 2.1, such as steady-state temperature, heating rate, operating voltage, heat power density, thermal resistance or conductivity, heat transfer coefficient, etc. A common choice for measuring the temperature is the use of a thermocouple, directly connected to the studied sample or device. Thermocouples are cheap and cover a wide range of temperatures. There are several suitable types of thermocouples depending on applications and the temperature ranges. ${ }^{[98]}$ The most reported thermocouple in the case of THs is the type $\mathrm{K}$ 


\section{WILEY-VCH}

411 thermocouple. Another way to measure the temperature by direct contact is the use of resistance temperature detectors (RTD) which are based on the increasing electrical resistance of

413 conductors with increasing temperatures. The platinum RTD in particular displays a superior 414 stability and excellent repeatability, but is more expensive than common thermocouples, and 415 has a narrower range of temperature measurement. ${ }^{[99]}$ Although these types of thermometers 416 are accurate and easy to use, some studies on THs require a precise mapping of the temperature 417 distribution that cannot be performed properly with the sensors described above. Thermal 418 imaging IR cameras detecting radiations in the mid or long-IR range of the electromagnetic spectrum are appropriate. They have become an indispensable experimental tool for specific temperature measurements. The spatial distribution of temperature is an important advantage

421 of IR imaging since it allows the investigation of heating homogeneity, reveals defects that are 422 optically invisible, and moreover it provides the temperature distribution globally in a device and its environment. Radiative temperature measuring is a non-contact method that can be much more convenient when it is hard or impossible to contact with the studied TH. However, IR imaging gives access only to the surface temperature, which is a clear disadvantage, but this does not appear to be a problem for THs since the active films are very thin. Some key parameters for an IR camera are the temperature range and the thermal sensitivity, the spatial resolution and the frame rate. The pixel pitch of these cameras has decreased to 10 microns, and recent studies suggest that it can be lowered down to 5 microns. ${ }^{[100]}$ Other critical issues of IR imaging are emissivity and reflection. In modern IR cameras these parameters can generally

431 be set by the operator. Almost all of the reported studies for THs use IR imaging. Emissivity is 432 rarely investigated experimentally ${ }^{[101,102]}$; that being said, Kim et al. showed that for AgNWcarbon hybrid THs, the emissivity of the tested film heater decreased linearly with the AgNW 434 content $^{[102]}$. This information is useful for the quantitative assessment of thermal losses. Finally, 435 another method with higher sensitivity and a submicron spatial resolution is thermoreflectance 436 (TR). It detects temperature changes near the surface using the relationship between optical 


\section{WILEY-VCH}

437 reflectance and temperature. ${ }^{[103]}$ It is used for very specific temperature studies, and not to

438 record the temperature in large areas since it is difficult to define an accurate absolute 439 temperature., In the case of AgNW networks for example, TR imaging can record hotspots of 440 individual nanowire junctions in the $300 \times 300$ nanometer region size. ${ }^{[1]}$ In another case a

441 picosecond TR technique was used to study the thermal diffusivity of 30 to $70 \mathrm{~nm}$ thick ITO 442 thin films. ${ }^{[104]}$ Figure 3 presents examples of IR and TR imaging with the corresponding size 443 scales. Figure 3a represents the temperature profile of AgNW-rGO (reduced graphene oxide), 444 measured by an IR camera with a low pixel resolution of $250 \mu \mathrm{m}$ x $250 \mu \mathrm{m}$. This roughly 445 corresponds to an area with more than one hundred nanowires. ${ }^{[54]}$ A comparable technique used 446 to reveal defects in microelectronic devices is lock-in thermography (LiT). ${ }^{[105]}$ It can provide 447 an IR emission intensity and a heat timing map ${ }^{[106]}$ but does not include any thermal calibration 448 or numerical scale. However, as shown in Figure $3 \mathrm{~b}$, it appears very useful to visualize the 449 electrical and thermal distribution in the case of MNW networks. It enables us to investigate 450 the activation of efficient percolating pathways and the detection of hot spots under electrical 451 stress. ${ }^{[107]}$ In the case of the IR camera shown in Figure $3 \mathrm{c}$, the higher resolution of $1.6 \mu \mathrm{m}$ 452 helps identify the temperature of each channel of the metal crack template. ${ }^{[108]}$ Finally, 453 Figure $3 \mathrm{~d}$ shows how advantageous TR can be to perform temperature studies, for example to 454 image hot spots and nanowire junctions. ${ }^{[1]}$ 


\section{WILEY-VCH}
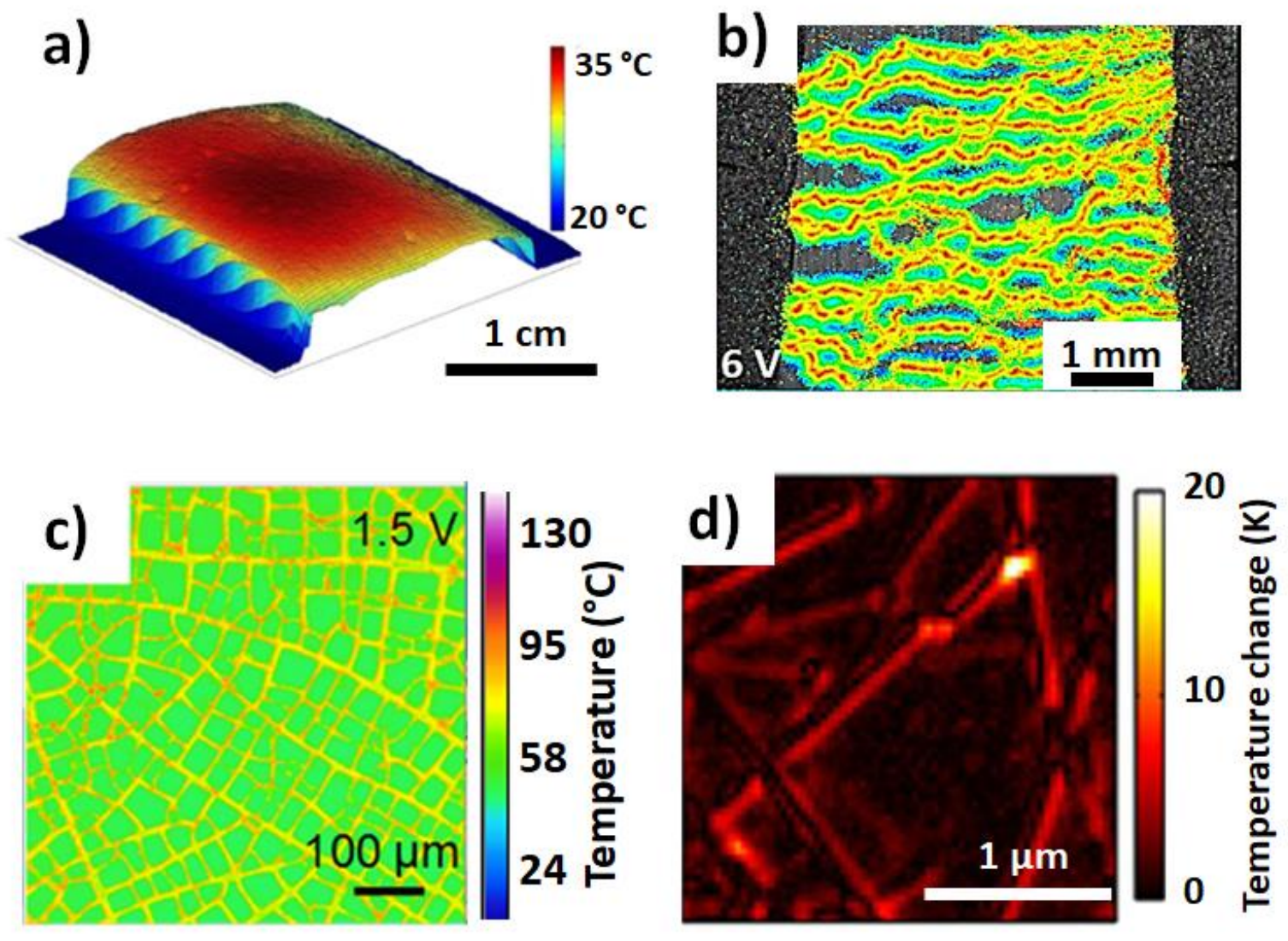

Figure 3. Thermal imaging methods for characterizing THs at different length scales. a) 3D temperature profile measured using an IR camera with $250 \mu \mathrm{m}$ x $250 \mu \mathrm{m}$ pixels, for AgNW-rGO after applying an electrical current of $20 \mathrm{~mA}$ for $60 \mathrm{~s}$. Reproduced with permission. ${ }^{[54]}$ Copyright 2017, IOP Publishing. b) LiT image of an AgNW network. The efficient percolating pathways can be detected in brighter colors with a camera of $640 \times 512$ pixels resolution with $10 \mathrm{~Hz}$ "ON-OFF" voltage cycles of 6V. Reproduced with permission. ${ }^{[107]}$ Copyright 2016, American Chemical Society. c) Thermal map of a metal mesh network fabricated from a crack template under $1.5 \mathrm{~V}$, with IR imaging with $1.6 \mu \mathrm{m}$ resolution. Reproduced with permission. ${ }^{[108]}$ Copyright 2017, American Chemical Society.d) Enlarged region of a nanowire network channel, showing representative hotspots using thermoreflectance (TR) imaging. Reproduced with permission. ${ }^{[91]}$ Copyright 2015, American Chemical Society.

The different types of temperature measurements are carried out and presented as a function of

the electrical properties, since the Joule effect is responsible for the heating. As mentioned previously, the steady-state temperature $\left(T_{\text {steady }}\right)$ is an important feature for THs and can be studied experimentally by applying a constant voltage or areal power density. The time necessary to reach the steady-state temperature is also measured, and the heating rate is usually

471 calculated and reported with the corresponding value of the applied voltage. Plotting $T_{\text {steady }}$ versus the input power density values shows a linear relationship, with the slope coefficient representing the thermal resistance per unit of area. Another typical experiment related to 


\section{WILEY-VCH}

474 performance measurements of THs is the cyclability or "ON-OFF" test, which consists of 475 switching between the on and off states of the applied bias for a large number of operating cycles. $^{[102,109]}$

Mechanical properties. The first application of THs was during World War II, with the use of stiff tin oxide to defrost the cockpit windshields of aircrafts to allow them to fly at higher altitudes. ${ }^{[2]}$ These past years, the development of nanotechnology and advances in flexible electronics have given rise to a huge variety of applications that require excellent stability under mechanical stresses (flexibility and even stretchability). As explained hereinafter, TCOs cannot be integrated into very flexible devices, despite their excellent electrical and optical properties. The high temperature deposition process makes it rather impossible to use flexible and transparent polymers as substrates, because these do not have high thermal resistance. ${ }^{[110]}$ When they are deposited on flexible substrates, the electrical performance of TCOs is often limited due to deformations that appear on these thin films. On the other hand, the flexibility of emerging materials such as CNTs, metallic grids, MNWs and conductive polymers is one of the main reasons why their study is constantly increasing. The measurement of electrical resistance over thousands of bending cycles is a standard experiment reported in the literature. In general, the bending radius (usually of several $\mathrm{mm}$ ) is provided. This value is important since it indicates to what extent the TH can be bent, sometimes crumpled. For THs, electrical conductivity must not be altered by mechanical stresses, and the thermal performance should remain stable. In order to estimate the mechanical behavior of THs, the temperature is usually monitored simultaneously to bending. IR imaging, as a contactless method, is particularly convenient for the direct evaluation of performances. Figure 4a presents typical examples of bending tests while Figure $4 \mathrm{~b}$ exhibits stretching tests with concomitant temperature recording by IR camera. Twisting tests, as shown in Figure 4c, are also sometimes reported, depending on the specific application needs. High flexibility has become a common target for emerging 


\section{WILEY-VCH}

499 TCMs. Interest has also recently been focused on stretchability, which is intrinsically more

500 complicated. The loss of electrical conductivity can be either total, low or negligible depending

501 on several parameters such as the amount of conductive material or the choice of material itself.

502 Ongoing investigations on stretchable THs with an association of different materials (leading

503 to nanocomposites or hybrid materials, see section 3.5 ) lead to promising features. ${ }^{[22,70,111]}$

504 Finally, the incorporation of TCMs into elastic substrates, and the adhesion to various types of

505 substrates is another issue concerning mechanical properties which could influence the

506 performance of $\mathrm{TH}$ applications. Embedded structures can be a promising strategy to tackle

507 adhesion issues. ${ }^{[81]}$ Measuring performance stability after repeating tape tests ${ }^{[47,112,113]}$ and

508 microscopic observations of the adhesion between the layers are useful techniques to determine

509 the key drivers that should be studied in depth. 


\section{WILEY-VCH}
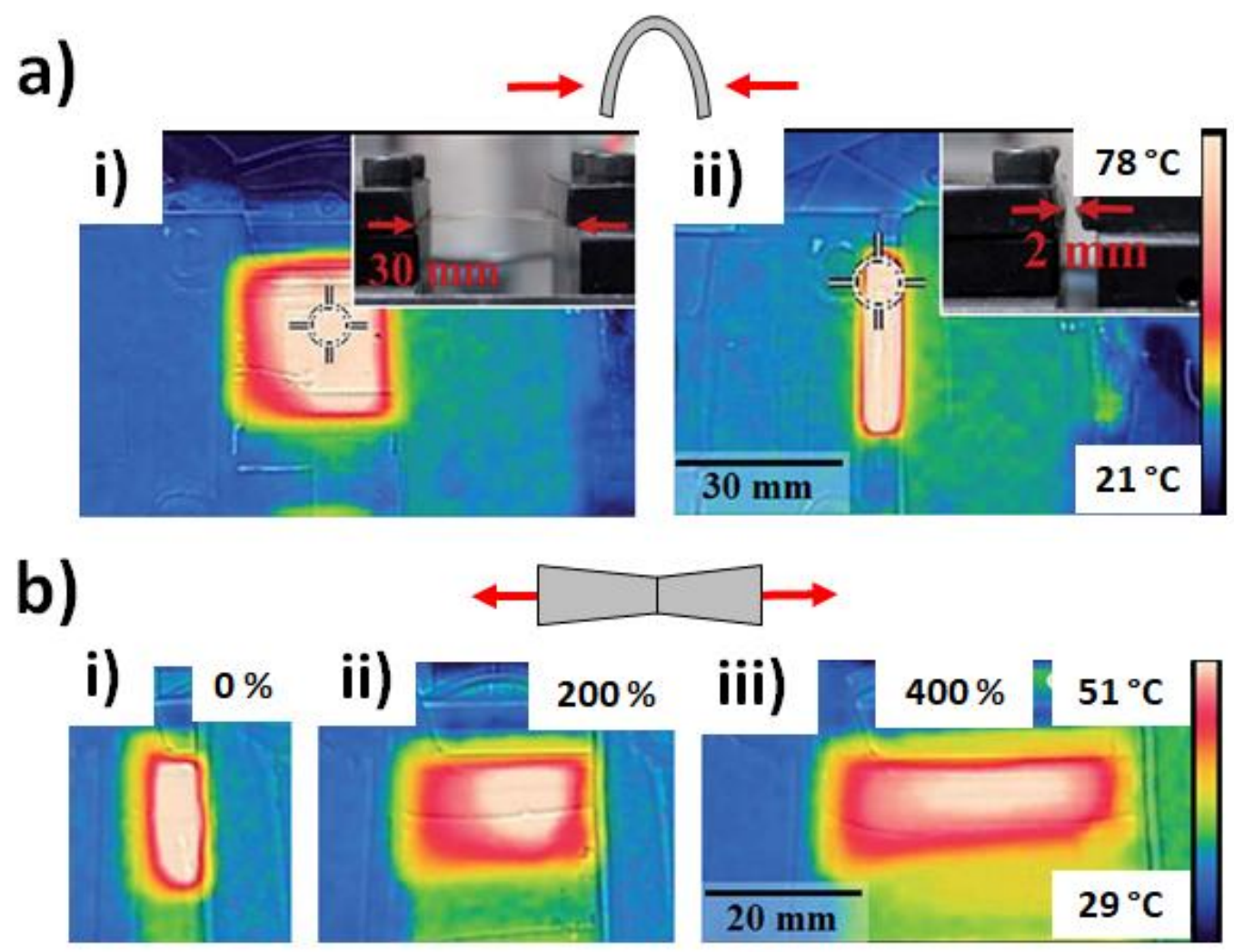

c)
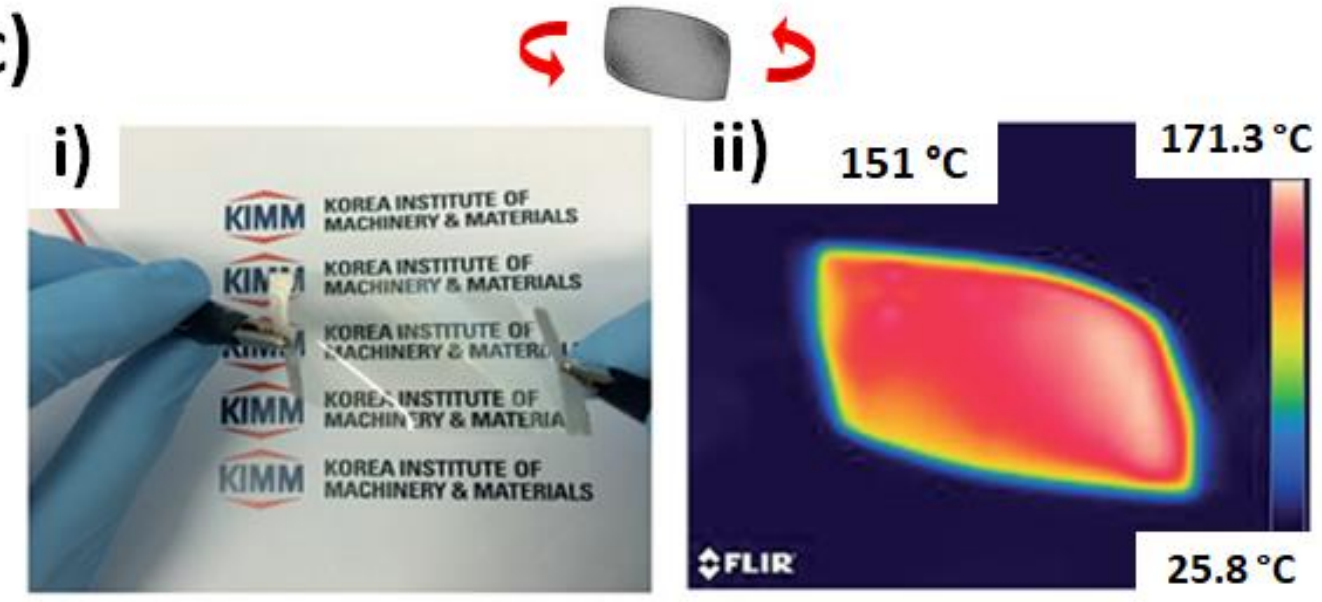

Figure 4. Infrared observations of THs when submitted to different mechanical stress modes. a) Photographs and IR images of an AgNW film during bending tests at i) the initial conformation and ii) the final conformation. The AgNW film $(R s h=15 \Omega / \mathrm{sq}, T r=95 \%)$ was supplied with a voltage of $5 \mathrm{~V}$. b) During the stretching experiment at: i) the initial state $(\mathrm{L}=10 \mathrm{~mm})$, ii) the intermediate stages of stretching with the strain values in percentage shown in the insets, and iii) the final state $(\mathrm{L}=50 \mathrm{~mm})$. The substrate was a stretchable eco-flex. a,b) Reproduced with permission. ${ }^{[114]}$. Copyright 2017, Royal Society of Chemistry. c) Mechanical flexibility and stability of a CuNi TH on a polymer substrate: i) picture and ii) IR image of the heater at $9 \mathrm{~V}$ bias under twisting conditions. Reproduced with permission. ${ }^{[15]}$ Copyright 2017, Royal Society of Chemistry.

Stability. Electrical, thermal, mechanical and chemical stabilities are key parameters for TH

performance. Firstly, it is necessary to verify that electrical conductivity and heat emitted 


\section{WILEY-VCH}

523 remain stable in the long run when THs undergo electrical stresses. Several tests can be

524 performed, like a voltage ramp and/or plateau cycles with resistance and temperature

525 recordings. The "ON-OFF" test described above is another typical stability experiment that is

526 performed in most TH studies. The application of a constant voltage/power for hours in order

527 to check if the temperature remains stable ${ }^{[116]}$ is also usually carried out. In other cases,

528 constantly increasing voltage ramps are applied until the breakdown of the heating

529 performance, followed by the application of lower voltages to investigate potential

530 reversibility. ${ }^{[46]}$ These experiments have proven that the encapsulation of heating materials with

531 protective coatings is an excellent choice to improve stabilization. Thermal stability is crucial

532 for THs and it is linked to the potential degradation of active materials or surrounding materials

533 due to high operating temperatures and fast heating/cooling rates. A way to study this is to

534 measure electrical resistance under thermal stress (thermal ramps or plateaus) without applying

535 any electrical power (except a very small current for the resistance measurement). It is also

536 important to investigate the intrinsic heat stability of the substrates used to fabricate a TH,

537 especially in the case of flexible polymers, which are known to limit the stability of flexible

538 THs. Regarding chemical oxidation and ageing, typical measurements are carried out in

539 environmental chambers and involve the simultaneous exposure to controlled humidity and

540 temperature, combined with light irradiation ${ }^{[48,117]}$. Generally, the relative humidity chosen is

541 over $80 \%$, and the temperature between $45^{\circ} \mathrm{C}$ and $90^{\circ} \mathrm{C}$, which covers most of the harshest use

542 conditions. ${ }^{[70,93,118-121]}$ Study durations varies strongly, and usually depends on the anticipated

543 stability of the TH and on the expected lifetime of its components. For example, stability studies

544 for copper nanowire $(\mathrm{CuNW}) / \mathrm{NPs}$ usually last minutes ${ }^{[120]}$ or hours ${ }^{[118,119]}$, while for AgNW

545 the duration of the study can be days ${ }^{[70]}$ or months ${ }^{[121]}$. Of note, there are some large

546 discrepancies in terms of MNW network stability from one research group to another, probably

547 originating from differences in MNW growth conditions and purification processes, leading to

548 different surface chemistries, and consequently to more or less stable MNW networks. 


\section{WILEY-VCH}

\section{The investigated materials technologies for transparent heaters}

551 Several material technologies for TH applications have been thoroughly investigated. The very

552 first type of thin layers were TCOs, which have been investigated for several decades ${ }^{[2,9,14,17,59]}$.

553 These thin films of metal oxide have been studied for several applications, including transparent

554 electrodes for solar cells or touch screens, and have already been widely integrated in industrial 555 devices. ${ }^{[7,10]}$ For THs, like for transparent electrodes, industrial needs have prompted research 556 into other materials ${ }^{[21]}$. There are several reasons for this. TCOs, for instance, are ceramic and 557 therefore do not usually withstand any mechanical stress; this makes them incompatible with 558 flexible applications. Moreover, lowering the cost is a strong argument against the vacuum559 process and/or the use of indium in the classical indium tin oxide (ITO), due to the scarcity of 560 indium. Likewise, the search for properties that are better adapted to industrial requirements 561 necessitates the exploration of other materials. The goal of this part 3 is to present the main 562 materials categories and their related properties when used for THs. Each type of materials will 563 then be discussed in more detail below, starting with metallic oxide-based materials, then 564 carbon-based nanomaterials (graphene and CNTs), metallic-based materials, conductive 565 polymers and finally nanocomposites or hybrid materials. In this review we consider that 566 nanocomposites are materials for which the nanostructures are within a continuous phase such 567 as a matrix, while hybrid nanomaterials refer to combination of materials of different natures 568 such as organic-inorganic.

\subsection{Metallic oxide-based materials}

571 Among all the different types of TCMs, TCOs were the first to be developed and studied. They

572 have been used industrially for a long time and in many different applications. ITO thin films, 573 in particular, have been employed for the production of THs to defrost aircraft windshields. The 574 basic properties of TCOs have been extensively described in previous works, and we refer the 


\section{WILEY-VCH}

d)
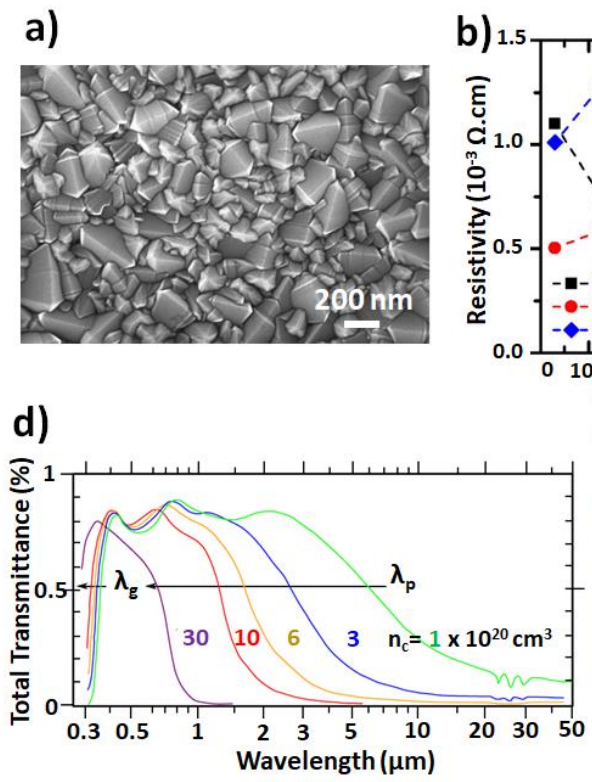

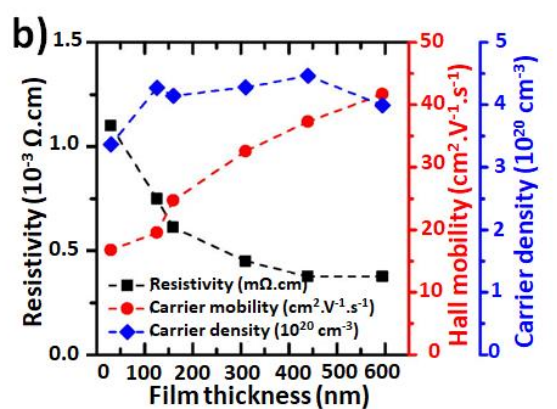

e)

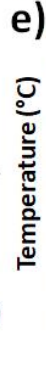

reader to those for additional details ${ }^{[9,122,123]}$. Of note, the morphology and structure of TCO thin films play a key role in their conductivity. These thin films usually exhibit polycrystalline structures, as shown in Figure 5a, with crystal sizes that depend on the deposition method used and other parameters like film thickness. The latter can easily reach hundreds of nanometers. ${ }^{[14]}$ Twins are often observed (for FTO for instance), and as in the case of grain boundaries, they are detrimental to charge mobility ${ }^{[14,59,124]}$. The fact that grains often become bigger as the film thickness increases has a direct impact on the conductivity of the layers, since the number of grain boundaries and twins decreases (Figure 5b). Another important aspect of TCOs is the fact that transparency in the IR region of the spectrum falls rapidly with film thickness or with carrier concentration due to plasmonic absorption as shown, respectively, in Figure $5 \mathrm{c}$ and 5d. ${ }^{[20]}$ For TH applications where IR transparency is important, e.g. night vision applications for glasses and binoculars, the TCO technology is therefore not perfectly adapted compared to other TH technologies.

Figure 5. Transparent conductive oxides (TCO) used as THs. a) SEM image of a typical $400 \mathrm{~nm}$ thick FTO layer showing the grain-boundary structure of the thin polycrystalline film. b) Main electrical characteristics of FTO layers vs film thickness: electrical resistivity, carrier mobility and carrier density. Reproduced with permission. ${ }^{[14]}$ Copyright 2013, AIP Publishing. c) Total optical transmittance for different FTO film thicknesses: the thicker the film, the lower the transmittance, specifically in the near infra-red region due to plasmonic absorption. d) Computed spectral normal transmittance of ITO. The values correspond to electron density. Reproduced with permission. ${ }^{[125]}$ Copyright 1986, AIP 


\section{WILEY-VCH}

596 Publishing. e) Time dependence of temperature when a $12 \mathrm{~V}$ voltage is applied to a TH composed of either an AZO layer (black), a single FTO layer (green), an AZO/FTO (blue) or a FTO/AZO (red) double-layer film on glass which shows different electrical properties. This clearly illustrates the strong influence of the TH electrical properties on the heating performance. Reproduced with permission. ${ }^{[84]}$ Copyright 2014 John Wiley and Sons. f) Measured temperature vs time for a $100 \mathrm{~nm}$-thick AZO-based $\mathrm{TH}$, deposited on mica substrates of different thicknesses, illustrating the role played by the thermal inertia of the substrate in terms of response time. Reproduced with permission. ${ }^{[60]}$ Copyright 2018, AIP Publishing.

Despite TCOs being the oldest studied TCMs, there are surprisingly few reports in the literature dealing with the application of TCOs as THs. The studies reported so far involve ZnO-based materials, Ga-doped $\mathrm{ZnO}(\mathrm{GZO})^{[126-128]}$ and Al-doped $\mathrm{ZnO}(\mathrm{AZO})^{[60,84,127,129]}, \mathrm{FTO}^{[84]}$ and ITO $^{[130-133]}$. Indeed, TCOs have been mainly investigated in the framework of industrial research and development, which is generally not associated with scientific publications.

In industry, ITO is routinely deposited by sputtering, a vacuum method that is generally associated with higher costs compared with solution process technologies. Im et al. explored

612 the utilization of ITO nanoparticle (NP) solutions to process ITO thin films ${ }^{[130]}$ as an alternative

613 to vacuum processing. The films were fabricated by spin-coating the ITO NP solution on a glass

614 substrate, followed by annealing up to $400{ }^{\circ} \mathrm{C}$. The main effect of the annealing step is to sinter

615 the particles. Using these films, a temperature of $163^{\circ} \mathrm{C}$ was achieved for a bias voltage of 20

$616 \mathrm{~V}$, and dry-ice was used to demonstrate the defrosting capabilities of the TH. ${ }^{[130]}$ More recently,

617 Kim et al. used the same approach. However, by increasing the annealing temperature of the 618 deposited ITO NP and by using different atmospheres during the annealing step, they 619 demonstrated that the oxygen vacancies in the film can be controlled ${ }^{[131]}$. In this case, 620 temperatures up to $215^{\circ} \mathrm{C}$ were reached for a $15 \mathrm{~V}$ bias, with a thermal efficiency of 162.9 $621{ }^{\circ} \mathrm{C} /\left(\mathrm{W} / \mathrm{cm}^{2}\right)$ being obtained for optimized samples. In another study, Yang et al. demonstrated 622 that by adding organic additives such as polymethyl methacrylate and terpineol to the ITO NP 623 solution, the obtained heaters are more efficient and homogeneous. The authors made micro624 heaters using this approach, and could reach temperatures of $445{ }^{\circ} \mathrm{C}$ for a bias voltage of only 


\section{WILEY-VCH}

626 Kim et al. explored the use of bilayers by combining AZO and FTO deposited by sputtering

627 and Chemical Vapor Deposition (CVD), respectively. ${ }^{[84]}$ They observed that both conductivity

628 and transparency improved for bilayers with respect to single layers of the same thickness, as

629 demonstrated in Figure 5e. ${ }^{[84]}$ This is attributed to the higher overall particle size in the film,

630 which affects both mobility and transparency. The best results, reported in Figure 5e, were

631 obtained for FTO/AZO bilayers for which temperatures above $70^{\circ} \mathrm{C}$ were obtained for a bias

632 voltage of $12 \mathrm{~V} \cdot{ }^{[84]}$ Roul et al. studied rigid and flexible AZO-based TCOs by depositing AZO

633 on glass and polyethylene terephthalate (PET) substrates by magnetron sputtering. ${ }^{[129]}$ The

634 films deposited on glass reached temperatures of approximately $100^{\circ} \mathrm{C}$ for a $12 \mathrm{~V}$ bias for the

635 samples deposited at $200{ }^{\circ} \mathrm{C}$. Flexible heaters made on PET reached $50^{\circ} \mathrm{C}$ for bias voltages of

$6368 \mathrm{~V}$. Nevertheless, stability results are only shown for AZO/glass samples, and no study of the

637 effect of bending on the properties of the flexible $\mathrm{TH}$ is reported. It is also worth mentioning

638 that $\mathrm{ZnO}$-based TCOs may not be the best candidates for high temperature applications, since

$639 \mathrm{ZnO}$ tends to absorb oxygen species in the grain boundaries, which has a detrimental effect on

640 mobility, as explained and modelled recently. ${ }^{[19]}$ It has been shown that the absorption of

641 oxygen species increases rapidly with temperature ${ }^{[134]}$, thus most likely limiting the applications

642 of THs based on AZO.

643 Ke et al. showed that despite the ceramic nature of ITO, flexible THs can be obtained by using

644 mica substrates as thin as $15 \mu \mathrm{m}$, thanks to their layered structure. ${ }^{[133]}$ ITO layers of up to 500

$645 \mathrm{~nm}$ were deposited by pulsed laser deposition (PLD) at room temperature and annealed at up to

$646500^{\circ} \mathrm{C}$. Flexible THs were fabricated, exhibiting fast ramping. Temperatures of up to $438^{\circ} \mathrm{C}$

647 were reached in less than $15 \mathrm{~s}$ at a bias voltage of $19 \mathrm{~V}$. The same approach was used by the

648 same authors to make flexible THs based on AZO/mica. ${ }^{[60]}$ Figure $5 f$ shows the measured

649 temperature versus time for a $100 \mathrm{~nm}$-thick AZO-based TH deposited on mica substrates of

650 different thicknesses: this illustrates the role played by the thermal inertia of the substrate, ${ }^{[60]}$ in

651 agreement with equation (4). Record heating rates of $200^{\circ} \mathrm{C} / \mathrm{s}$ were obtained in this case. The 


\section{WILEY-VCH}

652 occurrence of van der Waals epitaxy between the mica substrate and the grown AZO film

653 reasonably explained this superior performance.

654 Gallium has also traditionally been used to dope $\mathrm{ZnO}$. Jayathilake et al. deposited $\mathrm{ZnO}$ co655 doped with $\mathrm{Al}$ and Ga (AGZO) by aerosol-assisted chemical transport, which showed a lower 656 conductivity than AZO or GZO films ${ }^{[127]}$. The films were prepared by dispersing previously 657 prepared AGZO powders (by microwave synthesis) in a methanol solution containing formic 658 acid and cellulose. Then aerosol generated from this suspension was carried by $\mathrm{N}_{2}$ gas to a glass 659 substrate at $400{ }^{\circ} \mathrm{C}$. Temperatures above $132{ }^{\circ} \mathrm{C}$ were achieved in 10 min for bias voltages of

$66018 \mathrm{~V}$. Older reports on physically deposited GZO films were performed by Kim et al. ${ }^{[128]}$ and 661 Ahn et al.. ${ }^{[126]}$ In the first case, the films were deposited by rf-magnetron sputtering on glass 662 substrates ${ }^{[128]}$ and reached $90{ }^{\circ} \mathrm{C}$ after $22 \mathrm{~s}$ for a bias voltage of $48 \mathrm{~V}$. In the second case, PLD 663 was used to deposit the films on glass substrates, and reached over $88{ }^{\circ} \mathrm{C}$ after $48 \mathrm{~s}$ for a $12 \mathrm{~V}$ 664 bias. ${ }^{[126]}$

665 Thus, while TCOs, and in particular ITO, are the most prominent transparent materials today, 666 with a broad range of industrial applications, fundamental studies involving promising 667 approaches are still being carried out in order to push the limits of these materials in terms of 668 performance and mechanical flexibility. These studies are framed in a wider research that 669 involves the exploration of competing materials, such as carbon-based nanomaterials, which 670 are described in the next section.

\section{$672 \quad 3.2$ Carbon-based nanomaterials (CNTs \& graphene)}

673 Carbon-based thin film heaters were first described in 2007. ${ }^{[27]}$ Carbon fibers, carbon nanotubes

674 (CNTs) or graphene derivatives have been widely reported in the literature to address several 675 fields of applications including defogging, ${ }^{[135]}$ anti-icing, de-icing, ${ }^{[83,136,137]}$ wearable 676 electronics, ${ }^{[138,139]}$ thermochromic displays ${ }^{[140,141]}$ or thermomechanical sensors. ${ }^{[62]}$ 


\section{WILEY-VCH}

677 Most published studies report the use of carbon-based thin film heaters for the assessment of

678 non-transparent devices. Their high flexibility and stretchability are, without doubt, advantages

679 that make them very interesting for various type of heaters thanks to their remarkable

680 mechanical properties, while conversely they exhibit low transparency. ${ }^{[57]}$ Since this review

681 focuses on THs, only devices or active materials with good transparency are discussed

682 hereinafter.

683 Active parts of carbon-based THs can be processed either by dry or solution-based routes, the 684 latter being often more suitable for many supports (PEN, PET, PC, PI or cotton fabrics for example). CNT devices can be produced either through percolative networks implying printing 686 processes $^{[61]}$, or thanks to CNT forest (aligned CNTs, usually prepared by chemical vapor 687 deposition (CVD) / transfer protocol). For the first method, Figure 6a shows an SEM image of 688 a transparent and flexible single wall carbon nanotubes (SWCNT) heater prepared on a plastic 689 substrate, along the associated IR images at various applied voltages. ${ }^{[61]}$ Regarding graphene 690 and its derivatives, exfoliation of graphite or chemical reduction of GO were reported. Recently, 691 unusual and interesting approaches based on the use of carbon nanosheets from the 692 carbonization of polymers or from natural carbonaceous by-products of ethylene production 693 were published by Souri et al. ${ }^{[142]}$ and by Morris et al. ${ }^{[143]}$, respectively.

694 Both the deposition processes and the intrinsic properties of the material strongly impact the performances of carbon-based THs. For CNT networks, the trade-off between transparency and conductivity can also be controlled by nanotube types (e.g. single-walled vs multi-walled

697 MWCNT). SWCNTs generally exhibit high transparency and low haze, but rather low 698 conductivity due to the presence of poorly conductive semi-conducting CNTs and resistive 699 CNT-CNT junctions. ${ }^{[61,144]}$ MWCNT-based electrodes (100\% of metallic behavior tubes) are more conductive but less transparent due to their larger size, and exhibit a higher haze value. ${ }^{[145]}$

701 Figure $6 \mathrm{~b}$ exhibits optical and infrared pictures of graphene-based THs illustrating both 702 flexibility and uniformity. ${ }^{[57]}$ In the case of percolative networks of graphene flakes, each 


\section{WILEY-VCH}

703

704

705

706

707

708

709

710

711

712

713

714

715

716

717

718

719

720

graphene layer decreases the transparency by $\sim 2-3 \%$. However, devices made of a graphene monolayer obtained by dry deposition keep a very high level of transparency, but this technique is somewhat limited to small surfaces. Improvement is still required in terms of uniformity of highly conductive graphene layers and larger surface coverage. Due to the higher electrical contact resistances between the conductive objects of carbon-based networks (CNTs, Carbon flakes) compared to MNW networks, most carbon-based THs require denser systems to reach the same level of conductivity, at the expense of the transparency.

As input voltage and surface temperature are strongly correlated to sheet resistance, in most cases, carbon-based THs are addressed with high input voltages from 10 to $100 \mathrm{~V}$ for at most a few $\mathrm{cm}^{2}$ samples. Indeed, transmittances higher than $70 \%$ are achievable at several hundreds of $\Omega /$ sq. $^{[146]}$ On polyimide substrates, temperatures higher than $350^{\circ} \mathrm{C}$ have been reached. ${ }^{[142]}$ Considering that surface power densities of a few $\mathrm{kW} / \mathrm{m}^{2}$ are required for both thermal comfort and heating, the lifetime and stability of carbon-based materials are a true asset of carbon-based THs.

In the case of carbon-based THs, transparency is clearly impaired if one intends to reach low sheet resistance for high heating performance. New developments using carbon nanostructures hybridized with metallic nanowires could provide an interesting alternative since carbon-based materials can significantly enhance the stability of metallic nanowires (See section 3.5.1).

a)

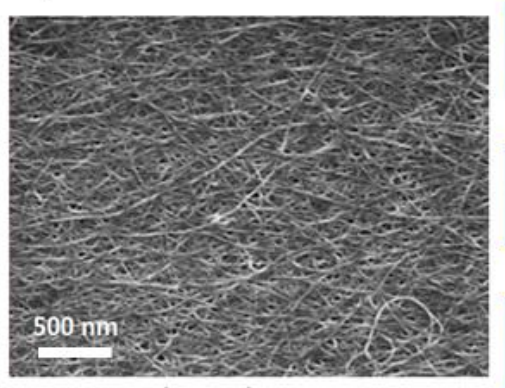

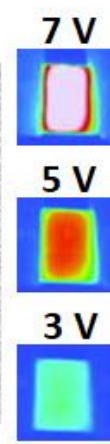

b)
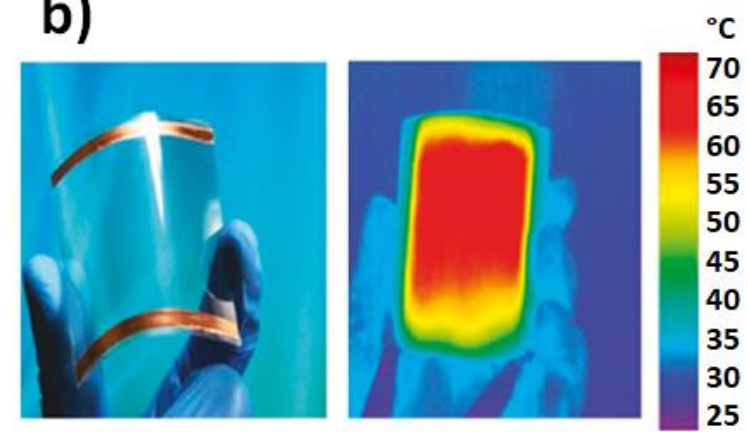

Figure 6. Performances of carbon-based transparent heaters on flexible substrates. a) SEM pictures and infrared pictures of SWCNT-based transparent electrodes when submitted to applied voltages of 3,5 or 7 volts. Reproduced with permission. ${ }^{[61]}$ Copyright 2017, AIP Publishing b) Highly flexible graphene- 


\section{WILEY-VCH}

based TH with optical and infrared pictures illustrating both flexibility and uniformity. Reproduced with permission. ${ }^{[57]}$ Copyright 2011, American Chemical Society.

\subsection{Metallic-based materials: metallic grids, metallic meshes, MNW random networks}

While TCOs have been largely studied for several decades to be used as transparent electrodes (TEs) or THs, other materials such as metallic-based nanostructures have been considered since the year 2000 approximately ${ }^{[21,147]}$. They present additional properties when compared with TCOs, since many of them can be deposited at low temperatures and exhibit good mechanical flexibility. Metals indeed appear attractive as potential TH active materials since they possess very high electrical and thermal conductivities. For instance, silver exhibits the highest conductivities among all materials at room temperature. However, ultrathin metallic films generally do not show an interesting trade-off between optical transparency and sheet resistance, due to both strong light absorption and electron scattering at surfaces or interfaces. As such, the only way to take advantage of metals for TH applications is to engineer metal nanostructures. This can provide interesting opportunities to manipulate photons and electrons in order to achieve electrical, optical and mechanical properties that are not reachable with TCOs. The main metallic patterns that can be efficiently used for TH production are metallic meshes, MNW networks and metallic grids. The difference between metallic grids and meshes in the literature is sometimes confusing. In this review we will consider that metallic meshes do not contain privileged directions in terms of conductive lines, while metallic grids are constituted by a periodic arrangement of metal lines, generally with a 3- or 4-fold symmetry. In this section, we will briefly examine the different investigated metallic-based materials, by describing their main features, properties and their potential integration into $\mathrm{TH}$ devices. Progresses toward low-cost TH fabrication, wire-wire junction resistance, physical properties, adhesion and stability will also briefly be discussed.

Metallic meshes can exhibit some interesting features as THs. As shown by Kiruthika et al. interconnected crackles can be obtained thanks to the spreading of low cost crackle wall paint 


\section{WILEY-VCH}

752 by using the drop coating technique. ${ }^{[82]}$ Interconnected $\mathrm{Ag}$ meshes can be fabricated by 753 depositing a thin metal layer on top of the obtained crackles and then removing the template.

754 Figure 7a shows a typical optical image of an Ag mesh fabricated on glass, while the inset 755 corresponds to the optical profiler image (the thickness is approximately $300 \mathrm{~nm}$ ). ${ }^{[82]}$ This type 756 of metallic mesh network exhibits interesting physical properties on large areas. Kiruthika et 757 al. reported a sheet resistance of $1 \Omega / \mathrm{sq}$ on an $18 \times 15 \mathrm{~cm}^{2}$ area, associated to an optical 758 transmittance of $77 \%{ }^{\left[{ }^{[82}\right]}$ Thanks to the low electrical resistance, the use of metallic meshes as 759 THs requires a rather low voltage: an $8.5 \mathrm{~V}$ bias was used to reach uniformly heated surfaces 760 of up to $170^{\circ} \mathrm{C}$. This efficient defrosting device successfully withstood an ultra-sonication test, 761 as well as many defrosting cycles. ${ }^{[82]}$ Metallic mesh networks have been the subject of many

762 studies lately, with various metallic species being evaluated, like copper, ${ }^{[148]}$ silver, ${ }^{[149,150]}$ 763 nickel, ${ }^{[151]}$ cupronickel, ${ }^{[115]}$ gold $^{[152]}$ and platinum. ${ }^{[72]}$ 


\section{WILEY-VCH}
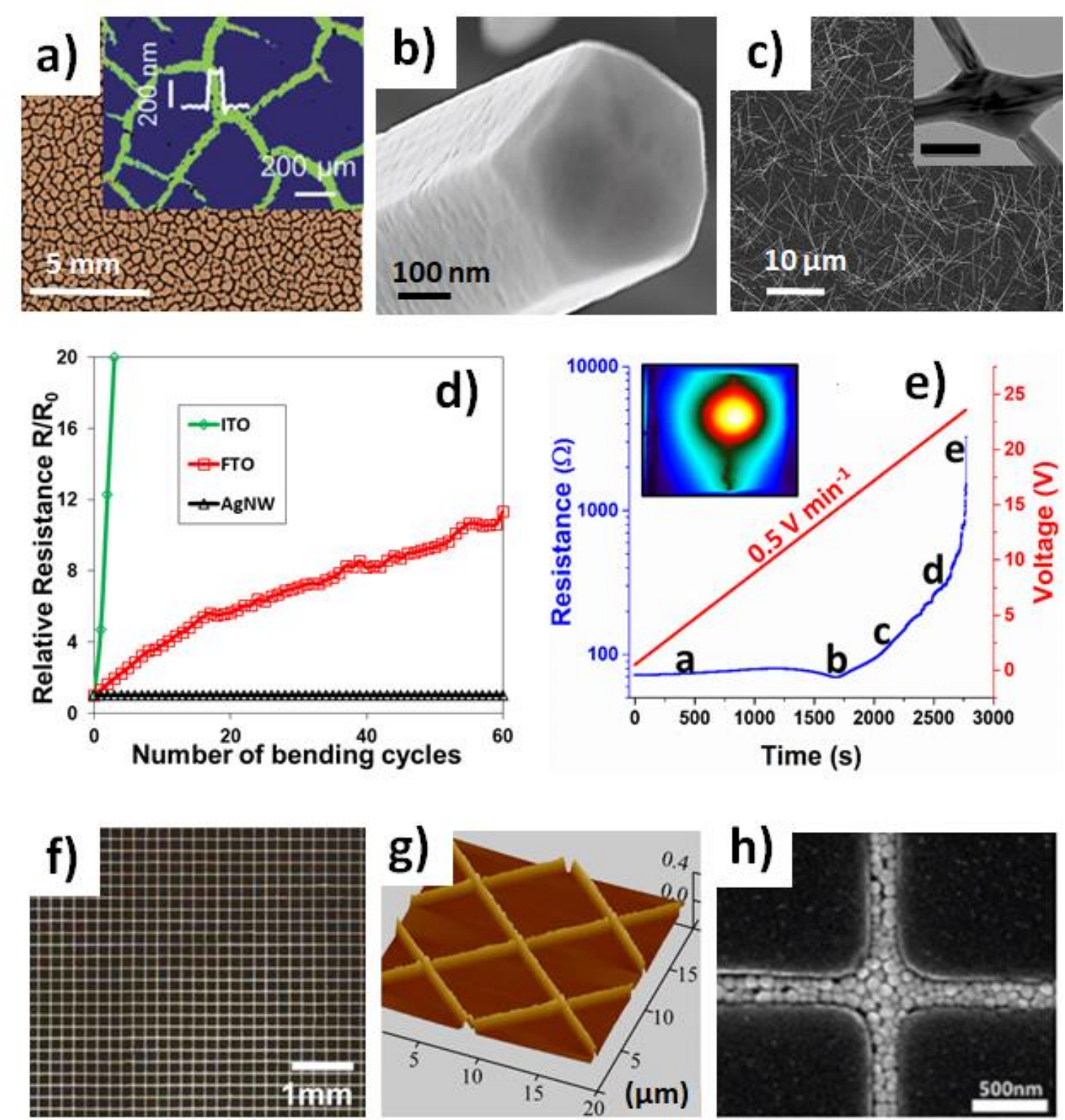

Figure 7. Main features of metallic-based THs. a) Optical image of an Ag mesh fabricated on glass; the inset corresponds to the optical profiler image (thickness of about $300 \mathrm{~nm}$ ). Reproduced with permission. ${ }^{[82]}$ Copyright 2014, Royal Society of Chemistry. b) High-resolution SEM image of a copper nanowire (CuNW) with a 5-fold symmetry. Reproduced with permission. ${ }^{[66]}$ Copyright 2017, John Wiley and Sons. c) Typical SEM picture of an AgNW random network. The inset shows a TEM picture of a sintered junction between two nanowires (bar scale: $100 \mathrm{~nm}$ ). ${ }^{[153]} \mathrm{d}$ ) Relative electrical resistance of different types of TH materials (ITO, FTO and AgNW) versus the number of bending cycles at $5 \mathrm{~mm}$ radius of curvature. Reproduced with permission. ${ }^{[20]}$ Copyright 2016, John Wiley and Sons. e) Evolution of the electrical resistance of an $\mathrm{AgNW}$ network during a voltage ramp of $0.5 \mathrm{~V} / \mathrm{min}$. The electrical resistance exhibits a drastic increase associated to the appearance and propagation of a crack, as revealed by the IR image in the inset (the width of the IR image corresponds to $25 \mathrm{~mm}$ ). Reproduced with permission. ${ }^{[93]}$ Copyright 2018, American Chemical Society. f) Optical microscopy image of a flexible transparent $\mathrm{Au}$ grid electrode with the following geometrical features: grid width: $4.5 \mu \mathrm{m}$; grid spacing: $200 \mu \mathrm{m}$; Au thickness: $\sim 90 \mathrm{~nm}$. Reproduced with permission. ${ }^{[154]}$ Copyright 2014, American Chemical Society. g) AFM profile sections of a silver grid fabricated by femtosecond laser writing of a silver aqueous solution. The profile shows a good uniformity of the grid. Reproduced with permission. ${ }^{[155]}$ Copyright 2016, AIP Publishing. h) SEM image of the silver nanoparticle (AgNP)-based metallic grid fabricated by capillary assembly of AgNPs. Reproduced with permission. ${ }^{[156]}$ Copyright 2016, Royal Society of Chemistry. 


\section{WILEY-VCH}

MNW networks have been the subject of a lot of research lately, with a focus on the industrial

786

787

788

789

790

791

792

793

794

795

796

797

798

799

800

801

802

803

804

805

806

807

808

integration of the networks as transparent electrodes into opto-electronic devices, as recently reviewed by several authors. ${ }^{[20,38,39,157,158]}$ Among the possible MNW materials, silver has been the most investigated ${ }^{[33,34,39,159-162]}$, while CuNW networks constitute an interesting alternative. ${ }^{[48,66,76,163,164]}$ Figure $7 \mathrm{~b}$ exhibits a high-resolution TEM image of a CuNW showing the 5-fold symmetry.${ }^{[66]}$ Figure $7 \mathrm{c}$ reports a typical SEM picture of a random AgNW network. The inset shows a TEM picture of a well-sintered junction between two AgNWs, ${ }^{[153]}$ knowing that an efficient local sintering of the junction between the different AgNWs leads to lower junction's electrical resistance and, therefore, to lower overall network electrical resistance. ${ }^{[90]}$ While the most used deposition techniques are spin-coating and spray-coating, several original methods have been proposed lately to fabricate AgNW networks with good performances. For instance, $\mathrm{Hu}$ et al. proposed a versatile agitation-assisted assembly approach that induces an orientation of AgNWs. This leads to excellent performances with a $R_{s h}$ value of $2.8 \Omega / \mathrm{sq}$, an optical transparency of $85 \%$ and a high stretchability of up to $40 \% .{ }^{[68]}$ The electrical resistance of junctions between AgNWs can be reduced by several methods, such as thermal annealing, ${ }^{[90]}$ UV treatment ${ }^{[165]}$ or as shown recently by Huang et al. using a self-limited nano-soldering method. This method can decrease $R_{s h}$ from 18.6 to $7.7 \Omega /$ sq while preserving a constant optical transmittance of $90 \% .{ }^{[166]}$ The first demonstration of AgNW networks being efficiently used as THs was published in 2012 by Celle et al.. ${ }^{[42]}$ These authors showed that the intrinsic features of AgNW random networks made it possible to combine bendability, transparency and high heating performances at low voltages (below $12 \mathrm{~V}$ ), which constitutes a clear asset for integration in many applications. ${ }^{[42]}$ This demonstration, on both glass and polymeric substrates, was followed by numerous publications. Sorel et al. ${ }^{[43]}$ published in 2014 a comprehensive physical approach of AgNW network-based THs, including the different 


\section{WILEY-VCH}

809 regimes associated with low network density (percolative regime) and high network density 810 (bulk-like regime). The authors also provided critical considerations related to electrical 811 conductivity, optical transparency and heat dissipation for efficient THs. Figure $7 \mathrm{~d}$ shows the 812 relative electrical resistance of different types of TH technologies (ITO, FTO and AgNW)

813 versus the number of bending cycles with a radius of curvature of $5 \mathrm{~mm}$. AgNW networks are 814 much more flexible than TCOs. ${ }^{[20]}$ However, one of the main issues with MNW networks is 815 their relative low stability under electrical and/or thermal stresses, even at device-operating conditions, as reported in several studies. ${ }^{[46,54,90,167,168]}$ This is illustrated in Figure 7e, which

817 displays the evolution of the electrical resistance of an AgNW network during a voltage ramp 818 of $0.5 \mathrm{~V} / \mathrm{min}$. The electrical resistance exhibits a drastic increase associated to the appearance 819 and propagation of a crack, as revealed by the IR image in the inset (the width of the IR image 820 corresponds to $25 \mathrm{~mm}$ ). ${ }^{[93]}$ As reported below (section 3.5.3), the deposition of a thin oxide 821 layer on top of MNW networks drastically enhances their stability or their adhesion. ${ }^{[47,48,167]}$ 822 These nanocomposites can lead to efficient and stable THs, ${ }^{[44,46,47,117,169]}$ as discussed further in section 3.5.

824 Metallic grids have been the subject of a lot of research since they appeared as promising 825 transparent electrodes, with both optical transmittance and sheet resistance being well controlled by adjusting the period and width/thickness of the patterns. ${ }^{[147,170]}$ The metal lines are thin enough $(\sim \mathrm{few} \mu \mathrm{m})$ to provide a low sheet resistance, while the period of the grid is 828 large enough $(\sim 5-200 \mu \mathrm{m})$ to provide sufficient transparency but not too large, to ensure heating uniformity over the entire TH. Figure $7 \mathrm{f}$ shows a typical optical image of a flexible transparent 830 Au grid electrode with the following geometrical features: grid width of $4.5 \mu \mathrm{m}$, grid spacing 831 of $200 \mu \mathrm{m}, \mathrm{Au}$ thickness of $\sim 90 \mathrm{~nm} \cdot{ }^{[154]}$ This Au grid electrode exhibits an optical transmittance 832 of $92 \%$ at $550 \mathrm{~nm}$ and a sheet resistance of $97 \Omega / \mathrm{sq}^{\left[{ }^{[154]}\right.}$ The fabrication of metal mesh electrodes was originally performed using vapor deposition processes, which require vacuum and generate

834 metal waste. Consequently, many studies have investigated other reliable fabrication methods, 


\section{WILEY-VCH}

835 and in particular large area printing methods like flexography, slot-casting, screen printing and others. Figure $7 \mathrm{~g}$ exhibits an AFM profile section of a silver grid, fabricated by femtosecond laser writing of a silver aqueous solution, which shows the good uniformity of the grid. ${ }^{[155]}$ This fabrication method possesses advantages in terms of flexibility and adaptability compared to alternative techniques like the combination of metal evaporation and lithography. Figure $7 \mathrm{~h}$

840 shows an SEM image of a silver nanoparticle-based (AgNP) metallic grid fabricated by 841 capillary assembly of AgNP. ${ }^{[156]}$ Of note, metallic grids can exhibit excellent flexibility. ${ }^{[147]}$

842 However, one of the potential weaknesses of metal grids is the weak adhesion to the substrate.

843 Lee et al proposed to enhance this adhesion through colloidal deposition and silver 844 enhancement steps. This improves the adhesion of the metal mesh thanks to an intermediate 845 adhesion layer based on 3-aminopropyltriethoxysilane. ${ }^{[12]}$ This approach results in an optical transmittance of $97.7 \%$ and a sheet resistance of $71.6 \Omega$ /sq, and its use as a TH enables to homogeneously reach a temperature of $245^{\circ} \mathrm{C}$ under an applied voltage of $7 \mathrm{~V}$, showing a clear enhancement of adhesion. ${ }^{[112]}$ Another very promising strategy to tackle adhesion issues relies

849 on embedded structures since they can: i/ enhance the surface smoothness, ii/ improve drastically the mechanical stability and iii/ induce strong adhesion to the substrate. Khan et al. demonstrated a cost-effective electrodeposition solution process of embedded metal-mesh with excellent mechanical, optical and heating performances. ${ }^{[74]}$ Of note, metallic fibers (with diameters much larger than nanowires) can exhibit interesting properties. Wang et al. recently

854 demonstrated that percolating networks of Ag nanofibers fabricated by blow-spinning 855 constitute efficient THs. ${ }^{[73]}$ This TH technology can indeed exhibit good optical and electrical properties, as demonstrated with Ag nanofibers networks that were obtained with an optical transmittance of $95 \%$ and a sheet resistance of $16 \Omega /$ sq, enabling to reach temperatures larger than $285^{\circ} \mathrm{C}$ with a $10 \mathrm{~V}$ bias. ${ }^{[73]}$ Singh et al. reported a $\mathrm{TH}$ with low operating voltage based on embedded PVA@Ag nanofiber network. This TCM structure has a smooth surface topology 


\section{WILEY-VCH}

861

862

863

864

865

866

867

868

869

870

871

872

873

874

875

876

877

878

879

880

881

882

883

884

885

case of copper based fibers, Jo et al. demonstrated a highly stretchable and transparent TH with ultra-low sheet resistance that can keep the working temperature constant up to $300 \%$ stretching. ${ }^{[76]}$

\subsection{Polymer-based materials}

Conductive polymers present attractive features for $\mathrm{TH}$ fabrication in terms of flexibility/stretchability, cost and processability. However, only few of them exhibit an electrical conductivity suitable for TH applications.

Recently, a breakthrough was performed in $\mathrm{TH}$ fabrication with the first demonstration of a $100 \%$ polymeric TH. Gueye et al. demonstrated the ability of poly(3,4-ethylenedioxythiophene) (PEDOT)-based thin films to be efficient THs, without the need for metal or conductive fillers. ${ }^{[50]}$ Three conductive polymers were studied (i) PEDOT doped with polystyrene sulfonate (PSS) and with ethylene glycol (EG): PEDOT:PSS-EG, (ii) PEDOT doped with trifluoromethanesulfonate $\mathrm{CF}_{3} \mathrm{SO}_{3}{ }^{-}$(OTf): PEDOT:OTf, and (iii) PEDOT:OTf thin films treated with dilute sulfuric acid to further enhance conductivity: PEDOT:Sulf. The chemical structures are displayed in Figure 8a. These conductive polymers show excellent optoelectronic and heating properties ${ }^{[50]}$, and are flexible, as shown in Figure $8 \mathrm{~b}$. The electrical resistance and heating properties of the polymer-based $\mathrm{TH}$ remain stable under strong mechanical stress (1000 cycles with a $9 \mathrm{~mm}$ bending radius), as shown in Figure 8c. Temperatures higher than $100{ }^{\circ} \mathrm{C}$ can be reached in a few minutes with a bias of less than $12 \mathrm{~V}$ (Figure 8d). Transparencies higher than $87 \%$ are generally obtained with extremely low light diffusion, the haze value being typically under $1 \%$. This aspect is very important because haziness is a critical parameter for most optoelectronic applications, and such low values are hardly ever achieved with other non-TCO materials. ${ }^{[38]}$ This polymer-based technology made it possible to reach high power densities, up to $10000 \mathrm{~W} / \mathrm{m}^{2}$ (Figure $8 \mathrm{~d}$ ). Heating homogeneity, 


\section{WILEY-VCH}

886

887

measured by IR imaging, and extended mechanical stability were demonstrated. Chemical instability could have been anticipated because PEDOT-based materials are known to be sensitive to light and moisture. However, after encapsulation with a barrier film, a loss of only $1^{\circ} \mathrm{C}$ was measured after $200 \mathrm{~h}$ under continuous heating at $55^{\circ} \mathrm{C}$, compared to $9^{\circ} \mathrm{C}$ without encapsulation, which indicates that encapsulation methods should be developed and optimized for the long term durability of these THs.

a)

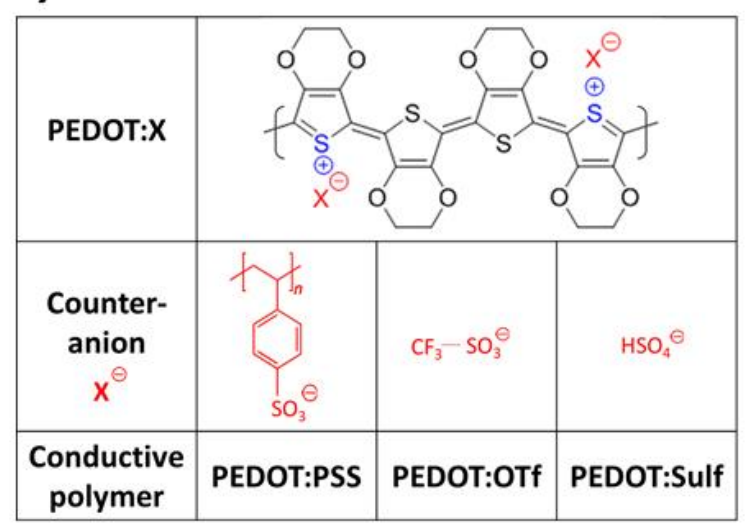

c)

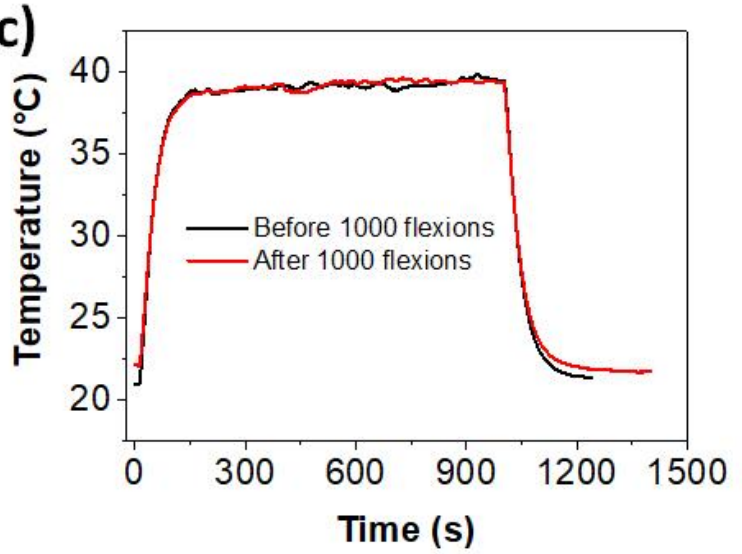

b)
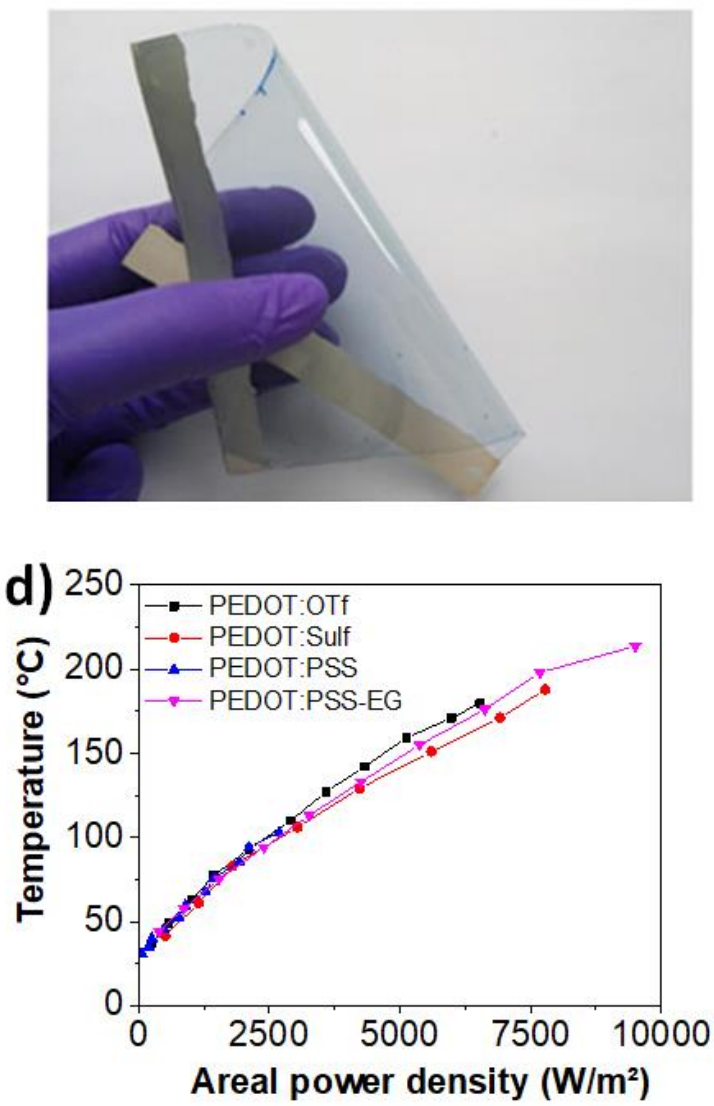

892

893

894

895

896

897

898

899

900

901

Figure 8. Main features of conductive polymer-based transparent heaters. a) Chemical structures of PEDOT and three main counter-ions forming respectively: PEDOT:PSS-EG, PEDOT:OTf and PEDOT:Sulf. b) Picture of a PEDOT-based TH illustrating its flexibility. c) Temperature increase of PEDOT:PSS-EG-based THs before and after 1000 bending cycles ( $9 \mathrm{~mm}$ radius of curvature) showing its good mechanical stability. d) Temperature increase versus areal power density for conductive polymer-based TH. b-d) Reproduced with permission. ${ }^{[50]}$ Copyright 2017, American Chemical Society.

Another recently reported approach is based on the use of nanofibers of conductive polymers to form a mesh, which can be used as a TH. An effective and cost-friendly method to produce such nanofibers is electrospinning, which allows the polymer to be deposited onto any curved or flexible surface. Some 


\section{WILEY-VCH}

903

904

905

906

907

908

909

910

911

912

913

914

915

916

917

918

919

920

921

922

923

924

925

926

927

928

experiments were carried out on polycaprolactone/carbon black (PCL/CB), polyethylene oxide/polyaniline (PEO/PANI) and PEO/PEDOT:PSS systems. ${ }^{[85,171]}$ The best results were obtained with the PEO/PEDOT:PSS blend. These polymeric THs show moderate performances in terms of heating properties, requiring a $60 \mathrm{~V}$ bias to reach $70{ }^{\circ} \mathrm{C}$, at $84 \%$ transparency. ${ }^{[85]}$

PEDOT:PSS films can also be patterned using a microfluidic post treatment with solvents. The conductivity is locally boosted by several orders of magnitude, making it possible to obtain localized heating with microscale resolution ${ }^{[172]}$.

These recent developments based on thin films of intrinsically highly conductive polymers open the way to new high performance and purely organic THs. ${ }^{[173]}$

\subsection{Nanocomposites and hybrid transparent heaters}

THs have gained a constantly increasing attention in research and industry these past years with a recent focus on multilayers, hybrids and nanocomposites. Matching materials with $\mathrm{TH}$ requirements is promising since it combines the best properties and advantages of each material, and generally offers much better performances than THs composed of a single material type. In other words, each conductive nanomaterial (TCO, metallic-based materials, carbon-based materials or conductive polymers) has its own advantages and disadvantages, but satisfying all industrial requirements with a single material is nearly impossible. The combination of several of these nanomaterials, however, can offer enhanced properties or better stability. This trend is observed in other modern domains in advanced materials science. This can be illustrated with the case of 1D carbon-based nanocomposites used in electrochemical energy storage devices; these have attracted intensive research interests lately, as an effective way to store energy from renewable energy sources. These aspects have been reviewed recently by Shi et al. ${ }^{[174]}$ who showed that 1D carbon-based nanocomposites (where CNT are either embedded, coated, encapsulated or supported) can be efficiently integrated into batteries and supercapacitors. 


\section{WILEY-VCH}

929 A large family of these hybrids for TH applications includes metallic nanowire networks or

930 grids/meshes coated with protective layers of graphene derivatives or TCOs. These composites

931 exhibit the very high electrical conductivity characteristic of metallic nanomaterials, and the

932 coatings offer higher electrical and thermal stability. Other structures include thin TCO layers

933 and metal nanowires ${ }^{[47]}$, or MNWs combined with conductive polymers ${ }^{[175]}$. The emerging

934 hybrids/nanocomposites based on nanomaterials open the way for flexible devices fabricated

935 using low-cost and up-scalable processes, and leads to a wide variety of modern applications

936 with futuristic shapes and everyday life uses. These nanomaterials can then be successfully

937 integrated into devices since they exhibit an interesting trade-off between electrical and

938 mechanical properties, stability, transparency and haze value. Figure 9 illustrates the richness

939 and diversity of the materials investigated for TH applications. These materials have been

940 classified according to the main investigated TH technologies: they are composed of either the

941 four primary material types (metallic oxides, metals, conductive polymers and carbon) or of

942 hybrids/nanocomposites composed the combination of these primary materials. The primary

943 material is the main material, while the secondary material is usually present in lesser amounts,

944 and generally added afterwards. The main materials used in each case (primary and

945 hybrids/nanocomposites) are represented in white letters. Each picture illustrates an example of

946 a hybrid category: (from left to right, clockwise) 1) metallic oxides/polymers, 2)

947 polymers/metals, 3) polymers/carbon, 4) carbon/metals, 5) metallic oxides/carbon, 6) metallic

948 oxides/metals. The color code used in Figure 9 applies to the rest of the figures of this review. 


\section{WILEY-VCH}

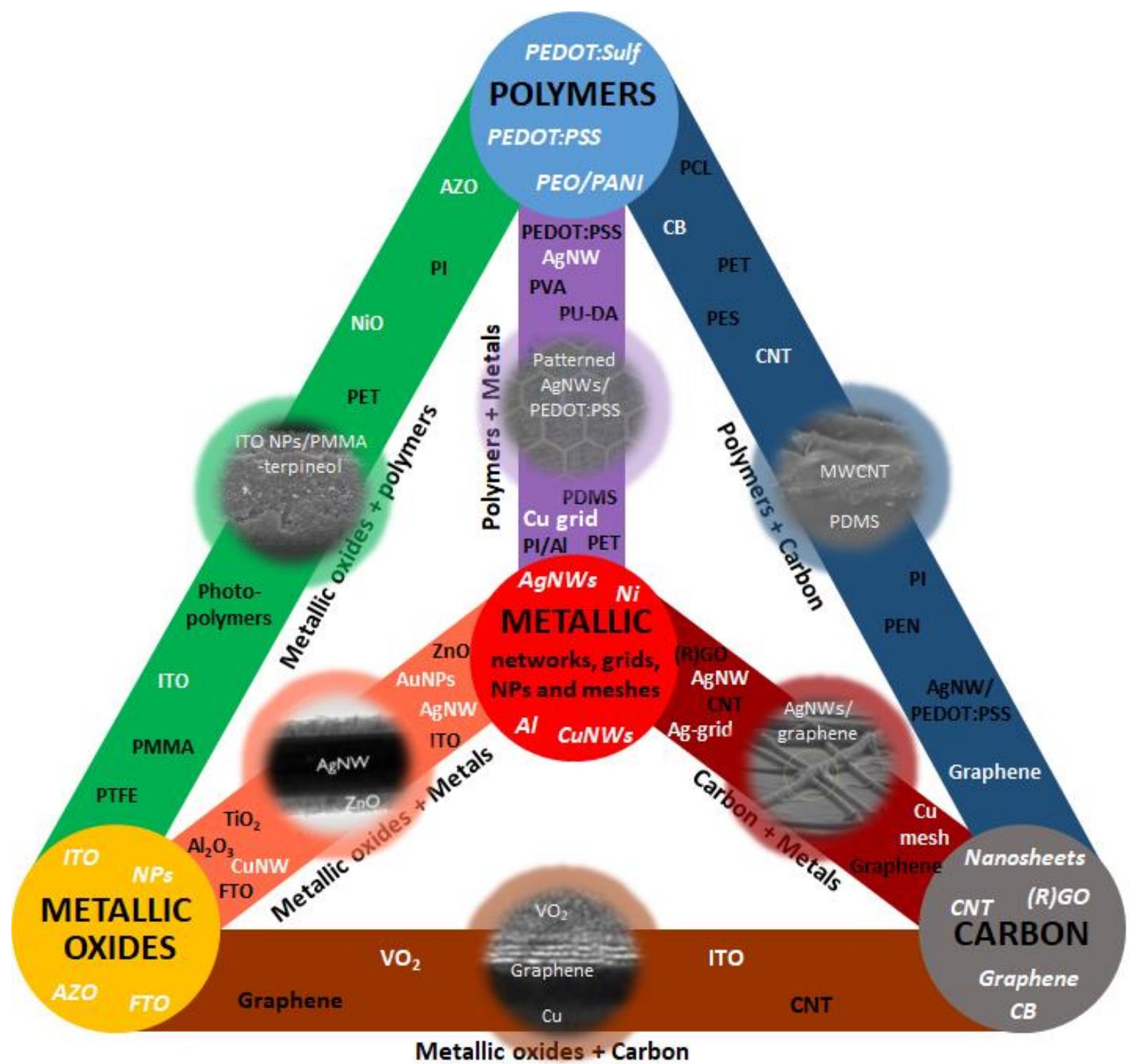

Metallic oxides + Carbon

950 Figure 9. Schematic classification of main TH technologies investigated: they are either composed of the four primary material types (metallic oxides, metals, conductive polymers and carbon) or of hybrids/nanocomposites through combinations. This illustrates the richness and diversity of TH-related advanced materials. The main materials used in each case (primary and hybrid) are represented in white letters. Each picture illustrates an example of a hybrid category: (from left to right, clockwise) 1) metallic oxides/polymers; SEM image of an ITO NP/polymethyl methacrylate (PMMA)-terpineol mixture. Reproduced with permission. ${ }^{[132]}$ Copyright 2015, Elsevier. 2) polymers/metals; optical micrograph of printed AgNW/PEDOT:PSS composite grids with an hexagonal pattern. Reproduced with permission. ${ }^{[176]}$ Copyright 2019, MDPI. 3) polymers/carbon; cross-section SEM images of a MWCNT/polydimethylsiloxane (PDMS) bilayer film. Reproduced with permission. ${ }^{[177]}$ Copyright

960 2015, Elsevier. 4) carbon/metals; Tilted cross-section SEM image of single-layer graphene/AgNW/glass. Reproduced with permission. ${ }^{[178]}$ Copyright 2019, John Wiley and Sons. 5) metallic oxides/carbon; HR-TEM of the cross section of graphene-supported $\mathrm{VO}_{2}$ on a copper substrate which will eventually be etched. Reproduced with permission. ${ }^{[53]}$ Copyright 2013, American Chemical Society. 6) metallic oxides/metals; TEM image of a $30 \mathrm{~nm}$ thick ZnO-coated AgNW. Reproduced with permission. ${ }^{[47]}$ Copyright 2018, American Chemical Society. 


\section{WILEY-VCH}

967 To show the recent evolution of $\mathrm{TH}$ technologies related either to emerging materials or to

968 nanocomposites, we have investigated the relative number of publications per TH technology. Figure

969 10a shows, through pie-charts, the percentage of scientific articles associated to the different $\mathrm{TH}$ 970 technologies, gathered every 4 years since 2004. This shows that before 2008 TCOs related to TH were

971 almost the only technology reported in the literature, while carbon materials (graphene and CNTs) were

972 starting to be well studied. From 2009 on, metallic nanowires, meshes and grids have attracted much

973 more attention. These past years, nanocomposites/hybrids were the focus of many articles, and excellent

974 performances and stability have been reported. Of note, conductive polymer-based THs started to be

975 explored around 2017. ${ }^{[50]}$ The growing size of the pie-charts with time illustrates the fact that the number

976 of articles per year has been multiplied by 10 between the first period (2004-2008) and the more recent

977 one (2014-2018). It clearly shows the growing interest in THs from the scientific community, driven by

978 the industrial needs. In parallel, there is a strong industrial activity in the TH field, which is not reflected

979 in scientific publications due to intellectual property strategies. Similar trends as those shown in Figure

980 10a can be surveyed in terms of patent application numbers linked to TH technologies.

981 To illustrate the versatility of nanocomposites, Figure 10b and 10c illustrate a typical example where

982 properties can be tuned if the AgNW content is varied in hybrid conductive films composed of CNTs

983 and AgNWs ${ }^{[102]}$ Figure 10b shows the influence of AgNW content on optical transmittance and haze

984 value, while Figure 10c shows the emissivity versus AgNW film content of similar film heaters prepared

985 by roll to-roll coating. ${ }^{[102]}$ Figure $10 \mathrm{~d}$ shows the enhancement of the stability of AgNW networks thanks

986 to thin oxide layer $(\mathrm{ZnO})$ coatings. The voltage failure observed during voltage ramps increases from 9

987 to $18 \mathrm{~V}$ for, respectively, bare AgNWs to $30 \mathrm{~nm} \mathrm{ZnO-coated} \mathrm{AgNWs,} \mathrm{showing} \mathrm{a} \mathrm{clear} \mathrm{electrical} \mathrm{stability}$

988 enhancement. ${ }^{[47]}$ 


\section{WILEY-VCH}

a) i) 2004-2008

ii) 2009-2013

iii) 2014-2018
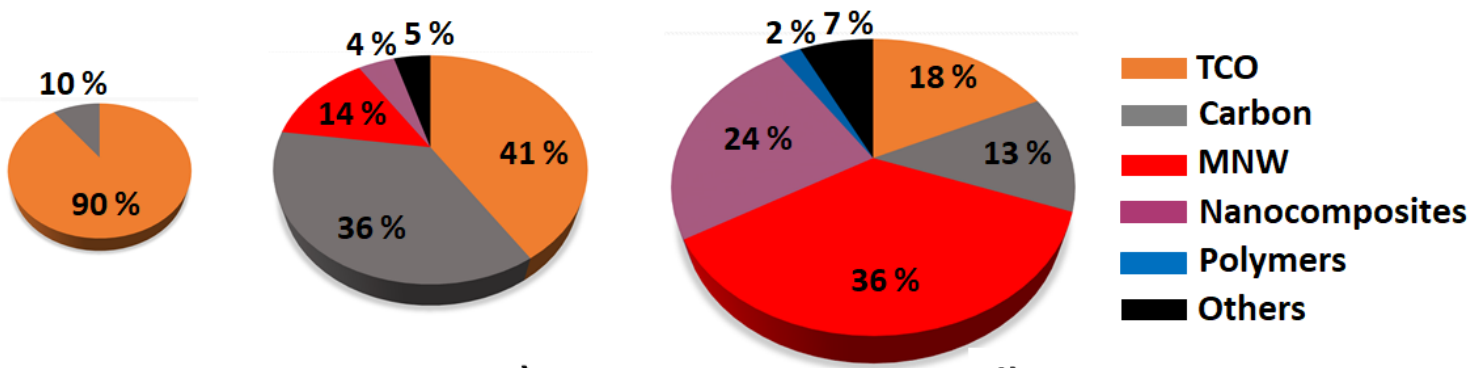

b)

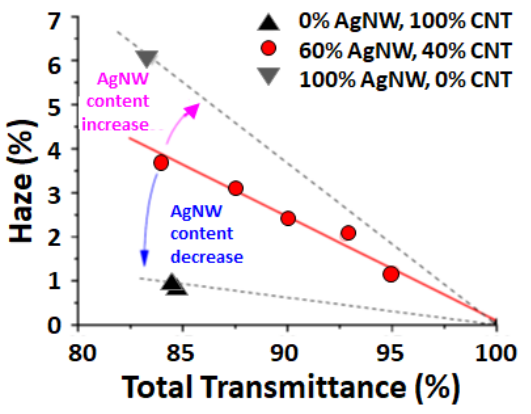

c)

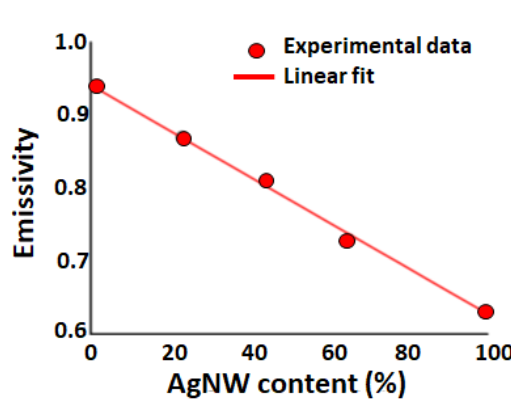

d)

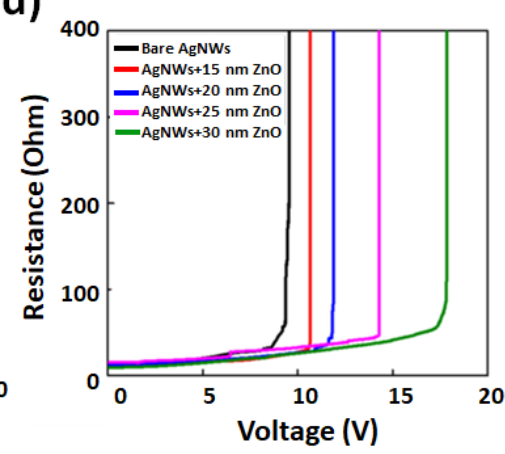

Figure 10. Emergence and examples of the assets of nanocomposite TH technologies. a) Pie-chart representing the percentage of scientific articles associated to the different $\mathrm{TH}$ technologies, showing that before 2008 mostly TCOs were mentioned in TH literature, while a small proportion of carbon materials (graphene and CNTs) were starting to be studied. Since 2009 MNW, meshes and grids have attracted much more attention. These past years, nanocomposites have been the focus of many articles, while conductive polymer-based THs have been explored since 2017. The pie-charts are growing with time to illustrate the fact that the number of articles per year has been multiplied by 10 between the first period (2004-2008) and the more recent one (2014-2018), demonstrating a clear growing interest in THs by the scientific community, driven by industrial needs (Sources: Scopus and Web of Science). b) Influence of AgNW content on optical transmittance and haze factor of hybrid conductive films composed of CNTs and AgNWs. c) Emissivity versus AgNW film content of similar film heaters prepared by roll to-roll coating. b,c) Reproduced with permission. ${ }^{[102]}$ Copyright 2013, Elsevier. d) Variation of electrical resistance versus voltage for bare and $\mathrm{ZnO}$-coated AgNW networks subjected to voltage ramps of $0.1 \mathrm{~V} / \mathrm{min}$ : the stability of the hybrid composite is clearly enhanced since the failure voltage increases with $\mathrm{ZnO}$ thickness. Reproduced with permission. ${ }^{[47]}$ Copyright 2018, American Chemical Society.

The next sections of 3.5 will focus in more detail on the properties of nanocomposites, discussed per combinations of materials.

\subsubsection{Higher thermal efficiency of metallic nanowire and carbon-based materials}

1011 Carbon-based materials and MNW hybrid materials have been widely developed for transparent

1012 properties enhancements like haze tuning ${ }^{[102,179,180]}$, but also to improve stability ${ }^{[181-183]}$ or

1013 adhesion. ${ }^{[184]}$ These hybrids are mainly comprised of 2D MNW networks (copper or silver) 


\section{WILEY-VCH}

1014 combined with CNTs, graphene, GO or rGO. As reported in section 3.2, carbon-based THs

1015 suffer from their poor conductivity at high levels of transparency. For heating applications,

1016 strong synergies are evidenced when combining the properties of carbon materials and MNWs

1017 for efficient, stable and highly flexible THs.

1018 The carbonaceous species (CNTs or Graphene) of the NW-based hybrids significantly improves

1019 stability and heat dissipation ${ }^{[116]}$ of the MNW networks for better thermal efficiency. ${ }^{[185]}$ Indeed,

1020 a more effective spreading of the heat with a small amount of SWCNTs improves the overall

1021 thermal stability of the NW network by improving resistance to current shocks. ${ }^{[185]}$ Moreover,

1022 the excellent thermal contact between nanowires and graphene, estimated at $0.5 \mathrm{~m} . \mathrm{K} / \mathrm{W}$ by

1023 Gupta el al. prevents Joule heating at the NW/NW junctions. ${ }^{[186]}$ Sadeque et al. focused their

1024 work on thermal transport behaviors in 2D networks of hybrid materials, especially 1025 graphene/AgNW composites. ${ }^{[187]}$ They disentangled contributions from local self-heating

1026 (hotspots) and heat spreading from the contacts, using thermoreflectance measurements. ${ }^{[187]}$

1027 Figure 11a shows SEM pictures of a CNT-AgNW hybrid heater. Highly stretchable and stable

1028 hybrid THs were developed, with a very low haze (under 1\% at 95\% transparency) and elasticity

1029 as high as $20 \%{ }^{[80]}$ As reported very recently by Wang et al., a tri-layer film-based heater of

1030 rGO-AgNW-GO, as shown in Figure 11b, exhibits far better chemical, mechanical and thermal

1031 performances than pure AgNWs. ${ }^{[188]}$ They report a significant improvement in long-term

1032 stability, with fewer failures on the sandwich-structured film heater. 


\section{WILEY-VCH}

a) i)

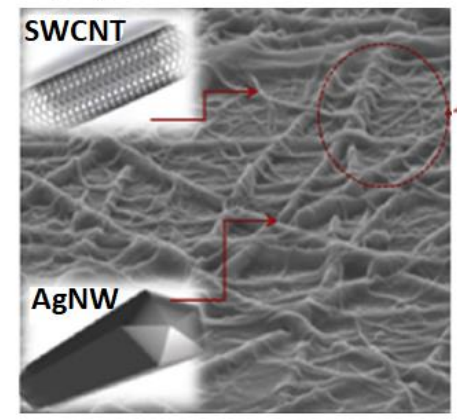

b)

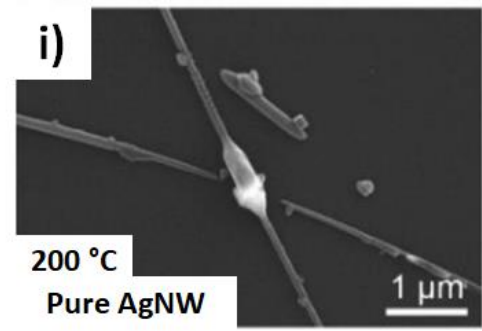

ii)

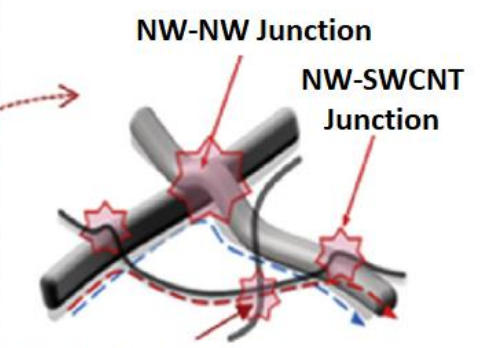

SWCNT-SWCNT Junction iii)
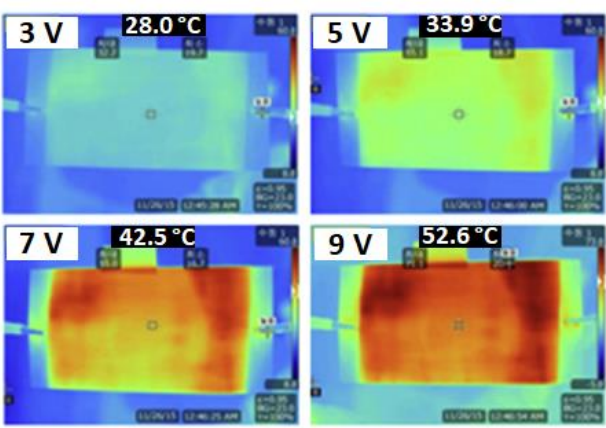

iii)

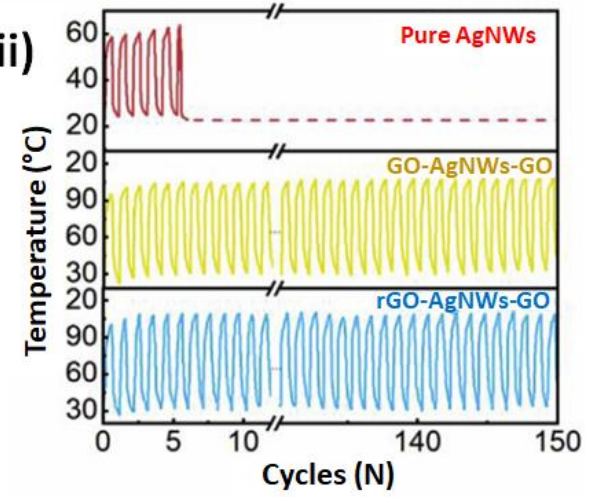

Cycles (N)

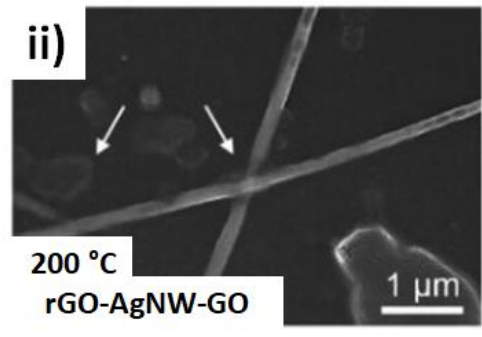

rGO-AgNW-GO
1034

1035

1036

1037

1038

1039

1040

1041

1042

1043

1044

1045

1046

1047

1048

1049

1050

1051

1052

Figure 11. Carbon and MNW-based hybrid THs. a) AgNW-SWCNT hybrid composite layer on TPU: i) FESEM surface image of the hybrid composite layer. ii) schematic representation showing the current pathways. iii) IR thermal images of the SWCNT-AgNW hybrid TH, with a sheet resistance of $30 \Omega /$ sq, under different applied input voltages (3, 5, 7 and 9 V). Reproduced with permission. ${ }^{[80]}$ Copyright 2018, Elsevier. b) Tri-layer rGO-AgNW-GO composite film i) SEM pictures of pure AgNWs, ii) tri-layer composite film heated at $200^{\circ} \mathrm{C}$, iii) heating and cooling tests of pure AgNW, GO-AgNW-GO and rGOAgNW-GO THs under ON/OFF current cycles of $60 \mathrm{~s}$. Reproduced with permission. ${ }^{[188]}$ Copyright 2019, Elsevier.

\subsubsection{Adhesion enhancement within metallic nanowire/polymer hybrid transparent}

\section{heaters}

As previously mentioned, polymers are extremely attractive in terms of flexibility/stretchability and processability. Nanocomposite materials relying on MNWs and polymers combine the high conductivity of a MNW network with the desirable mechanical properties of polymers, like flexibility, ${ }^{[189]}$ stretchability, ${ }^{[190]}$ and adhesion ${ }^{[175]}$.

Thanks to the addition of conducting elements in polymer matrices, it is possible to enhance the conductivity while preserving processability. Most of these composite-based THs take advantage of metallic nanofillers, often made of silver or copper. Nanospecies with high form factors are preferred, to favor percolation for the electron pathways. In particular, metallic 


\section{WILEY-VCH}

1053 nanowires can provide efficient networks at low densities, as described by the stick percolation

1054 theory. ${ }^{[191,192]}$ PEDOT:PSS, Polyimide (PI), polydimethylsiloxane (PDMS), PET, polyester

1055 (PE), poly(methyl methacrylate) PMMA, and even healable polyurethane (PU-DA) are the

1056 most reported matrices for the fabrication of composites for THs. ${ }^{[79,112,114,175,189,193-204]}$

1057 Biopolymers like chitosan are interesting emerging materials for medical $\mathrm{TH}$ applications

1058 because of their biocompatibility and biodegradability. ${ }^{[205]}$ For composites, the choice of the

1059 polymer-conducting filler couple depends on the specific properties that are targeted. Polymers

1060 generally act as binders to ensure good contacts between nanoparticles and substrate, or they

1061 can serve as flexible/stretchable substrates to provide these properties to the system. They do

1062 not improve the heating properties, but are interesting nonetheless because of their optical

1063 properties (thin film transparency), their potential ability to allow the homogeneous dispersion

1064 of conducting nanoparticles, and for their mechanical performances. PEDOT:PSS is also often

1065 used as a host for the fabrication of conducting composites, and has mainly been used to

1066 improve the adhesion with the substrate. The comparison of film adhesion before and after

1067 peeling of 3M Scotch tape is demonstrated in Figure 12a and 12b for a pristine AgNW (Figure

1068 12a) and PEDOT:PSS composite film (Figure 12b). ${ }^{[175]}$ The optical transmittance (measured at

$1069550 \mathrm{~nm}$ ) was increased from $75 \%$ to $81 \%$ for the $\mathrm{AgNW}$ film, while it remains at $79 \%$ for the

1070 composite film, demonstrating the enhanced adhesion of the composite.

1071 PEDOT:PSS can also enhance the conductivity by creating conductive pathways between

1072 spatially separated nanowires. ${ }^{[206]}$ In some cases, the conductive polymer can be doped with

1073 graphene to improve heating stability. ${ }^{[116]}$

1074 Nearly all reported polymer/AgNW hybrid systems show similar performances with a maximal 1075 temperature of $100-150{ }^{\circ} \mathrm{C}$, a transparency around $85 \%$ at an operating voltage between 10 and

$107640 \mathrm{~V}$. Large differences in haze factor are observed, with values ranging from $3 \%{ }^{[189]}$ to 30

$1077 \% .{ }^{[194]}$ Screen printing, doctor-blading and drop coating are the main methods reported for the

1078 deposition of AgNWs, which are then coated with the polymer. ${ }^{[175,189,194]}$ AgNWs can be 


\section{WILEY-VCH}

1079 embedded to improve the contact and stability in flexion, with a stable resistance after 1000

1080 bending cycles (with a $500 \mu \mathrm{m}$ radius of curvature). ${ }^{[189]}$ Another way to stabilize the system is

1081 to add a thin transparent protective layer of poly(vinyl alcohol) (PVA) or insoluble PI. ${ }^{[194,196]}$

1082 Healable polymers, based on the Diels-Alder reaction, can be cured by simple heat

1083 treatment. ${ }^{[202]}$ Figure $12 \mathrm{c}$ shows that after deliberate damage by a knife cut, the

$1084 \mathrm{AgNW} /$ polyurethane-based TH can heal and recover its original conductivity thanks to a simple

1085 heat treatment at $120^{\circ} \mathrm{C} .{ }^{[202]}$

1086 Hybrid materials with a copper/alumina/PI combination are interesting, with a high and stable

1087 temperature of $300^{\circ} \mathrm{C}$ reached for a $10 \mathrm{~V}$ bias, associated with $91 \%$ transparency. ${ }^{[201]}$ Alumina

1088 limits the diffusion and oxidation of $\mathrm{Cu}$, while $\mathrm{PI}$ is an effective binder to improve the adhesion

1089 strength between the $\mathrm{Cu}$ wires and the substrate. Heating stability was demonstrated for 100

1090 cycles with a substantial pulsed current of 1.5 A. Mechanical properties were investigated with

1091 bending (1000 cycles, 2 mm radius) and stretching (30\% stretchability on PDMS) experiments,

1092 and no significant change in conductance was observed. Similar improvement of adhesion

1093 properties of AgNW networks to the substrate due to the use of PI was demonstrated by Lu et 1094 al. ${ }^{[204]}$ 


\section{WILEY-VCH}

a)

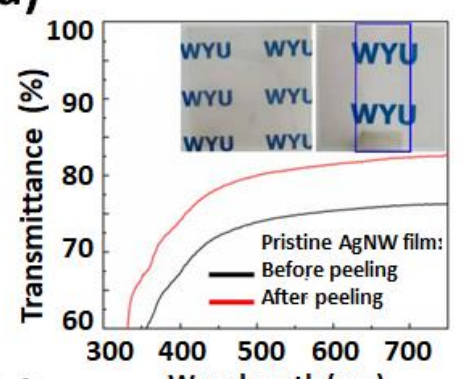

b)

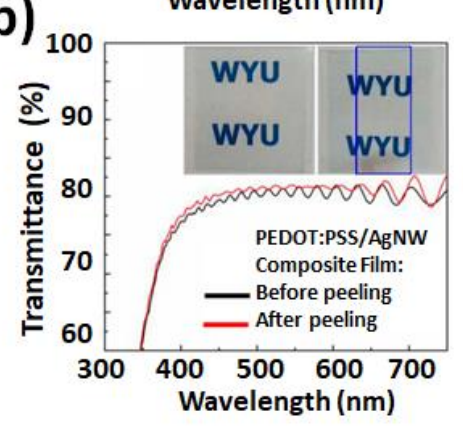

c)
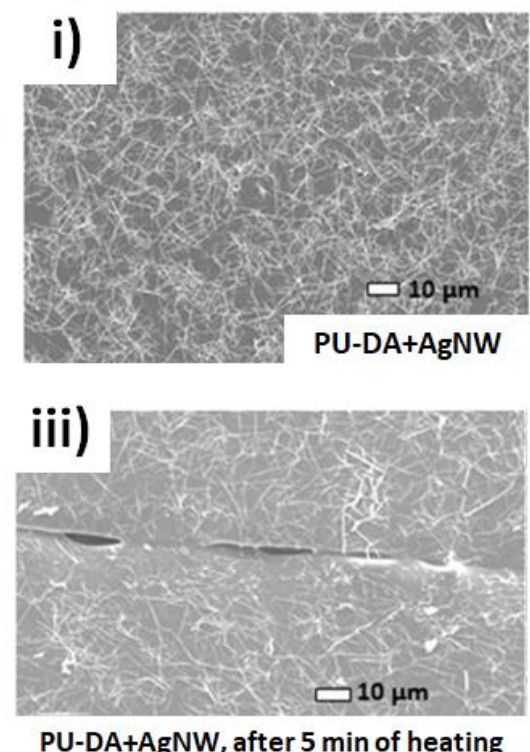

PU-DA+AgNW, after 5 min of heating
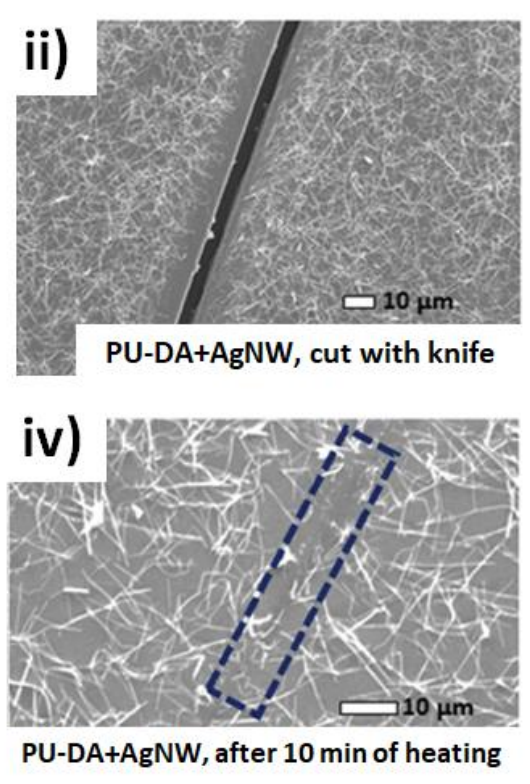

1095

1096

1097

1098

1099

1100

1101

1102

1103

1104

1105

1106

1107

1108

1109

1110

1111

1112

1113

1114 the networks $\left.{ }^{[90]}\right)$. Furthermore, electrical instability is observed when the networks undergo 1115 electrical stress ${ }^{[93,168]}$. CuNW networks are interesting ${ }^{[30,31]}$ since the price of $\mathrm{Cu}$ is much lower

1116 than $\mathrm{Ag}$. However, $\mathrm{Cu}$ is much more prone to oxidation than $\mathrm{Ag}$ and thus the chemical stability

Figure 12. THs based on nanocomposites with AgNW networks and polymers. a) Peeling test of a pristine AgNW film showing a large increase in optical transparency. b) Similar experiment with AgNW and PEDOT:PSS composite showing no increase in optical transparency, associated to a clear improvement in adhesion thanks to the polymer. Reproduced with permission. ${ }^{[175]}$ Copyright 2017, MDPI. c) FESEM images of the transparent, flexible and healable material with AgNWs and polymers: i) The healable $\mathrm{TH}$ as deposited, ii) after a cut with a knife, iii) healing of the electrode at a temperature of $120^{\circ} \mathrm{C}$ for $5 \mathrm{~min}$, and (iv) healing of the electrode at a temperature of $120^{\circ} \mathrm{C}$ for $10 \mathrm{~min}$. The dotted box indicates the location of the previous cut. Reproduced with permission. ${ }^{[202]}$ Copyright 2017, Royal Society of Chemistry.

\subsubsection{Stability enhancement of metallic nanowires with oxide material}

Percolating MNW networks have been shown to act as efficient transparent electrodes ${ }^{[20,33,34]}$ and can be used in solar cells ${ }^{[40]}$ and as $\mathrm{THs}^{[42]}$. The most studied MNWs are AgNWs since their synthesis and properties exhibit strong advantages: silver is the material with the highest thermal and electrical conductivity at room temperature, and the synthesis of AgNWs is wellmastered. However, these metallic nanowire networks suffer from stability issues. MNW thermal instability leads to spheroidization (associated to the loss of the percolating nature of

than Ag. However, Cu is much more prone to oxidation than Ag and thus the chemical stability 


\section{WILEY-VCH}

1117 of CuNWs is a more critical issue. To enhance the stability of MNWs in general, several

1118 solutions have been investigated. The coating of MNWs by a thin oxide layer has shown clear 1119 improvements in stability. This strategy has been reported for $\mathrm{AgNW} / \mathrm{TiO}_{2}{ }^{[46]}$, $1120 \mathrm{AgNW} / \mathrm{ZnO}^{[47,207]}, \mathrm{CuNW} / \mathrm{Al}_{2} \mathrm{O}_{3}{ }^{[48,169]}, \mathrm{CuNW} / \mathrm{ZnO} \cdot{ }^{[208]}$ Figure 13 shows SEM (a,b) and TEM $1121(\mathrm{c}, \mathrm{d})$ images of AgNW networks before $(\mathrm{a}, \mathrm{c})$ and after $(\mathrm{b}, \mathrm{d})$ the deposition of a thin layer of $1122 \mathrm{ZnO}$ using atmospheric pressure spatial atomic layer deposition (AP-SALD) ${ }^{[47]}$. This technique 1123 appears as an ideal approach to protect MNWs since it is a rapid, atmospheric and cheap method 1124 for thin film conformal deposition, and therefore does not compromise the low-temperature, 1125 atmospheric pressure fabrication of MNW networks. ${ }^{[208-210]}$ A $25 \mathrm{~nm}$ thick coating of $\mathrm{ZnO}$ 1126 drastically enhances the stability of the TH, as shown in Figure $13 \mathrm{e}^{[47]}$ Figure $13 \mathrm{f}$ shows the 1127 relative electrical resistance of the bare $\mathrm{AgNW}$ network and the $\mathrm{ZnO}$ : $\mathrm{Al} / \mathrm{AgNW}$ nanocomposite 1128 versus time when the samples were subjected to voltage ramp cycles (between $2 \mathrm{~V}$ and $6.5 \mathrm{~V}$ ).

1129 The electrical stability is clearly enhanced thanks to the conformal coating when compared to 1130 bare AgNWs. ${ }^{[208]}$ Similarly, Tigan et al. recently reported an extensive study relating CuNW 1131 network density and oxide nature $\left(\mathrm{Al}_{2} \mathrm{O}_{3}, \mathrm{ZnO}\right)$ with electrical and optical properties, as well 1132 as stability. These authors were able to reach thermal stability up to $273{ }^{\circ} \mathrm{C}$ for CuNW with a 1133 thin $\mathrm{Al}_{2} \mathrm{O}_{3}$ coating, and a remarkably high heating rate of $14{ }^{\circ} \mathrm{C} / \mathrm{s} .{ }^{[169]}$

1134 In addition to improving the network stability, oxide coatings give rise to materials with a much

1135 higher adhesion than bare nanowires. Figure 13g shows an optical picture of the peeling off test 1136 of a CuNW/Al ${ }_{2} \mathrm{O}_{3} /$ polyimide(PI) composite film using $3 \mathrm{M}$ scotch tape. ${ }^{[201]}$ Figure $13 \mathrm{~h}$ reports 1137 the variations in optical transmittance (blue) and sheet resistance (black) for $\mathrm{Cu}$ wire $/ \mathrm{Al}_{2} \mathrm{O}_{3} / \mathrm{PI}$ 1138 (black circles) and $\mathrm{Cu}$ wire/ $\mathrm{Al}_{2} \mathrm{O}_{3}$ (black square) network composite films versus the number 1139 of performed tape tests. The composite film $\left(\mathrm{Cu}\right.$ wire $\left./ \mathrm{Al}_{2} \mathrm{O}_{3} / \mathrm{PI}\right)$ clearly exhibits an enhanced 1140 adhesion compared to the $\mathrm{Cu}$ wire $/ \mathrm{Al}_{2} \mathrm{O}_{3}$, which became insulating after the third tape test. ${ }^{\text {[201] }}$

1141 Of note, an approach has recently been used to enhance the transmittance of AgNW networks 1142 coated with protective metal oxide bilayers thanks to an anti-reflective coating. ${ }^{[211]}$ 


\section{WILEY-VCH}

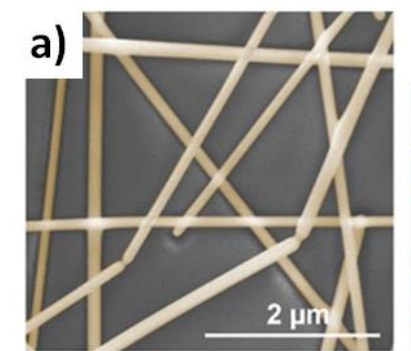

e)

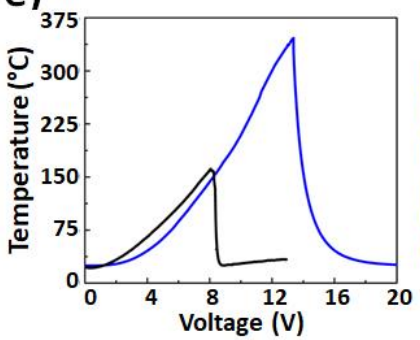

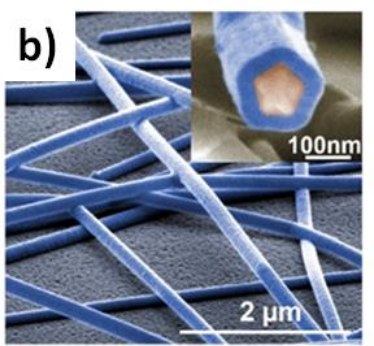

f)

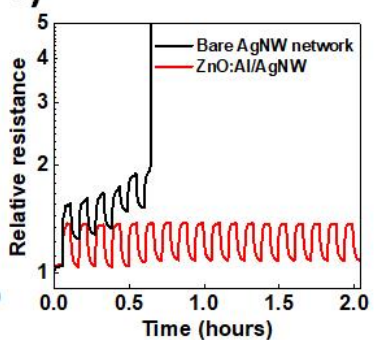

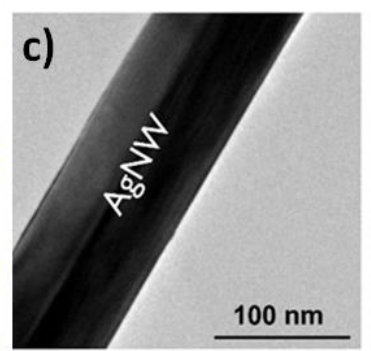

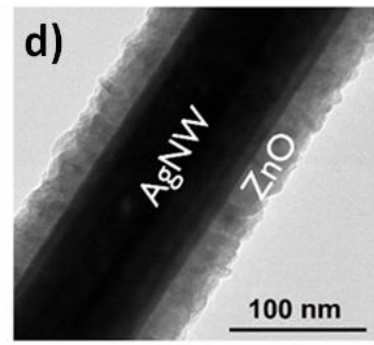

g)

h)
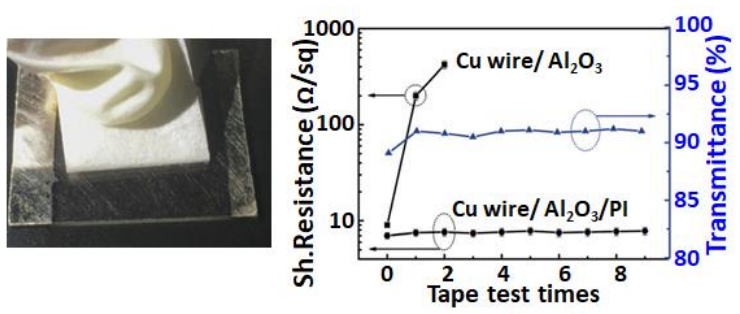

1143

1144

1145

1146

1147

1148

1149

1150

1151

1152

1153

1154

1155

1156

1157

1158

1159

1160

1161

1162

1163

1164

1165

1166

1167

Figure 13. THs based on nanocomposites of MNWs and thin oxide layers. SEM (a,b) and TEM (c,d) observations of $\mathrm{AgNW}$ before $(\mathrm{a}, \mathrm{c})$ and after $(\mathrm{b}, \mathrm{d})$ deposition of a thin layer of $\mathrm{ZnO}$ layer using atmospheric pressure spatial atomic layer deposition (AP-SALD). A $25 \mathrm{~nm}$ thick $\mathrm{ZnO}$ coating drastically enhances the electrical stability of the $\mathrm{TH}$, as shown in e), with the measured temperature during a voltage ramp for a bare and a coated silver nanowire network, in black and blue lines respectively. a-e) Reproduced with permission. ${ }^{[47]}$ Copyright 2018, American Chemical Society. f) Relative electrical resistance versus time of the bare $\mathrm{AgNW}$ network and $\mathrm{ZnO}$ : $\mathrm{Al} / \mathrm{AgNW}$ nanocomposite when the samples were subjected to voltage ramp cycles (between $2 \mathrm{~V}$ and $6.5 \mathrm{~V}$ ): the electrical stability is enhanced thanks to the conformal coating. Reproduced with permission. ${ }^{[208]}$ Copyright 2019 , Royal Society of Chemistry. g) Optical picture showing the peeling off test for the $\mathrm{Cu}$ wire/ $\mathrm{Al}_{2} \mathrm{O}_{3} /$ polyimide(PI) composite film by $3 \mathrm{M}$ scotch tape.h) Variations of sheet resistance (black) versus the number of performed tape tests for $\mathrm{Cu}$ wire/ $/ \mathrm{Al}_{2} \mathrm{O}_{3} / \mathrm{PI}$ and for $\mathrm{Cu}$ wire $/ \mathrm{Al}_{2} \mathrm{O}_{3}$ network composite films; optical transmittance values (blue) $\mathrm{Cu}$ wire $/ \mathrm{Al}_{2} \mathrm{O}_{3} / \mathrm{PI}$ are also plotted. g,h) Reproduced with permission. ${ }^{[201]}$ Copyright 2016, Royal Society of Chemistry.

While the positive effects of thin oxide coatings on the performance of MNW networks have been reported several times, coating with metallic or oxide nanoparticles (NP) can also be an efficient method. For example, Morgenstern et al. showed that full encapsulation of the AgNW network by a layer of ZnO-NP drastically enhances thermal stability. ${ }^{[207]}$ Cheong demonstrated that $\mathrm{AgNW}$ and $\mathrm{AZO}$ can lead to efficient and flexible THs: the AZO coating enables to reach a higher average film temperature of $100{ }^{\circ} \mathrm{C}\left(30^{\circ} \mathrm{C}\right.$ higher than uncoated $\left.\mathrm{AgNWs}\right)$ as well as a greater heating uniformity. ${ }^{[212]}$ Cheng et al. also showed that AgNWs can be decorated with gold nanoparticles, leading to a greatly enhanced thermal stability. ${ }^{[213]}$ Sharma et al. demonstrated that highly stable THs can be obtained with AgNWs decorated with cobalt 


\section{WILEY-VCH}

1168 nanoparticles (CoNPs). It was found that an optimum AgNW-CoNP ratio of 50-50 wt\% leads

to good performances of the corresponding $\mathrm{TH}$, with a thermal stability up to $350{ }^{\circ} \mathrm{C} .{ }^{[214]}$

\subsubsection{Metallic grids and transparent conductive oxides or graphene}

1172 Hybrids based on metallic grids combined with graphene exhibit a superior electrical and

1173 mechanical stability. Kim et al. ${ }^{[148]}$ fabricated copper grid meshes by photolithography and

1174 deposited a graphene layer on top of the meshes (Figure 14a), while Kang et al. ${ }^{[215]}$ used

1175 electro-hydrodynamic jet printing to write silver grids on a large-area graphene layer. In both

1176 cases the hybrids showed improved and more uniform heating performances ${ }^{[215]}$ compared to

1177 each separate material, even after thousands of bending or twisting cycles. ${ }^{[148]}$ In the case of the

$1178 \mathrm{Cu}$ grid/graphene, the excellent flexibility is also due to the corona treatment of the PET

1179 substrate. Another study on metallic grid-based hybrid THs combined Ag meshes with a thin

1180 layer of ITO (Figure 14b). In this study, Kwon et al showed a $12 \%$ increase in optical 1181 transmittance, followed by a lower power consumption compared to the as-deposited ITO 1182 films ${ }^{[149]}$.
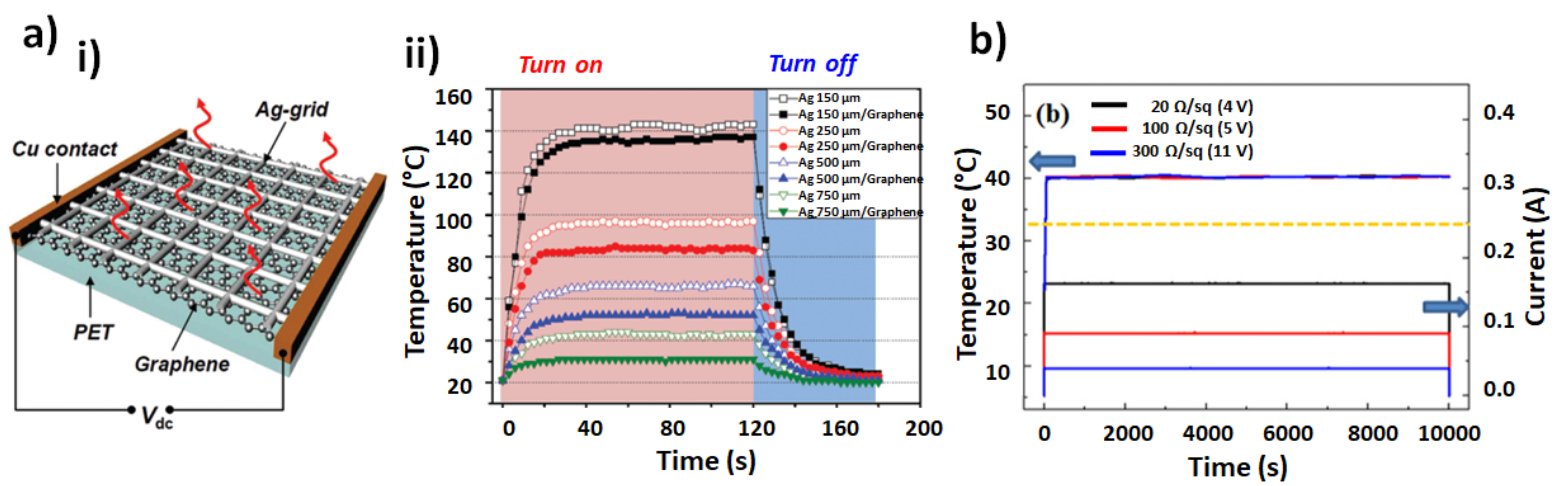

Figure 14. Transparent heaters from metallic grid-based hybrids. a) Ag-grid/graphene heaters: i) schematic illustration of the Ag-grid/graphene film heater connected to $\mathrm{Cu}$ electrodes. The size of the heating area was $5 \times 5 \mathrm{~cm}^{2}$ and ii) time-dependent temperature response of the $\mathrm{Ag}$-grid and $\mathrm{Ag}$-grid coated with graphene heaters. Reproduced with permission. ${ }^{[215]}$ Copyright 2015, Royal Society of Chemistry. b) Ag mesh-ITO hybrid heaters with sheet resistances of 20, 100, and $300 \Omega / \mathrm{sq}$. The temperature remains very stable for 10,000 s while a voltage of 4,5 and 11 volts is applied, respectively. Reproduced with permission. ${ }^{[149]}$ Copyright 2014, IOP Publishing. 


\section{WILEY-VCH}

$1192 \quad$ 3.5.5 Transparent heaters based on metallic or transparent conductive oxide multilayers

1193 Other combinations of materials have been reported in the literature, including multilayers of

1194 TCOs or polymers with metallic thin films, or by combining different TCOs and nanoparticles.

1195 Several studies deal with the combination of a metallic thin film that is sandwiched between

1196 two TCO layers. Kim's group demonstrated that it is possible to make high-performance and

1197 flexible THs using continuous roll-to-roll (R2R) sputtering. ${ }^{[51]}$ In their studies, $\mathrm{Ag}$ or $\mathrm{Cu}$ was

1198 sputtered, from metallic targets, in between $40 \mathrm{~nm}$ thick ITO layers. They used a pilot scale

1199 system capable of coating $700 \mathrm{~mm}$ wide PET substrates at room temperature. In the case of

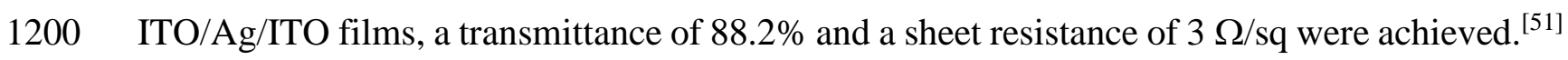

$120150 \times 50 \mathrm{~mm}^{2} \mathrm{THs}$ were then fabricated, reaching a steady-state temperature of $100{ }^{\circ} \mathrm{C}$ by

1202

applying $5 \mathrm{~V}$. In this case, the Ag layer was $12 \mathrm{~nm}$ thick and took around $100 \mathrm{~s}$ to reach 100

1203

${ }^{\circ} \mathrm{C}$. In the case of ITO/Cu/ITO layers, an optical transmittance of $73.9 \%$ and a sheet resistance

1204

of $11.8 \Omega /$ sq were obtained. ${ }^{[52]}$ In this study, the best figure of merit was obtained for $12 \mathrm{~nm}$

1205 thick $\mathrm{Cu}$ layers, and temperatures above $100{ }^{\circ} \mathrm{C}$ were obtained under a bias of $8 \mathrm{~V}$. In another study, the ITO layers were replaced by InZnSiO, in combination with Ag, using the same R2R sputtering approach. ${ }^{[216]}$ The electrodes showed a transmittance of $91.91 \%$ and a sheet resistance of $7.83 \Omega / \mathrm{sq}$ for a $8 \mathrm{~nm}$ thick Ag layer. The electrodes reached $110{ }^{\circ} \mathrm{C}$ for a bias of

1209 only $2.4 \mathrm{~V}$. The amorphous nature of the oxide yielded a high thermal stability upon cycling, as well as mechanical stability (no degradation of performance after 10,000 bending tests with

1211 a $10 \mathrm{~mm}$ bending radius). ${ }^{[216]}$ The same group presented a similar approach in which ITO was

1212 replaced by $\mathrm{SnO}_{2}$ and the metal layer was composed of a $\mathrm{AgPdCu}$ alloy. ${ }^{[217]}$ In this case, these

1213 flexible In-free electrodes were deposited by thermal evaporation, and showed a minimum sheet

1214 resistance of $9.42 \Omega /$ sq with transmittance values above $91 \%$ for $50 \mathrm{~nm}$ thick $\mathrm{SnO}_{2}$ layers and

1215 a $10 \mathrm{~nm}$ thick metallic layer. ${ }^{[217]}$ The metallic layers alone showed a poorer performance than 1216 the tri-layer in terms of both conductivity and transmittance, due to the antireflective effects of 


\section{WILEY-VCH}

1217 the multiple coatings. THs made of $\mathrm{SnO}_{2} / \mathrm{AgPdCu} / \mathrm{SnO}_{2}$ multilayers reached different

1218 temperatures for different $\mathrm{SnO}_{2}$ thicknesses. For $10 \mathrm{~nm}$ thick oxide layers, temperatures above

$1219110{ }^{\circ} \mathrm{C}$ were obtained under a $5.5 \mathrm{~V}$ bias, with heating response times between 100 and 200

1220 seconds. ${ }^{[217]}$ Finally, in a similar approach, Roul et al. reported AZO/Ag/AZO electrodes made

1221 by sputtering, on PET substrates. ${ }^{[218]}$ The structural, electrical and optical properties were

1222 evaluated as a function of Ag layer thickness. The best results were obtained for a thickness of

$12235 \mathrm{~nm}$, for which temperatures above $100{ }^{\circ} \mathrm{C}$ were obtained for a bias voltage of $10 \mathrm{~V} \cdot{ }^{[218]} \mathrm{In}$

1224 another case of a multilayer transparent electrodes, Kang et al. proposed the study of

1225 polymer/Ag/polymer and polymer/Ag/inorganic (ITO or $\mathrm{SiN}_{\mathrm{x}}$ ) tri-layer electrodes. ${ }^{[219]}$ The

1226 electrodes were produced by an R2R sputtering system, and the addition of a fluorocarbon

1227 polymer led to a higher flexibility, with the electrodes sustaining bending up to a radius of 3

$1228 \mathrm{~mm}$, with a sheet resistance of less than $5 \Omega / \mathrm{sq}$ and a transmittance of $68 \%$. The use of

1229 fluorocarbon polymer also yielded water-repelling electrodes, preventing wetting and removing

1230 contamination. THs based on fluorocarbon/Ag/SiN $\mathrm{x}_{\mathrm{x}}$ reached $180{ }^{\circ} \mathrm{C}$ for a bias voltage of 13

1231 V. ${ }^{[219]}$ Kim et al. have presented an asymmetric multilayer TH based on a GZO seed layer, an

1232 Ag metallic layer and a GZO optoelectronic control layer. ${ }^{[220]}$ The electrodes were deposited

1233 by sputtering at room temperature, and they showed an optimum sheet resistance of $5.4 \Omega$ /sq

1234 for a transmittance of $81.6 \%(50 \mathrm{~nm} \mathrm{GZO} / 12 \mathrm{~nm} \mathrm{Ag} / 50 \mathrm{~nm} \mathrm{GZO})$. The electrodes were

1235 deposited on glass substrates of different thicknesses, in order to evaluate the effect of heat loss

1236 through the substrate. The temperature reached on the film and the substrate, as well as the

1237 rising time, both depended on the substrate thickness. Heat loss due to conduction needs to be

1238 considered for substrates $\geq 3 \mathrm{~mm}$. A model is presented that allows the calculation of heating

1239 rates and deicing times. ${ }^{[220]}$ In a different approach, Im et al. proposed THs based of CNT sheets

1240 with granular metal (Pd). ${ }^{[221]}$ The addition of these metal particles boosted the heating efficiency

1241 by a factor of 3.6 , from 99.9 to $27.3{ }^{\circ} \mathrm{C} \mathrm{cm} / \mathrm{W}$. This improvement is attributed to electron-

1242 lattice interaction and heat loss suppression. Finally, Hudaya et al. reported THs based on FTO 


\section{WILEY-VCH}

1243 with scattered metal nanodots ( $\mathrm{Cr}, \mathrm{NiCr}$ and $\mathrm{Ni}$ ). The FTO layers were deposited by sputtering,

1244 while the metallic nanodots were deposited by electron cyclotron resonance CVD. ${ }^{[222]}$ The

1245 electrodes showed transmittance values over $85 \%$ and reached saturation temperatures over 80

$1246{ }^{\circ} \mathrm{C}$ for a bias voltage of $12 \mathrm{~V}$.

1247 These and other approaches, like $\mathrm{TiO}_{2} / \mathrm{Ag} / \mathrm{TiO}_{2}$ multilayers, have been explored in recent

1248 years. ${ }^{[223]}$ While only the examples described above have been evaluated as THs, it is expected

1249 that more electrodes based on the combination of different materials will also be evaluated for

1250 TH applications.

\subsection{Synthetic comparison between the different TH technologies}

1253 As previously described, there are different $\mathrm{TH}$ technologies which exhibit diverse

1254 characteristics. We have briefly reminded the main features for each technology, and will 1255 compare them in more detail in this section. TCOs are the most investigated/used TH 1256 technology in industrial devices, ${ }^{[10]}$ and were discussed in section 3.1 . They certainly are the 1257 most stable technology, at least in terms of electrical and thermal stability, but they are not 1258 compatible with flexible devices nor with solution-based fabrication processes. Moreover, due 1259 to indium scarcity, technologies using more abundant or cheaper materials, have been 1260 investigated. ${ }^{[21]}$ Carbon-based materials are mainly represented by carbon nanotubes and 1261 graphene, which have already been integrated in TH devices by several research teams (see 1262 section 3.2). ${ }^{[27,61,143]}$ Lately there has been a clear interest in metallic-based THs (as reported in 1263 section 3.3). MNW random networks have been well investigated these past years, in particular $1264 \mathrm{AgNW}^{[20,39]}$ and to a lesser extent CuNW. ${ }^{[48,66,169,224]}$ Interesting properties in terms of TH 1265 behavior have been reported with this technology, along with promising flexibility and cost1266 effective solution process fabrication. MNW-based THs appear compatible with large scale

1267 industrial fabrication such as roll-to-roll technology. ${ }^{[42,44,45]}$ In parallel, metallic mesh networks 1268 and grids also exhibit good physical properties and constitute efficient THs. ${ }^{[72,112,148]}$ However, 


\section{WILEY-VCH}

1269 the thermal and electrical stability of these metallic-based THs can be a severe issue. However,

1270 as shown by $\mathrm{Chen}$ et al., ${ }^{[56]} \mathrm{Cu}$ based grids made from a $\mathrm{Ag}$ seed layer and a subsequent

1271 electroplating of $\mathrm{Cu}$ imprinted microgrooves exhibit very good performances. Indeed, sheet

1272 resistance down to $0.03 \Omega / \mathrm{sq}$ associated with a transmittance of $86 \%$ was demonstrated.

1273 Furthermore these TH exhibit good stability. The possibility to fabricate them by roll-to-roll

1274 manufacturing appears promising for industrial integration. Metal nanofibers present also

1275 interesting physical properties in recent studies. ${ }^{[75,87]}$ Additionally, very recent efforts have led

1276 to drastically improve the properties of conductive polymers ${ }^{[49]}$, reaching the conductivity of

1277 state-of-the-art FTO layers (i.e. $\left.3 \cdot 10^{-4} \mathrm{~S} / \mathrm{cm}\right)^{[14]}$. By investigating different dopants for poly $(3,4-$

1278 ethylenedioxythiophene) (PEDOT)-based materials, Gueye et al. reported thin polymer films

1279 with a $R_{s h}$ of $57 \Omega /$ sq, a transparency of $87 \%$ and a very low haze factor (i.e. $<1 \%$ ). The use of

1280 these conductive polymers enables the fabrication of all-polymer-based THs with excellent

1281 performances ${ }^{[50]}$, with steady-state temperatures exceeding $100{ }^{\circ} \mathrm{C}$ when subjected to a $12 \mathrm{~V}$

1282 bias.

1283 As described in section 3.5, the association of the different aforementioned materials leads to

1284 nanocomposites and/or hybrids, constituting a very rich family of TH technologies that offer a

1285 large panel of properties/performances with clearly enhanced electrical and thermal stabilities.

1286 The ongoing efforts by the community to fabricate, better understand and optimize THs is

1287 notably driven by the growing desire for low-cost, more stable and efficient THs. These efforts

1288 can efficiently address several challenges for many applications, the latter being described

1289 below in section 4.

1290 The comparison of these different technologies is represented in Figure 15, with several criteria

1291 being considered. It is worth mentioning that these criteria and the marks associated to each

1292 criterion and for each TH technology are subject to discussion, therefore only general trends

1293 should be considered. Depending on experimental TH synthesis and usage, a range of figures

1294 of merit with minimum and maximum values are proposed. Exceptions ruling out these trends 


\section{WILEY-VCH}

1295 do exist. The flexibility criterion refers to the capacity of a TH technology to withstand

1296 bendability or, for some applications, stretchability tests. TCOs are generally not flexible since

1297 oxides belong to the ceramic family, which are brittle materials (see Figure 7d), while other TH

1298 technologies are highly flexible. This is the case of all emerging TH technologies. Another

1299 critical issue is thermal stability: TCOs are very stable compared to other TH technologies. Thin

1300 oxide layers are even used as coatings for other TH technologies, forming efficient and stable

1301 nanocomposite THs. Conductive polymers are the least thermally stable among all $\mathrm{TH}$

1302 technologies due to their organic nature. Nevertheless high temperatures (above $100^{\circ} \mathrm{C}$ ) can be

1303 maintained for a long time, which is sufficient for most applications. The cost of a TH

1304 technology depends on several parameters, including the industrial production chain. The

1305 learning curves generally show a power law dependency between the cost of a device and the

1306 total cumulative production amount. Spatial uniformity is an application-dependent criterion.

1307 Homogeneous and continuous thin layers (such as TCOs, conductive polymers or some

1308 nanocomposites) are uniform by nature, while networks of carbon nanotubes or metallic

1309 nanowires, tiled graphene-based sheets or metallic grids can appear non-homogeneous, as

1310 demonstrated by the appearance of hot spots in the worst case. ${ }^{[187]}$ The use of nanocomposites

1311 combining both continuous and discontinuous THs can prevent this non-uniformity. Haacke's

1312 figure of merit (FoM) was used $\left(\operatorname{Tr}^{10} / R_{s h}\right)$ : generally speaking, apart from graphene and CNT

1313 networks this FoM has rather high values for all TH technologies. Finally, the technological

1314 readiness level (TRL) criterion was used. The highest TRL value belongs to the TCO family,

1315 which has been investigated and used in industrial devices for several decades. The lowest TRL

1316 value is for the conductive polymers, for which the drastic progresses of conductivity making

1317 them very promising are only recent. ${ }^{[49,50]}$ 


\section{WILEY-VCH}
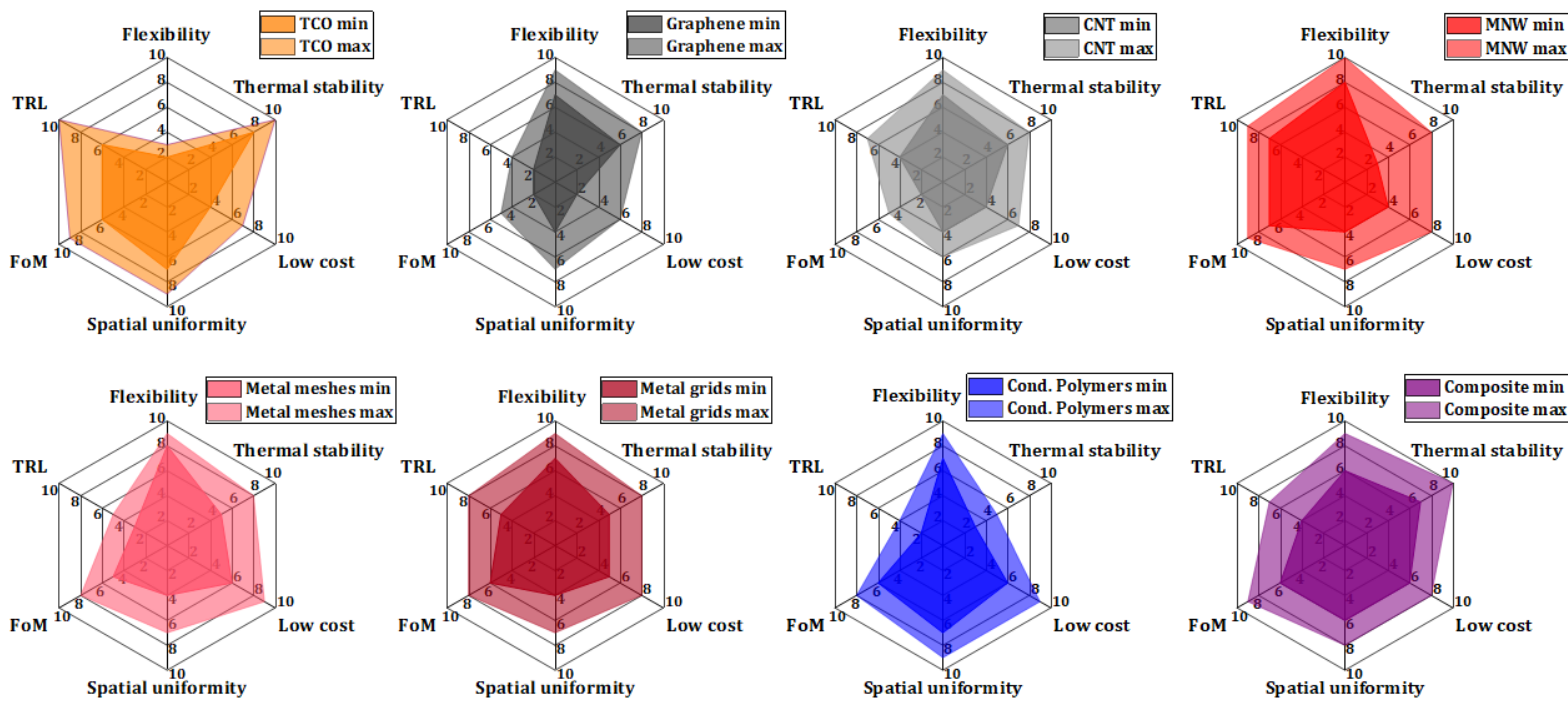

Figure 15. Comparison of THs fabricated with the different technologies. This figure shows the general trends with six criteria (clockwise from the top): flexibility, thermal stability, low cost (of both material and process), spatial uniformity, figure of merit (FoM) and technological readiness level (TRL). Depending on the synthesis and usage of experimental THs, a range of figures of merit with minimum and maximum values is proposed. The marks for all criteria for each $\mathrm{TH}$ technology are only general indications, since the actual marks are application-dependent.

Other characteristics could also be of interest. This is the case for the haze factor, which should be low (typically below 2 or $3 \%$ ) when a TH is placed on windscreens, visors or displays to ensure eye comfort (preventing blurriness). The haze factor is typically low for TCOs, while it can vary drastically for other $\mathrm{TH}$ technologies. For instance, small $\mathrm{MNW}$ diameters are preferred to decrease the haze factor ${ }^{[96]}$ of MNW-based THs. Recently, Ji et al. reported a study of ultra-long copper nanofibers covered with a shell of carbon black, which can provide a highclarity view (i.e. with low haziness) associated to a transparency of $91 \%$ and a sheet resistance of $0.8 \Omega /$ sq. ${ }^{[87]}$ This once again shows that nanocomposite THs can exhibit excellent properties through efficient combinations of key assets from different $\mathrm{TH}$ technologies.

\section{Integration of transparent heaters in devices:}

1337 As described above, THs concern numerous applications. TH fabrication is generally carried

1338 out with the goal of implementation in a specific device and for a particular application. As 1339 such, active material choice and device design must be considered according to specifications 


\section{WILEY-VCH}

1340 related to the operational use of the device. After pointing out some issues related to $\mathrm{TH}$

1341 fabrication (part 4.1), we will describe examples of technical uses. The first one deals with THs

1342 for deicing, defrosting or defogging systems (4.2). Then we show how THs can be relevant for

1343 thermochromic devices (4.3), medical applications (4.4) and other niche applications (4.5).

\subsection{Integration of THs within devices: generalities and potential issues}

Even though the intrinsic performances of the materials developed for THs are generally very good, their integration into functional devices can encounter some difficulties. Integration will depend on the nature of the active and support materials, and in general on the environment close to the heating film. In some cases, adhesion can be an issue if the deposited active layer does not stick well to the substrate. This can occur with metallic nanowires, which usually show

1351 limited adhesion and can be removed by a simple finger sweep. Layers of graphene- or PEDOTbased materials can be very thin (i.e. less than $20 \mathrm{~nm}$ ) and thus very fragile and sensitive to mechanical stress. An encapsulating layer needs to be deposited on top of these materials to ensure a good adhesion to the surface, to avoid mechanical damage while preventing the release of the nanomaterials towards end-users. This encapsulation can be carried out through different techniques, like wet processes for transparent polymers, physical deposition of TCOs by atomic layer deposition, lamination with thin glass sheets or transparent adhesive films. As mentioned earlier, haziness must be finely controlled during the fabrication process since the haze value can vary significantly for some materials. Low haze values are mandatory for

1360 TH applications for windscreens or displays, whereas haziness may not be critical for other 1361 applications. This means that the fine control of the material itself (e.g. metallic nanowire 1362 diameter $^{[97]}$ or layer thickness of carbon-based material) is crucial to reach the desired 1363 specifications and homogeneity on large surfaces. Depending of the TH application, tints 1364 induced by the materials should be considered (from a blue- or greenish hue for conductive 


\section{WILEY-VCH}

1365 polymers, blackish for CNTs, metallic greyish or a slight orange color for silver and copper

1366 nanowires, respectively, to yellowish with iridescence for TCOs).

1367 Despite their importance, electrical contacts are seldom mentioned in the reported works

1368 dealing with TH integration. The fabrication of good and reliable electrical contacts between

1369 the active layer and the voltage generator is usually not straightforward. Silver-based inks or

1370 pastes are often used for lab-scale demonstrations, but these materials are not always stable in

1371 the long term, and their homogeneous deposition can be tricky. Many other techniques and

1372 materials can be envisaged, like vacuum-deposited metals (gold, silver, copper, nickel, etc.),

1373 metallic ribbons or wires (usually copper-based materials), liquid metals (e.g. eutectic gallium-

1374 indium), or even materials with similar chemical natures to those used in THs, taking into

1375 account that these contact electrodes must be much less resistive than the active layers of the

$1376 \mathrm{TH}$ in order to avoid hotspots and Joule heating at unexpected locations.

1377 Beyond the nature of the electrical contacts, the geometry and patterns of contact electrodes are

1378 very important. They will define the heating zones. Depending on their design and voltage

1379 input, specific areas can be heated at different areal power densities. In a basic case study with

1380 two opposite electrodes on a square $\mathrm{TH}$, the distance between the electrodes must be well

1381 designed with respect to the available bias supply. Indeed, the voltage must be increased when

1382 the distance between the electrodes is increased to keep good heating properties (generally at

1383 least a few hundreds of $\mathrm{W} / \mathrm{m}^{2}$ ). If this distance increases too much, then high voltages must be

1384 used, which can seriously damage the active materials.

1385 TH production should also consider the end-user application requirements. For highly flexible

1386 substrates for example, TCOs will not be suitable and polymer materials or metallic nanowires

1387 will be preferred. This choice of active material will also define the relevant and available

1388 deposition techniques, which are active material-dependent. For large area deposition

1389 techniques, special care should be taken to ensure homogeneous deposition, and ideally online

1390 analysis techniques should be implemented to check deposition uniformity. 


\section{WILEY-VCH}

1392

1393

1394

1395

1396

1397

1398

1399

1400

1401

1402

1403

1404

1405

1406

1407

1408

1409

1410

1411

1412

1413 1414 film heater at the surface of the screen is an easy and lightweight method to heat the display

1415 and avoid degradation linked to low temperatures.

\subsection{Deicing, defrosting and defogging}

The first application of THs was to avoid condensation in aircraft windshields to increase visibility in warplanes during military actions. At that time, the TCM used was tin oxide, but in modern airplanes it has been replaced by ITO, which is more conductive and thus allows a lower operating voltage $(24 \mathrm{~V}) \cdot{ }^{[2]}$

The heat produced by THs can also be used for defogging or defrosting applications. Defogging of car headlights is a recent need. Indeed, since the invention of cars, headlights have been based on incandescent bulbs, which inherently produce heat, preventing headlight condensation when they are on. Light-emitting diodes (LEDs) are now gradually replacing conventional incandescent lamps, because of their reduced energy consumption. ${ }^{[225]}$ Despite this obvious advantage, this new kind of headlight does not produce enough heat to avoid condensation or frosting at the surface of the headlights or inside the headlight housing. This situation creates a new risk, since fog or frost on the headlights can change light refraction and increase light diffusion, which degrades road illumination and thus visibility for the driver.

There is a market for defogging devices based on THs in food distribution, with refrigerated showcases using transparent windows ${ }^{[2]}$. Indeed, when products are refrigerated, there is a risk of condensation because of the temperature difference between the two faces of the case. Consumers not being able to see products behind the windows can be detrimental to the store; THs offer an efficient solution to this problem, and patents linked to this topic have existed since the 90s, with ITO technologies. ${ }^{[226]}$

LCD panels are used for outdoor applications. A limitation of LCD displays is liquid crystals freezing and electronic performance degradation at low temperatures ${ }^{[227,228,61]}$. An ultra-thin 


\section{WILEY-VCH}

1416 For car windows (windshield and side windows) and external side mirrors, or even for

1417 motorcycle helmet visors, the operating voltage should ideally not exceed $12 \mathrm{~V}$, which is the

1418 current standard voltage for cars and motorcycles. This is one of the reasons for the demand for

1419 new transparent coatings with lower resistances and similar transparency. The development of

1420 the $48 \mathrm{~V}$ vehicle will allow, in the near future, to reduce the pressure on the power management

1421 of large surface THs and will open up new prospects. Other reasons behind the demand for new

1422 transparent coatings concern flexibility and the ability to coat curved surfaces. As previously

1423 mentioned, some technologies meet these requirements, especially AgNWs, CNTs, graphene

1424 and conducting polymers.

1425 Many demo products have been fabricated, like defoggers/defrosters for car windows or side

1426 mirrors (Figure 16a,b), made of patterned $\mathrm{AgNWs},{ }^{[229,230]} \mathrm{AgNFs}^{[231]} \mathrm{Cu}$ grids ${ }^{[56]}$ or Ni/Ag

1427 microgrids. ${ }^{[195]}$ A demo motorcycle visor (Figure 16c) dip-coated with PEDOT:PSS-EG was

1428 fabricated and can be defrosted in a few seconds with a low voltage. ${ }^{[50]} \mathrm{Zn}-\mathrm{Sn}$ -

1429 oxide(ZTO)/Ag/ZTO was also demonstrated to be of interest for small area defoggers (Figure

$143016 \mathrm{~d}$ ), as well as for larger areas like the windshield of a commercial car. ${ }^{[232]}$ Since these systems

1431 require low voltages, even sport glasses/ski masks could integrate these new technologies in

1432 the coming years.

1433 


\section{WILEY-VCH}

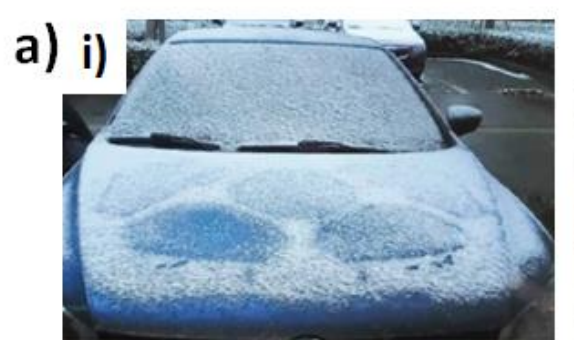

b) i)

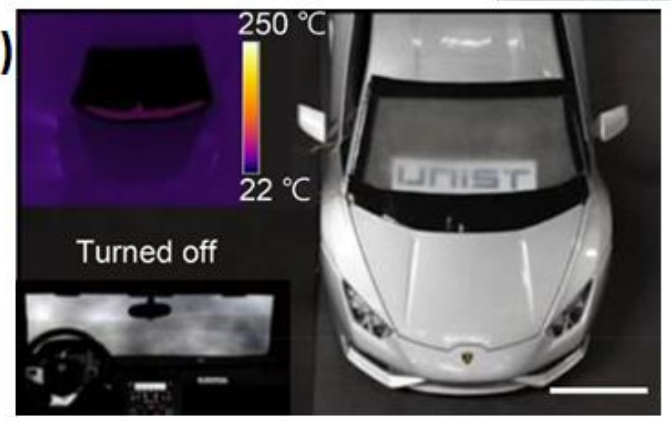

c) i)

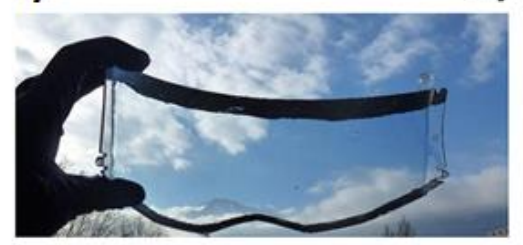

ii) Before

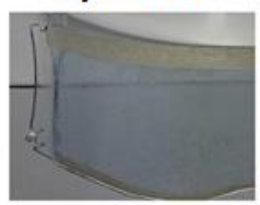

\section{iii) After}

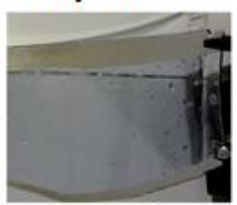

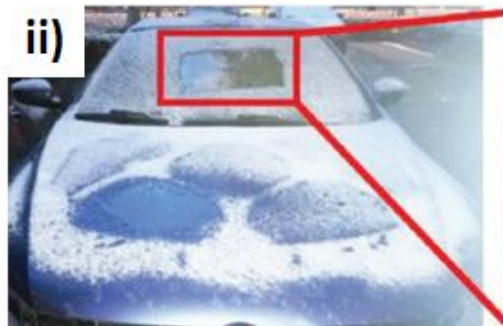

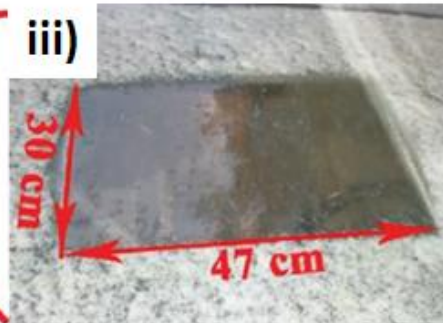

ii)

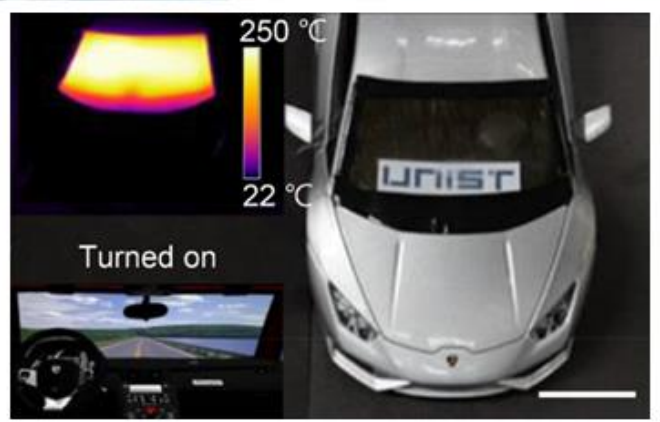

d) i) Before fogging

ii) After fogging

ii) After de-fogging
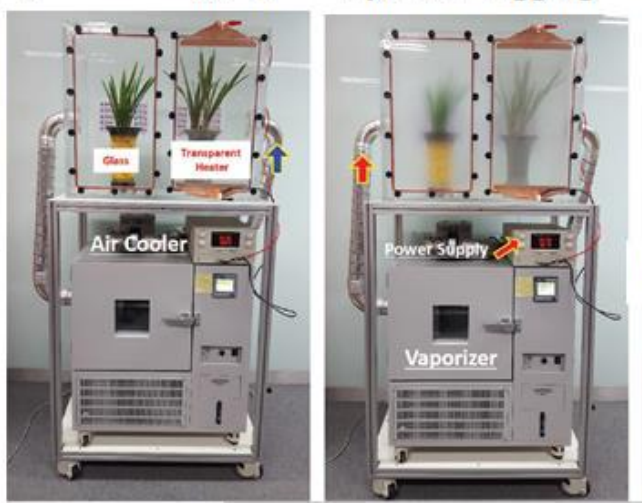

iv) Window Temp.

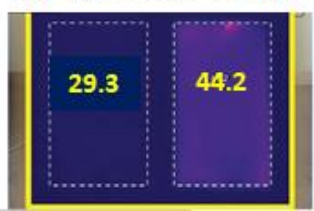

1435

1436

1437

1438

1439

1440

1441

1442

1443

1444

1445

1446

1447

1448

1449

1450

1451

1452

Figure 16. Defrosting and defogging transparent heater applications. a) A car windshield i) before and ii)-iii) after defrosting by applying $20 \mathrm{~V}$ for 5 min to a large-size $\mathrm{Cu}$ metal-mesh $\left(30 \mathrm{x} 47 \mathrm{~cm}^{2}\right)$. Reproduced with permission. ${ }^{[56]}$ Copyright 2019, John Wiley and Sons. b) Photographs of the $\mathrm{Ag} / \mathrm{Ag}_{2} \mathrm{O}-$ $\mathrm{NF} /($ index matching layer)IML heater-integrated automobile windshield at i) turn-off and ii) turn-on modes. The applied dc bias was $6 \mathrm{~V}$. The insets show a driver's view through the windshield (lower) and an image captured with an IR camera displaying the temperature distribution of the windshield (upper). Reproduced with permission. ${ }^{[231]}$ Copyright 2018, American Chemical Society. c) i) a motorcycle visor with a PEDOT:PSS transparent heater, ii) iced in a freezer at $-26{ }^{\circ} \mathrm{C}$ and iii) after defrosting by the TH. Reproduced with permission. ${ }^{[50]}$ Copyright 2017, American Chemical Society. d) Pictures describing the quality test of a Zn-Sn-oxide(ZTO)/Ag/ZTO transparent heater $\left(52 \times 31 \mathrm{~cm}^{2}\right)$; i) general setting of the test with a normal glass and transparent heater glass placed in front of each orchid before running a test, ii) fog was generated on both windows using a moisturized hot air flow, iii) defogging was carried out on the heater glass for $50 \mathrm{~s}$ at $12 \mathrm{~V}$, and (iv) temperature profile of the two glasses during defogging. Reproduced with permission. ${ }^{[232]}$ Copyright 2019, John Wiley and Sons.

\subsection{Thermochromic applications}

The alteration of the color or transmittance of thermochromic materials induced by changes in

temperature can be used in a large variety of applications such as smart windows and green 


\section{WILEY-VCH}

1453 buildings, aerospace and military, textile and food packaging. ${ }^{[233]}$ Thermochromic smart

1454 windows are attractive because they are visibly transparent and can intelligently control the

1455 amount of solar heat (mainly in the near-infrared region) in response to changes in ambient

1456 temperature. ${ }^{[234]}$ Thermochromic applications are attracting more and more scientific and

1457 industrial interests, with efforts being devoted to their successful integration into modern

1458 devices. The research discussed in this part shows the Joule effect-based TH influence on

1459 thermochromic performance, the associated issues like layer adhesion, and the integration in

1460 devices. Vanadium oxide $\mathrm{VO}_{2}$ materials are often presented, because they exhibit the greatest

1461 potential for thermochromic devices and energy-efficient systems ${ }^{[235]}$ thanks to their electronic

1462 structure modification from semi-conducting to metallic behavior when the temperature

1463 exceeds $68^{\circ} \mathrm{C}$. Emerging TCMs like CNTs ${ }^{[236]}$, graphene ${ }^{[53]}, \operatorname{AgNWs}^{[109,200,237,238]}$, conductive

1464 polymers ${ }^{[50]}$ and hybrids ${ }^{[102,203]}$ have been investigated as the heating elements.

1465 Liu et al. ${ }^{[236]}$ developed a CNT-based flexible microheater on PET for local heating into a

1466 thermochromic display, and tuned the thickness of the materials in order to optimize the

1467 thermochromic performance. A first approach was to decrease substrate thickness in order to

1468 obtain a good trade-off between mechanical robustness and thermal response. The thermal

1469 response of CNT-based films from room temperature to $100{ }^{\circ} \mathrm{C}$ was reduced to a few seconds

1470 thanks to the use of a $70 \mu \mathrm{m}$ thick PET layer and with lower CNT coverage. ${ }^{[236]}$ Indeed, heat

1471 dissipation increases inversely with CNT coverage. Finally, a thermochromic display coupled

1472 to a driving circuit was made, and Chinese characters were displayed thanks to local heating by

1473 the CNT microheater on PET.

1474 In parallel, Kim et al. ${ }^{[53]}$ reported interesting progress related to the fabrication of flexible

$1475 \mathrm{VO}_{2} /$ Graphene-based thermochromic films for energy-saving windows. Graphene acts as a very

1476 thin (i.e. atomic film), flexible but robust substrate for the formation of stoichiometric $\mathrm{VO}_{2}$

1477 crystals. The transfer of the graphene-supported $\mathrm{VO}_{2}$ onto a plastic substrate enables the 


\section{WILEY-VCH}

1478 formation of a flexible thermochromic film which shows a decrease of in-house temperature

1479 under infrared irradiation in a mock-up house.

1480 In the case of AgNW-based THs, Li et al. ${ }^{[237]}$ studied the influence of AgNW networks on the

$1481 \mathrm{VO}_{2}$ nanoparticle-based thermochromic performance. The infrared response of this

$1482 \mathrm{VO}_{2} / \mathrm{AgNW}$-based device can be controlled thanks to the applied voltage, and the dependence

1483 is shown to be highly stable and reversible. Figure 17a shows the square-like shape of the

1484 dynamic infrared response to the pulse voltage. The response of the $\mathrm{VO}_{2} / \mathrm{AgNW}$-based device

1485 exhibits a step variation. The transmittance in the infrared region can therefore quickly be

1486 altered when applying either rising or falling input voltage.

1487 Huang et al. ${ }^{[238]}$ fabricated a flexible AgNW/PI TH with faster and higher heating temperatures

1488 at lower power consumption compared to TCO heaters. The flexible and stable TH was

1489 successfully used in a thermochromic device. The obtained AgNW/PI-based TH exhibited a

1490 heating temperature of $96^{\circ} \mathrm{C}$ under a bias of $6 \mathrm{~V}$, and a response time of 40 seconds. ${ }^{[238]}$

1491 Recently, Huang et al. ${ }^{[200]}$ fabricated a large-area flexible, transparent thermochromic window

1492 based on AgNW nylon-meshes. This thermochromic textile could be produced cheap and fast,

1493 i.e. 20 minutes for $7.5 \mathrm{~m}^{2}$ and a cost of $\$ 15.03$.

1494 Another interesting and recent study presented by Kim et al. ${ }^{[109]}$ used AgNW-based THs as a

1495 color-shifting anisotropic soft actuator with a very large bending curvature of $2.5 \mathrm{~cm}^{-1}$ at a low

1496 temperature of $40{ }^{\circ} \mathrm{C}$. The transient behavior of the curvature and temperature of the color

1497 changing actuators based on AgNW Joule heating is shown in Figure 17b as well as digital

1498 camera images of the color of the actuator. The devices have demonstrated a superior long-term

1499 stability for more than 10,000 operating cycles. Shi et al. ${ }^{[203]}$ demonstrated a

$1500 \mathrm{SiO}_{2} / \mathrm{AgNW} /$ colorless PI composite film heater with an improved transparency of $3.5 \%$ due to

1501 the antireflective $\mathrm{SiO}_{2}$ coating. The haze of both bare and $\mathrm{SiO}_{2}$-coated $\mathrm{AgNW} / \mathrm{cPI}$ films are the

1502 same, as well as their heating performance. The composite shows good mechanical properties,

1503 and was tested in a thermochromic device with a purple to blue color change after $10 \mathrm{~s}$ of 


\section{WILEY-VCH}

1504 applied voltage. Kim et al. ${ }^{[102]}$ fabricated a CNT/AgNW hybrid TH with reduced haze and

1505

1506

1507

1508

a)

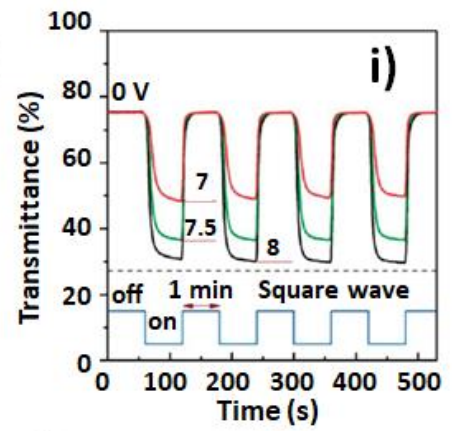

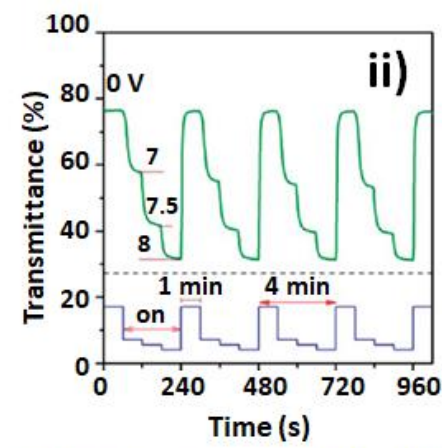

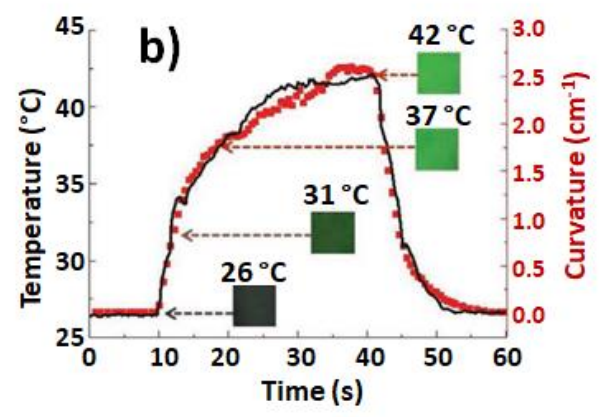

c) i)
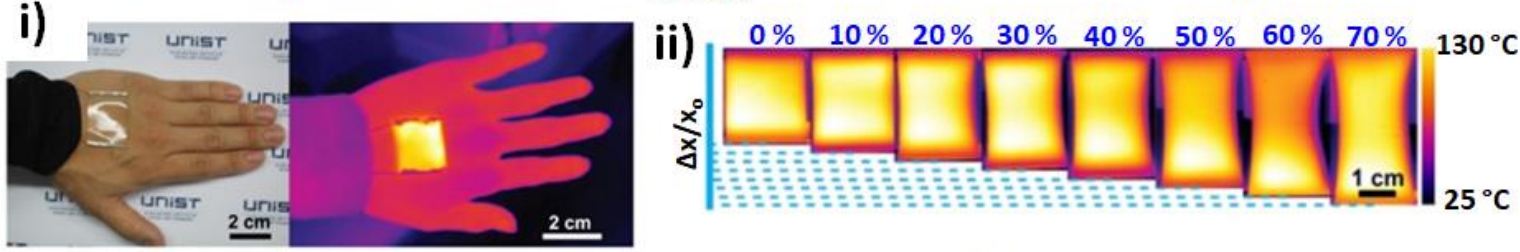

d)
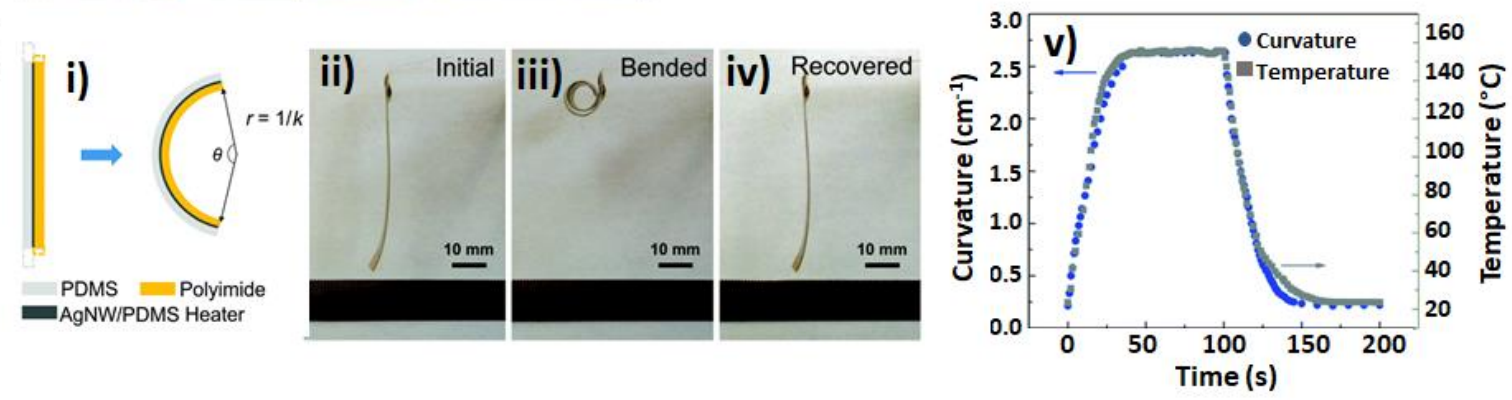

Figure 17. Thermochromic, medical and other transparent heater applications. a) Infrared response at $1.5 \mathrm{~mm}$ of a $\mathrm{VO}_{2} \mathrm{NP}$-electrothermochromic film device based on AgNWs upon input of a pulse voltage with i) a square wave and ii) a square wave with a step variation. The dashed line denotes the lowest transmittance at a constant voltage of $8 \mathrm{~V}$. The pulse wave pattern and its application method are shown below the dashed line, along with the wave parameters. Reproduced with permission. ${ }^{[237]}$ Copyright 2014, Royal Society of Chemistry. b) The transient behavior of the curvature and temperature of a colorshifting anisotropic soft actuator based on an AgNW network TH. Insets are digital camera images of the actuator's color changes with respect to temperature. Reproduced with permission. ${ }^{[109]}$ Copyright 2018, John Wiley and Sons. c) Transparent and wearable heaters using nanotrough network of CuZr metallic glasses; i) photograph and IR image of the heater attached to a human hand. The black strap is the custom-made electronic band for a power supply and temperature controller. ii) IR images of the $\mathrm{CuZr}$ nanotrough heater under various tensile strains. Reproduced with permission. ${ }^{[70]}$ Copyright 2016 , American Chemical Society. d) Bending and heating performance of the PI/AgNW/PDMS bimorph actuator; i) schematic of the electrothermal bimorph actuation mechanism. $\mathrm{k}$ : bending curvature; $\mathrm{r}$ : radius of curvature, ii) the initial state, iii) the bent state with the maximum curvature and iv) the recovered state of the U-shaped actuator. One end of the actuator is fixed and the other end can bend freely and reversibly under an applied DC voltage of $4.5 \mathrm{~V}$. v) Curvature and temperature of the actuator as a function of time. Reproduced with permission. ${ }^{[239]}$ Copyright 2017, Royal Society of Chemistry. 


\section{WILEY-VCH}

\subsection{Transparent heaters for Medical applications}

1530 This last decade has seen the emergence of new flexible and stretchable electronics to fabricate

1531 sensors and actuators with properties similar to skin (namely deformation of up to $15 \%$ and

1532 elastic modulus between $10 \mathrm{kPa}$ and a few hundred $\left.\mathrm{kPa}^{[240]}\right)$. These kinds of devices can be

1533 found under several names, namely "electronic skin", “e-skin”, “epidermal electronics“ and

1534 "electronic tattoos". ${ }^{[241]}$ They present the advantages of being skin-mountable and thus relevant

1535 for continuous health monitoring, drug delivery, thermotherapy, human motion detection,

1536 human-machine interfaces and soft robotics. ${ }^{[241-243]}$

1537 Since 2013 , Webb et al. ${ }^{[244,245]}$ have worked on the continuous thermal characterization of the

1538 skin by using ultrathin arrays of sensors and heaters. Combining sensing and heating allows to

1539 monitor physical and chemical parameters (like body temperature, glycemia, blood pressure

1540 and oxygen, elastic modulus of the $\operatorname{skin}^{[241]}$ ) and potentially immediately diagnosing and

1541 delivering a medical treatment by cutaneous contact, keeping in mind that skin permeability or

1542 healing can be improved by local heating ${ }^{[246]}$.

1543 Epidermal drug delivery systems are attractive because of the reduced side effects of topical

1544 administration. Bagherifard et al. ${ }^{[243]}$ used flexible heaters in dermal patches to control the

1545 release rates of drugs encapsulated into a thermo-responsive hydrogel (N-Isopropylacrylamide,

1546 NIPAM).

1547 Currently, sensor heaters are generally made of a bilayer of chromium and gold ${ }^{[243-245]}$, which

1548 are not transparent. Nevertheless, the nanometric thickness of the circuit results in a quasi-

1549 transparent device and highlights this line of research.

1550 The most important application of electronic skins is thermotherapy, which is a new but

1551 expanding field. Indeed, heating pads are commonly used for a wide range of medical functions,

1552 like relieving pain ${ }^{[247,248]}$, preventing inflammation and improving blood circulation. ${ }^{[21]}$ 


\section{WILEY-VCH}

1553 Aside from pain relief, beneficial effects of a local heating treatment can be explained by the

1554 vasodilation, allowing blood vessels to supply more oxygen and nutrients to the surrounding

1555 area. Heating also prevents chronic inflammation by expelling inflammatory exudates. ${ }^{\text {[249] }}$

1556 Moreover, heating pads can be useful in physiotherapy to help tendons, ligaments and muscles

1557 gain flexibility. ${ }^{[250]}$ The two currently available technical solutions for thermotherapy are heat

1558 packs and wraps. ${ }^{[250,251]}$ Heat packs are bulky and heavy and must be pre-heated in water or in

1559 a micro-wave, often compromising the fine control of the temperature. Heating wraps allow a

1560 better control of the temperature thanks to the Joule heating of resistors, but are usually rigid

1561 and heavy, which limits their wearability and their use in hospitals. ${ }^{[23]}$

1562 New lightweight and autonomous devices combining a controlled temperature, fast response

1563 and high transparency are gaining interest. Using THs for thermotherapy pads would allow to

1564 see the heated parts of the body, potentially healing wounds, and to adjust more efficiently the

1565 treatment. Different techniques are under development to meet these requirements. The average

1566 values of temperature/voltage should be around $40-50{ }^{\circ} \mathrm{C} / 3-5 \mathrm{~V}$, to avoid burning the skin and

1567 to operate the devices with common batteries. Two methods are mostly studied: patterned

1568 nanocomposites and hybrid films with a network of metallic conductors and an elastomeric

1569 substrate.

1570 In 2015, Choi et al. ${ }^{[23]}$ developed a patterned nanocomposite made of AgNWs and a

1571 thermoplastic elastomer (styrene-butadiene-styrene, SBS). In order to obtain a highly

1572 conductive nanocomposite, a ligand-exchange reaction was carried out to homogeneously

1573 incorporate the AgNWs within the elastomeric matrix. This serpentine mesh-structure allows

1574 stable heating $\left(+40{ }^{\circ} \mathrm{C}, 1 \mathrm{~V}\right)$ while being stretched $(17 \%$ and $26 \%$ for extension or flexion of

1575 the wrist and the knee, respectively). The resistance remains stable after 5000 cycles at $30 \%$

1576 strain and $48 \mathrm{~h}$ under UV exposure. Unfortunately, the device transparency value was not

1577 reported. 


\section{WILEY-VCH}

1578 Other techniques being developed use conductive networks based on MNWs or metallic

1579 glasses, coated on elastomeric substrates. CuZr metallic glasses on PDMS display excellent 1580 optoelectronic properties, with a resistance of $4 \Omega$ /sq for $90 \%$ transparency, combined with a 1581 high stretchability (only $30 \%$ change in resistance for $70 \%$ strain, Figure $17 \mathrm{c}$ )). ${ }^{[70]}$ Moreover, 1582 a temperature of $60{ }^{\circ} \mathrm{C}$ can be reached for a bias of only $3 \mathrm{~V}$. In terms of stability it is 1583 demonstrated that the heating performance of the film is stable for 10 days under relatively 1584 harsh conditions $\left(85{ }^{\circ} \mathrm{C}\right.$ for $\left.85 \% \mathrm{RH}\right)$. CuNW-based hybrids also present good performances, with $96 \Omega /$ sq for $91 \%$ transparency, using a poly(methyl methacrylate) (PMMA) coating. ${ }^{\text {[252] }}$

1586 To obtain a stretchable device, the elastomer Ecoflex ${ }^{\circledR}$ (blend of cellulose, lignin, poly(lactic 1587 acid), poly(hydroxy alkanoate) and starch) was used as a replacement for PMMA, and enabled 1588 a stretching of $80 \%$ with a constant DC bias of $1.5 \mathrm{~V}$. Under these conditions, however, the 1589 maximal reported temperature reached only $30{ }^{\circ} \mathrm{C}$. If the stretching is kept low, a temperature 1590 of $50^{\circ} \mathrm{C}$ can be reached while applying $3 \mathrm{~V}$, which is sufficient for thermotherapy. Percolated 1591 AgNW networks deposited on PDMS and encapsulated with PVA were shown to have adequate 1592 properties for thermotherapy pads, with high transparency $(90 \%)$, low sheet resistance $(20 \Omega / \mathrm{sq}$ 1593 and a low operating voltage $\left(3.5 \mathrm{~V}\right.$ to reach $\left.45^{\circ} \mathrm{C}\right) .{ }^{[121]}$ Stability under severe thermal $\left(80{ }^{\circ} \mathrm{C}\right)$ 1594 and humidity $\left(80 \% \mathrm{RH}, 45^{\circ} \mathrm{C}\right)$ conditions was demonstrated for an extended period of 6 months, 1595 in addition to mechanical stability over 10,000 bending cycles ( $1 \mathrm{~mm}$ radius).

1596 Recently, the impact of skin surface heating has been evaluated thanks to tests on porcine skin, 1597 to evaluate the efficacy of thermotherapy. A $12{ }^{\circ} \mathrm{C}$ temperature raise of the subcutaneous tissue 1598 (5 mm beneath the skin surface) was measured. ${ }^{[190]}$

1599 Stretchable TCMs demonstrate promising potential for thermotherapy. The stretchability of 1600 THs for thermotherapy is an added value. Many research projects on these materials have 1601 emerged in recent years, often in strong relationship with the development of flexible and 1602 transparent electronics. ${ }^{[253]}$ Based on the growing interest in wearable devices and the wide 


\section{WILEY-VCH}

1603 range of medical applications, there is no doubt that the research on this topic will gain further

1604 momentum.

1605

1606

\subsection{Other niche applications}

1607 Besides the main applications previously described, THs are clearly interesting for other niche

1608 fields like actuators, gas sensors or refractive index tuning. Actuators are devices which are

1609 able to convert different types of energy, such as $\operatorname{light}^{[254]}$, electric fields ${ }^{[255]}$, magnetic

1610 fields ${ }^{[256]}$, pneumatic pressure ${ }^{[257]}$ or thermal energy, into mechanical energy. Among them,

1611 electrothermal actuators are based on either the thermal expansion of a single material or on the

1612 difference in thermal expansions between two materials. Their competitive advantages over

1613 other actuators are their low actuation voltage, as well as their lightweight and electrolyte-free

1614 design. Zhu et al. demonstrated the use of graphene in bimorph actuators. ${ }^{[258]}$ Graphene

1615 contracts upon heating due to a negative thermal expansion coefficient, which is opposite to the

1616 common behavior of nearly all materials. This asymmetric thermomechanical response leads to

1617 a large bimorph actuation. More recently, transparent electrothermal actuators using CNT-

1618 based THs ${ }^{[259,260]}$ and AgNW-based THs ${ }^{[109,239]}$ have been reported. Yao et al. demonstrated a

1619 remarkable $2.6 \mathrm{~cm}^{-1}$ curvature at a very low actuation voltage of $0.2 \mathrm{~V} / \mathrm{sq}(4.5 \mathrm{~V})$ in

1620 electrothermal actuators using AgNW THs (Figure 17d). ${ }^{[239]}$

1621 Several studies mention the use of thermal stimuli to address different functions. Zhang et al.

1622 developed a transparency-switchable electrothermal actuator using aligned CNT-based

1623 THs. ${ }^{[260]}$ In this paper, the authors used a paraffin wax/polydimethylsiloxane composite. At

1624 room temperature, the composite is opaque due to the light scattering induced by the paraffin

1625 wax crystallites. Upon heating, actuation occurs simultaneously with wax melting, which

1626 results in a dramatic increase in transmittance. Kim et al. demonstrated the use of MNW-based

1627 THs for simultaneous actuation and thermochromic color change. ${ }^{[109]}$ 


\section{WILEY-VCH}

1628 Transparency becomes an important feature for gas sensors. Since heating gas sensors can

1629 improve their performances, the use of Joule heating with THs was reported to improve 1630 sensitivity and/or recovery time. ${ }^{[62,261]}$ Choi et al. developed graphene-based gas sensors, in

1631 which graphene was used for both sensing and heating. ${ }^{[62]}$ They reduced the recovery time of

1632 the $\mathrm{NO}_{2}$ sensor by two orders of magnitude by heating the system up to $250^{\circ} \mathrm{C}$. Similarly, Walia 1633 et al. improved the response time (from 41 to $13 \mathrm{~s}$ ) and recovery time (from 112 to $35 \mathrm{~s}$ ) at $1 \%$

$1634 \mathrm{H}_{2}$, with a moderate Joule heating at $75^{\circ} \mathrm{C}$ of the transparent conducting palladium network 1635 used for sensing. ${ }^{[261]}$

1636 In another niche application, Heo et al. demonstrated the use of ITO-based THs for the

1637 development of lenticular lenses with a thermally tunable focus, using liquid crystals. ${ }^{[262]}$ The

1638 temperature can alter the birefringence of a liquid crystal-based lens by changing the refractive

1639 index between the active component and the polymer layer. Increasing the temperature from 25

$1640{ }^{\circ} \mathrm{C}$ to $55{ }^{\circ} \mathrm{C}$ altered the focal length from $5.5 \mathrm{~mm}$ to $8.5 \mathrm{~mm}$. These lenticular lenses have

1641 potential in optical devices like phone cameras.

1642 Finally Won et al. ${ }^{[263]}$ showed an original concept of stretchable kirigami TE consisting of

1643 ultrathin and flexible AgNWs/colorless polyimide cPI composites with laser patterned kirigami

1644 structure. A coating thin gold layer enables biocompatibility and enhances electrical stability.

1645 The laser patterning technique provides digital and rapid process (without patterning masks)

1646 allowing to design kirigami structures. ${ }^{[263]}$ This leads to achieve tunable stretchability, up to

1647 strain of $400 \%$, which extends the scope of applications. Such a soft, thin and highly stretchable

1648 features can pave ways for multifunctional transparent and wearable electronic skin 1649 applications. 


\section{WILEY-VCH}

16515 Prospects, future challenges and conclusive remarks

1652 Since the first use of THs approximately eighty years ago, tremendous progress has been made

1653 in this field. TCOs were the first materials developed and produced at the industrial scale to

1654 fabricate efficient THs. These materials have been highly improved over the past decades, and

1655 now reach outstanding properties, in particular thanks to an excellent transparency-conductivity

1656 trade-off. Although TCOs have been the materials of choice until now, new scientific

1657 breakthroughs and new market needs have opened the way for innovative developments,

1658 notably based on nano-enabled technologies. The driving forces to search for (improved)

1659 substitutes to TCOs not only rely on cost issues, but also on geostrategic considerations, which

1660 can be a game-changer, and on the technological evolution of devices. Many devices will

1661 require flexibility and stretchability (displays, PV cells, touch screens...), which requires new

1662 performances and abilities. The development of these transparent materials could offer an

1663 additional value to existing opaque film heaters and permit to see through the active layer. This

1664 could be of interest applications in health (e.g. patches for thermotherapy) or safety (e.g. control

1665 of heating elements such as automotive gas pre-heater pipes).

1666 Several relevant approaches appear very promising for $\mathrm{TH}$ production with improved

1667 functionalities. The recent development of nanomaterials has revealed unforeseen possibilities

1668 through the achievement of macroscopic performances relying on ordered or random

1669 assemblies of nanospecies, whether carbon-based or metallic. When electrically conductive

1670 nanoparticles are assembled on a surface above the percolation threshold, they give access to

1671 conductive surfaces at very low coverage values, which allows light to pass through, and to

1672 reach both high conductivity and high transparency. Another relevant possibility that needs to

1673 be further studied is the recent development based on the use of very thin films of highly

1674 conductive polymers. The performances are very promising, and these films have an

1675 intrinsically very low haze value. 


\section{WILEY-VCH}

1676 All these new technologies have managed to meet the expectations at the prototype stage, but

1677 certainly need further development to ensure reproducibility, cost-efficiency and stability at the

1678 industrial stage. Reproducibility mainly relies on the constant quality and chemical purity of

1679 the raw materials (CNTs, graphene, MNWs, polymers), but also on stable large-scale

1680 production tools and multiscale characterization techniques. Stability aspects are also

1681 particularly important, and they should be tackled while taking into account the targeted

1682 operational conditions. The stability will depend on many parameters like the structure of the

1683 device (including chemical compatibility with other layers), the applied voltage, the cyclability,

1684 high temperature long-term operating modes, and many others.

1685 Concerning the use of nanomaterials, an in-depth nanotoxicity assessment needs to be carried

1686 out to ensure the safe use for both the manufacturers' operators and the end-users. Some studies

1687 have already been reported ${ }^{[97,264-268]}$, but further understanding of all the possible toxicity 1688 aspects and long-term effects is needed.

1689 To conclude this review, the current production of THs still relies essentially on an "old" 1690 technology which has been improved upon over the years, i.e. TCOs. Many existing and future 1691 applications will keep using this technology because it is a reliable and well-proven process.

1692 However, various ongoing technical developments will provide THs with additional properties 1693 (controlled haze factor, flexibility, stretchability, low-cost deposition processes...). We expect 1694 that there will not be a single winner among them, but rather several new TH technologies that 1695 will find specific industrial applications in the near future.

1697 Acknowledgments:

1698 This project was partially supported by the French National Research Agency in the framework 1699 of the "Investissements d'avenir" program (ANR-15-IDEX-02) through the project Eco-SESA. 1700 This work was also performed within the framework of the Centre of Excellence of 


\section{WILEY-VCH}

1701 Multifunctional Architectured Materials "CEMAM" $\mathrm{n}^{\circ}$ ANR-10-LABEX-44-01 through the

1702 project Earth. This work was funded by the Agence Nationale de Recherche (ANR, France) via 1703 the program ANR-16-CE05-0021 (DESPATCH), ANR-18-CE09-0041 (Meaning) and ANR-

1704 18-CE09-0036 (Panassé). This work was also supported by the Région Auvergne Rhône-Alpes

1705 through the project Pack Ambition Recherche 2018 Eternité. The Carnot Energies du Futur is

1706 acknowledged through the project FREE. The authors would like to warmly thank Vincent 1707 Fitzpatrick for fruitful discussions.

1708

1709 


\section{WILEY-VCH}

1710

1711

1712

1713

1714

1715

1716

1717

1718

1719

1720

1721

1722

1723

1724

1725

1726

1727

1728

1729

1730

1731

1732

1733

1734

1735

1736

1737

1738

1739

1740

1741

1742

1743

1744

1745

1746

1747

1748

1749

1750

1751

1752

1753

1754

1755

1756

1757

1758

1759

\section{References:}

[1] R. Gupta, K. D. M. Rao, S. Kiruthika, G. U. Kulkarni, ACS Appl. Mater. Interfaces 2016, 8, 12559.

[2] R. G. Gordon, MRS Bulletin 2000, 25, 52.

[3] S. Long, X. Cao, G. Sun, N. Li, T. Chang, Z. Shao, P. Jin, Applied Surface Science 2018, 441, 764.

[4] L. Long, H. Ye, Scientific Reports 2015, 4, DOI 10.1038/srep06427.

[5] S. T. Heinilehto, J. H. Lappalainen, H. M. Jantunen, V. Lantto, Journal of Electroceramics 2011, 27, 7.

[6] J. You, L. Meng, T.-B. Song, T.-F. Guo, Y. (Michael) Yang, W.-H. Chang, Z. Hong, H. Chen, H. Zhou, Q. Chen, Y. Liu, N. De Marco, Y. Yang, Nature Nanotechnology 2016, 11,75 .

[7] C. G. Granquist, Thin Solid Films 2014, 564, 1.

[8] W. Gaynor, S. Hofmann, M. G. Christoforo, C. Sachse, S. Mehra, A. Salleo, M. D. McGehee, M. C. Gather, B. Lüssem, L. Müller-Meskamp, P. Peumans, K. Leo, Advanced Materials 2013, 25, 4006.

[9] K. Ellmer, Nature Photonics 2012, 6, 809.

[10] C. G. Granqvist, Solar Energy Materials and Solar Cells 2007, 91, 1529.

[11] H. J. Park, J. Kim, J. H. Won, K. S. Choi, Y. T. Lim, J. S. Shin, J.-U. Park, Thin Solid Films 2016, 615, 8.

[12] J. Gwamuri, A. Vora, J. Mayandi, D. Ö. Güney, P. L. Bergstrom, J. M. Pearce, Solar Energy Materials and Solar Cells 2016, 149, 250.

[13] M. Morales-Masis, S. De Wolf, R. Woods-Robinson, J. W. Ager, C. Ballif, Advanced Electronic Materials 2017, 3, 1600529.

[14] G. Rey, C. Ternon, M. Modreanu, X. Mescot, V. Consonni, D. Bellet, Journal of Applied Physics 2013, 114, 183713.

[15] V. Consonni, G. Rey, H. Roussel, B. Doisneau, E. Blanquet, D. Bellet, Acta Materialia 2013, 61, 22.

[16] K. Ellmer, A. Klein, B. Rech, Eds., Transparent Conductive Zinc Oxide, Springer Berlin Heidelberg, Berlin, Heidelberg, 2008.

[17] A. Klein, J. Am. Ceram. Soc. 2013, 96, 331.

[18] D. Lincot, MRS Bulletin 2010, 35, 778.

[19] V. H. Nguyen, U. Gottlieb, A. Valla, D. Muñoz, D. Bellet, D. Muñoz-Rojas, Materials Horizons 2018, 5, 715.

[20] T. Sannicolo, M. Lagrange, A. Cabos, C. Celle, J.-P. Simonato, D. Bellet, Small 2016, 12,6052 .

[21] D. S. Hecht, L. Hu, G. Irvin, Advanced Materials 2011, 23, 1482.

[22] S. Hong, H. Lee, J. Lee, J. Kwon, S. Han, Y. D. Suh, H. Cho, J. Shin, J. Yeo, S. H. Ko, Adv. Mater. 2015, 27, 4744.

[23] S. Choi, J. Park, W. Hyun, J. Kim, J. Kim, Y. B. Lee, C. Song, H. J. Hwang, J. H. Kim, T. Hyeon, D.-H. Kim, ACS Nano 2015, 9, 6626.

[24] N.-S. Jang, K.-H. Kim, S.-H. Ha, S.-H. Jung, H. M. Lee, J.-M. Kim, ACS Applied Materials \& Interfaces 2017, 9, 19612.

[25] S. Bae, H. Kim, Y. Lee, X. Xu, J.-S. Park, Y. Zheng, J. Balakrishnan, T. Lei, H. Ri Kim, Y. I. Song, Y.-J. Kim, K. S. Kim, B. Özyilmaz, J.-H. Ahn, B. H. Hong, S. Iijima, Nat Nano 2010, 5, 574.

[26] J. Zhao, Y. Li, G. Yang, K. Jiang, H. Lin, H. Ade, W. Ma, H. Yan, Nature Energy 2016, $1,15027$.

[27] Y.-H. Yoon, J.-W. Song, D. Kim, J. Kim, J.-K. Park, S.-K. Oh, C.-S. Han, Adv. Mater. 2007, 19, 4284. 


\section{WILEY-VCH}

1760 [28] J. Du, S. Pei, L. Ma, H.-M. Cheng, Advanced Materials 2014, 26, 1958.

1761 [29] Y. Sun, Y. Yin, B. T. Mayers, T. Herricks, Y. Xia, Chem. Mater. 2002, 14, 4736.

1762 [30] A. R. Rathmell, S. M. Bergin, Y.-L. Hua, Z.-Y. Li, B. J. Wiley, Advanced Materials 1763 2010, 22, 3558.

1764 [31] A. R. Rathmell, B. J. Wiley, Advanced Materials 2011, 23, 4798.

1765 [32] A. R. Rathmell, M. Nguyen, M. Chi, B. J. Wiley, Nano Letters 2012, 12, 3193.

1766 [33] S. De, T. M. Higgins, P. E. Lyons, E. M. Doherty, P. N. Nirmalraj, W. J. Blau, J. J.

1767

1768

1769

1770

1771

1772

1773

1774

1775

1776

1777

1778

1779

1780

1781

1782

1783

1784

1785

1786

1787

1788

1789

1790

1791

1792

1793

1794

1795

1796

1797

1798

1799

1800

1801

1802

1803

1804

1805

1806

1807

1808

1809

[34] S. De, P. J. King, P. E. Lyons, U. Khan, J. N. Coleman, ACS Nano 2010, 4, 7064.

[35] J. Liang, L. Li, X. Niu, Z. Yu, Q. Pei, Nature Photonics 2013, 7, 817.

[36] L. Li, Z. Yu, W. Hu, C. Chang, Q. Chen, Q. Pei, Advanced Materials 2011, 23, 5563.

[37] S. Ye, A. R. Rathmell, Z. Chen, I. E. Stewart, B. J. Wiley, Adv. Mater. 2014, 26, 6670.

[38] D. Bellet, M. Lagrange, T. Sannicolo, S. Aghazadehchors, V. H. Nguyen, D. P.

Langley, D. Muñoz-Rojas, C. Jiménez, Y. Bréchet, N. D. Nguyen, Materials 2017, 10, 570.

[39] D. Chen, J. Liang, Q. Pei, Sci. China Chem. 2016, 1.

[40] J. Liu, D. Jia, J. M. Gardner, E. M. J. Johansson, X. Zhang, Materials Today Energy 2019, 13, 152.

[41] R. Zhang, M. Engholm, R. Zhang, M. Engholm, Nanomaterials 2018, 8, 628.

[42] C. Celle, C. Mayousse, E. Moreau, H. Basti, A. Carella, J.-P. Simonato, Nano Research 2012, 5, 427.

[43] S. Sorel, D. Bellet, J. N. Coleman, ACS Nano 2014, 8, 4805.

[44] O. Ergun, S. Coskun, Y. Yusufoglu, H. E. Unalan, Nanotechnology 2016, 27, 445708.

[45] T. Kim, Y. W. Kim, H. S. Lee, H. Kim, W. S. Yang, K. S. Suh, Advanced Functional Materials 2013, 23, 1250.

[46] M. Lagrange, T. Sannicolo, D. Muñoz-Rojas, B. G. Lohan, A. Khan, M. Anikin, C. Jiménez, F. Bruckert, Y. Bréchet, D Bellet, Nanotechnology 2017, 28, 055709.

[47] A. Khan, V. H. Nguyen, D. Muñoz-Rojas, S. Aghazadehchors, C. Jiménez, N. D. Nguyen, D. Bellet, ACS Applied Materials \& Interfaces 2018, 10, 19208.

[48] C. Celle, A. Cabos, T. Fontecave, B. Laguitton, A. Benayad, L. Guettaz, N. Pélissier, V. H. Nguyen, D. Bellet, D. Muñoz-Rojas, J.-P. Simonato, Nanotechnology 2018, 29, 085701.

[49] M. N. Gueye, A. Carella, N. Massonnet, E. Yvenou, S. Brenet, J. Faure-Vincent, S. Pouget, F. Rieutord, H. Okuno, A. Benayad, R. Demadrille, J.-P. Simonato, Chemistry of Materials 2016, 28, 3462.

[50] M. N. Gueye, A. Carella, R. Demadrille, J.-P. Simonato, ACS Appl. Mater. Interfaces 2017, 9, 27250.

[51] E.-H. Ko, H.-J. Kim, S.-J. Lee, J.-H. Lee, H.-K. Kim, RSC Advances 2016, 6, 46634.

[52] S.-H. Park, S.-M. Lee, E.-H. Ko, T.-H. Kim, Y.-C. Nah, S.-J. Lee, J. H. Lee, H.-K. Kim, Scientific Reports 2016, 6, DOI 10.1038/srep33868.

[53] H. Kim, Y. Kim, K. S. Kim, H. Y. Jeong, A.-R. Jang, S. H. Han, D. H. Yoon, K. S. Suh, H. S. Shin, T. Kim, W. S. Yang, ACS Nano 2013, 7, 5769.

[54] H. H. Khaligh, L. Xu, A. Khosropour, A. Madeira, M. Romano, C. Pradére, M. Tréguer-Delapierre, L. Servant, M. A. Pope, I. A. Goldthorpe, Nanotechnology 2017, 28, 425703.

[55] G. Haacke, Journal of Applied Physics 1976, 47, 4086.

[56] X. Chen, S. Nie, W. Guo, F. Fei, W. Su, W. Gu, Z. Cui, Advanced Electronic Materials 2019, 5, 1800991.

[57] J. Kang, H. Kim, K. S. Kim, S.-K. Lee, S. Bae, J.-H. Ahn, Y.-J. Kim, J.-B. Choi, B. H. Hong, Nano Lett. 2011, 11, 5154. 


\section{WILEY-VCH}

1810

1811

1812

1813

1814

1815

1816

1817

1818

1819

1820

1821

1822

1823

1824

1825

1826

1827

1828

1829

1830

1831

1832

1833

1834

1835

1836

1837

1838

1839

1840

1841

1842

1843

1844

1845

1846

1847

1848

1849

1850

1851

1852

1853

1854

1855

1856

1857

1858

1859

1860

[58] H. Askari, H. Fallah, M. Askari, M. C. Mohmmadieyh, arXiv:1409.5293 [cond-mat] 2014.

[59] G. Giusti, V. Consonni, E. Puyoo, D. Bellet, ACS Appl. Mater. Interfaces 2014, 6, 14096.

[60] S. Ke, J. Xie, C. Chen, P. Lin, X. Zeng, L. Shu, L. Fei, Y. Wang, M. Ye, D. Wang, Applied Physics Letters 2018, 112, 031905.

[61] Y. Kim, H. R. Lee, T. Saito, Y. Nishi, Applied Physics Letters 2017, 110, 153301.

[62] H. Choi, J. S. Choi, J.-S. Kim, J.-H. Choe, K. H. Chung, J.-W. Shin, J. T. Kim, D.-H. Youn, K.-C. Kim, J.-I. Lee, S.-Y. Choi, P. Kim, C.-G. Choi, Y.-J. Yu, Small 2014, 10, 3685.

[63] P. Blake, P. D. Brimicombe, R. R. Nair, T. J. Booth, D. Jiang, F. Schedin, L. A. Ponomarenko, S. V. Morozov, H. F. Gleeson, E. W. Hill, A. K. Geim, K. S. Novoselov, Nano Lett. 2008, 8, 1704.

[64] M. Lagrange, D. P. Langley, G. Giusti, C. Jiménez, Y. Bréchet, D. Bellet, Nanoscale 2015, 7, 17410.

[65] H. Yang, S. Bai, X. Guo, H. Wang, Applied Surface Science 2019, 483, 888.

[66] M. Bobinger, J. Mock, P. La Torraca, M. Becherer, P. Lugli, L. Larcher, Advanced Materials Interfaces 2017, 4, 1700568.

[67] K. D. M. Rao, G. U. Kulkarni, Nanoscale 2014, 6, 5645.

[68] H. Hu, S. Wang, S. Wang, G. Liu, T. Cao, Y. Long, Advanced Functional Materials 2019, 1902922.

[69] H. Wu, D. Kong, Z. Ruan, P.-C. Hsu, S. Wang, Z. Yu, T. J. Carney, L. Hu, S. Fan, Y. Cui, Nat Nano 2013, 8, 421.

[70] B. W. An, E.-J. Gwak, K. Kim, Y.-C. Kim, J. Jang, J.-Y. Kim, J.-U. Park, Nano Letters 2016, $16,471$.

[71] R. Gupta, K. D. M. Rao, K. Srivastava, A. Kumar, S. Kiruthika, G. U. Kulkarni, ACS Applied Materials \& Interfaces 2014, 6, 13688.

[72] D. Lordan, M. Burke, M. Manning, A. Martin, A. Amann, D. O’Connell, R. Murphy, C. Lyons, A. J. Quinn, ACS Applied Materials \& Interfaces 2017, 9, 4932.

[73] H. Wang, S. Lin, D. Zu, J. Song, Z. Liu, L. Li, C. Jia, X. Bai, J. Liu, Z. Li, D. Wang, Y. Huang, M. Fang, M. Lei, B. Li, H. Wu, Advanced Materials Technologies 2019, 1900045.

[74] A. Khan, S. Lee, T. Jang, Z. Xiong, C. Zhang, J. Tang, L. J. Guo, W.-D. Li, Small 2016, 12, 3021.

[75] S. An, H. S. Jo, D.-Y. Kim, H. J. Lee, B.-K. Ju, S. S. Al-Deyab, J.-H. Ahn, Y. Qin, M. T. Swihart, A. L. Yarin, S. S. Yoon, Advanced Materials 2016, 28, 7149.

[76] H. S. Jo, S. An, J.-G. Lee, H. G. Park, S. S. Al-Deyab, A. L. Yarin, S. S. Yoon, NPG Asia Materials 2017, 9, e347.

[77] N. Kim, H. Kang, J.-H. Lee, S. Kee, S. H. Lee, K. Lee, Advanced Materials 2015, 27, 2317.

[78] Y. Wang, C. Zhu, R. Pfattner, H. Yan, L. Jin, S. Chen, F. Molina-Lopez, F. Lissel, J. Liu, N. I. Rabiah, Z. Chen, J. W. Chung, C. Linder, M. F. Toney, B. Murmann, Z. Bao, Science Advances 2017, 3, e1602076.

[79] J. Li, J. Liang, X. Jian, W. Hu, J. Li, Q. Pei, Macromol. Mater. Eng. 2014, 299, 1403.

[80] B.-Y. Hwang, S.-H. Choi, K.-W. Lee, J.-Y. Kim, Composites Part B: Engineering 2018, 151, 1 .

[81] S. B. Singh, Y. Hu, T. Kshetri, N. H. Kim, J. H. Lee, Journal of Materials Chemistry C 2017, 5, 4198.

[82] S. Kiruthika, R. Gupta, G. U. Kulkarni, RSC Adv. 2014, 4, 49745.

[83] J. J. Bae, S. C. Lim, G. H. Han, Y. W. Jo, D. L. Doung, E. S. Kim, S. J. Chae, T. Q. Huy, N. Van Luan, Y. H. Lee, Adv. Funct. Mater. 2012, 22, 4819. 


\section{WILEY-VCH}

1861 [84] A.-Y. Kim, K. Lee, J. H. Park, D. Byun, J. K. Lee, physica status solidi (a) 2014, 211, 1923.

1863

1864

1865

1866

1867

1868

1869

1870

1871

1872

1873

1874

1875

1876

1877

1878

1879

1880

1881

1882

1883

1884

1885

1886

1887

1888

1889

1890

1891

1892

1893

1894

1895

1896

1897

1898

1899

1900

1901

1902

1903

1904

1905

1906

1907

1908

1909

1910

[85] S. Duzyer, S. Sinha-Ray, S. Sinha-Ray, A. L. Yarin, Macromolecular Materials and Engineering 2017, 302, 1700188.

[86] D. Sui, Y. Huang, L. Huang, J. Liang, Y. Ma, Y. Chen, Small 2011, 7, 3186.

[87] S. Ji, J. Park, Y. Jo, Y.-B. Kim, J. Jang, S.-K. Kim, S. Jeong, J.-U. Park, Applied Surface Science 2019, 483, 1101.

[88] T. J. Kang, T. Kim, S. M. Seo, Y. J. Park, Y. H. Kim, Carbon 2011, 49, 1087.

[89] S. R. Das, A. M. S. Mohammed, K. Maize, S. Sadeque, A. Shakouri, D. B. Janes, M. A. Alam, Nano Lett. 2016, 16, 3130.

[90] D. P. Langley, M. Lagrange, G. Giusti, C. Jiménez, Y. Bréchet, N. D. Nguyen, D. Bellet, Nanoscale 2014, 6, 13535.

[91] K. Maize, S. R. Das, S. Sadeque, A. M. S. Mohammed, A. Shakouri, D. B. Janes, M. A. Alam, Applied Physics Letters 2015, 106, 143104.

[92] F. M. Smits, Bell System Technical Journal 1958, 37, 711.

[93] T. Sannicolo, N. Charvin, L. Flandin, S. Kraus, D. T. Papanastasiou, C. Celle, J.-P. Simonato, D. Muñoz-Rojas, C. Jiménez, D. Bellet, ACS Nano 2018, 12, 4648.

[94] N. Hoof, M. Parente, A. Baldi, J. G. Rivas, Advanced Optical Materials 2019, 1900790.

[95] S.-T. Zhang, M. Foldyna, H. Roussel, V. Consonni, E. Pernot, L. Schmidt-Mende, L. Rapenne, C. Jiménez, J.-L. Deschanvres, D. Muñoz-Rojas, D. Bellet, J. Mater. Chem. $C$ 2016, $5,91$.

[96] T. Araki, J. Jiu, M. Nogi, H. Koga, S. Nagao, T. Sugahara, K. Suganuma, Nano Res. 2014, 7, 236.

[97] D. Toybou, C. Celle, C. Aude-Garcia, T. Rabilloud, J.-P. Simonato, Environmental Science: Nano 2019, 6, 684.

[98] P. R. N. Childs, in Practical Temperature Measurement, Elsevier, 2001, pp. 98-144.

[99] B. Liptak, Ed., Instrument Engineers' Handbook, Fourth Edition, Volume One: Process Measurement and Analysis, CRC Press, 2003.

[100] A. Rogalski, P. Martyniuk, M. Kopytko, Reports on Progress in Physics 2016, 79, 046501.

[101] Y.-A. Li, Y.-J. Chen, N.-H. Tai, Materials Research Express 2014, 1, 025605.

[102] D. Kim, L. Zhu, D.-J. Jeong, K. Chun, Y.-Y. Bang, S.-R. Kim, J.-H. Kim, S.-K. Oh, Carbon 2013, 63, 530.

[103] D. Pierścińska, Journal of Physics D: Applied Physics 2018, 51, 013001.

[104] T. Yagi, K. Tamano, Y. Sato, N. Taketoshi, T. Baba, Y. Shigesato, Journal of Vacuum Science \& Technology A: Vacuum, Surfaces, and Films 2005, 23, 1180.

[105] O. Breitenstein, W. Warta, M. Langenkamp, Lock-in Thermography: Basics and Use for Evaluating Electronic Devices and Materials, Springer Science \& Business Media, 2010.

[106] Ch. Schmidt, F. Altmann, O. Breitenstein, Materials Science and Engineering: B 2012, $177,1261$.

[107] T. Sannicolo, D. Muñoz-Rojas, N. D. Nguyen, S. Moreau, C. Celle, J.-P. Simonato, Y. Bréchet, D. Bellet, Nano Letters 2016, DOI 10.1021/acs.nanolett.6b03270.

[108] R. Gupta, A. Kumar, S. Sadasivam, S. Walia, G. U. Kulkarni, T. S. Fisher, A. Marconnet, ACS Applied Materials \& Interfaces 2017, 9, 13703.

[109] H. Kim, H. Lee, I. Ha, J. Jung, P. Won, H. Cho, J. Yeo, S. Hong, S. Han, J. Kwon, K.J. Cho, S. H. Ko, Advanced Functional Materials 2018, 28, 1801847.

[110] V. Zardetto, T. M. Brown, A. Reale, A. Di Carlo, Journal of Polymer Science Part B: Polymer Physics 2011, 49, 638. 


\section{WILEY-VCH}

1911 [111] J. Jang, B. G. Hyun, S. Ji, E. Cho, B. W. An, W. H. Cheong, J.-U. Park, NPG Asia

1912

1913

1914

1915

1916

1917

1918

1919

1920

1921

1922

1923

1924

1925

1926

1927

1928

1929

1930

1931

1932

1933

1934

1935

1936

1937

1938

1939

1940

1941

1942

1943

1944

1945

1946

1947

1948

1949

1950

1951

1952

1953

1954

1955

1956

1957

1958

1959

1960 Materials 2017, 9, e432.

[112] S. M. Lee, S. Oh, S. T. Chang, ACS Applied Materials \& Interfaces 2019, 11, 4541.

[113] F. Oytun, O. Alpturk, F. Basarir, Materials Research Bulletin 2019, 112, 53.

[114] J.-G. Lee, J.-H. Lee, S. An, D.-Y. Kim, T.-G. Kim, S. S. Al-Deyab, A. L. Yarin, S. S. Yoon, Journal of Materials Chemistry A 2017, 5, 6677.

[115] H.-J. Kim, Y. Kim, J.-H. Jeong, J.-H. Choi, J. Lee, D.-G. Choi, Journal of Materials Chemistry A 2015, 3, 16621.

[116] M. Cao, M. Wang, L. Li, H. Qiu, Z. Yang, ACS Applied Materials \& Interfaces 2018, 10, 1077.

[117] D. Doganay, S. Coskun, S. P. Genlik, H. E. Unalan, Nanotechnology 2016, 27, 435201.

[118] S. Xie, T. Li, Z. Xu, Y. Wang, X. Liu, W. Guo, Nanoscale 2018, 10, 6531.

[119] Y. Tang, H. Ruan, Z. Huang, D. Shi, H. Liu, S. Chen, J. Zhang, Nanotechnology 2018, 29, 455706.

[120] J. Kwon, H. Cho, Y. D. Suh, J. Lee, H. Lee, J. Jung, D. Kim, D. Lee, S. Hong, S. H. Ko, Advanced Materials Technologies 2017, 2, 1600222.

[121] W. Lan, Y. Chen, Z. Yang, W. Han, J. Zhou, Y. Zhang, J. Wang, G. Tang, Y. Wei, W. Dou, Q. Su, E. Xie, ACS Applied Materials \& Interfaces 2017, 9, 6644.

[122] E. C. David Levy, Ed., TRANSPARENT CONDUCTIVE MATERIALS: From Materials via Synthesis and Characterization to Applications., WILEY-VCH, Place of Publication Not Identified, 2019.

[123] D. Ginley, H. Hosono, D. C. Paine, Handbook of Transparent Conductors, Springer Science \& Business Media, 2010.

[124] V. Consonni, G. Rey, H. Roussel, D. Bellet, Journal of Applied Physics 2012, 111, 033523.

[125] I. Hamberg, C. G. Granqvist, Journal of Applied Physics 1986, 60, R123.

[126] B. D. Ahn, S. H. Oh, D. U. Hong, D. H. Shin, A. Moujoud, H. J. Kim, Journal of Crystal Growth 2008, 310, 3303.

[127] D. S. Y. Jayathilake, J. S. Sagu, K. G. U. Wijayantha, Materials Letters 2018, DOI 10.1016/j.matlet.2018.11.092.

[128] J. H. Kim, B. D. Ahn, C. H. Kim, K. A. Jeon, H. S. Kang, S. Y. Lee, Thin Solid Films 2008, 516, 1330.

[129] M. K. Roul, B. Obasogie, G. Kogo, J. R. Skuza, R. M. Mundle, A. K. Pradhan, Journal of Applied Physics 2017, 122, 135110.

[130] K. Im, K. Cho, J. Kim, S. Kim, Thin Solid Films 2010, 518, 3960.

[131] C. Kim, J.-W. Park, J. Kim, S.-J. Hong, M. J. Lee, Journal of Alloys and Compounds 2017, 726, 712.

[132] K. Yang, K. Cho, K. Im, S. Kim, Materials Research Bulletin 2015, 63, 194.

[133] S. Ke, C. Chen, N. Fu, H. Zhou, M. Ye, P. Lin, W. Yuan, X. Zeng, L. Chen, H. Huang, ACS Applied Materials \& Interfaces 2016, 8, 28406.

[134] V. H. Nguyen, D. Bellet, B. Masenelli, D. Muñoz-Rojas, ACS Applied Nano Materials 2018, $1,6922$.

[135] L. Li, S. K. Hong, Y. Jo, M. Tian, C. Y. Woo, S. H. Kim, J.-M. Kim, H. W. Lee, ACS Applied Materials \& Interfaces 2019, 11, 16223.

[136] X. Yao, S. C. Hawkins, B. G. Falzon, Carbon 2018, 136, 130.

[137] N. Karim, M. Zhang, S. Afroj, V. Koncherry, P. Potluri, K. S. Novoselov, RSC Advances 2018, 8, 16815.

[138] H. Souri, D. Bhattacharyya, ACS Applied Materials \& Interfaces 2018, 10, 20845.

[139] M. Yang, J. Pan, A. Xu, L. Luo, D. Cheng, G. Cai, J. Wang, B. Tang, X. Wang, Polymers 2018, 10, 568. 


\section{WILEY-VCH}

1961 [140] P. Liu, D. Zhou, Y. Wei, K. Jiang, J. Wang, L. Zhang, Q. Li, S. Fan, ACS Nano 2015,

1962

1963

1964

1965

1966

1967

1968

1969

1970

1971

1972

1973

1974

1975

1976

1977

1978

1979

1980

1981

1982

1983

1984

1985

1986

1987

1988

1989

1990

1991

1992

1993

1994

1995

1996

1997

1998

1999

2000

2001

2002

2003

2004

2005

2006

2007

2008

2009

2010

2011 9, 3753.

[141] B. Zhou, X. Han, L. Li, Y. Feng, T. Fang, G. Zheng, B. Wang, K. Dai, C. Liu, C. Shen, Composites Science and Technology 2019, 183, 107796.

[142] H. Souri, S. J. Yu, H. Yeo, M. Goh, J.-Y. Hwang, S. M. Kim, B.-C. Ku, Y. G. Jeong, N.-H. You, RSC Advances 2016, 6, 52509.

[143] O. P. Morris, X. Zang, A. Gregg, B. Keller, B. Getachew, S. Ingersoll, H. A. Elsen, M. M. Disko, N. Ferralis, J. C. Grossman, Advanced Materials 2019, 31, 1900331.

[144] E. J. Spadafora, K. Saint-Aubin, C. Celle, R. Demadrille, B. Grévin, J.-P. Simonato, Carbon 2012, 50, 3459.

[145] H.-S. Jang, S. K. Jeon, S. H. Nahm, Carbon 2011, 49, 111.

[146] T. L. Chen, D. S. Ghosh, M. Marchena, J. Osmond, V. Pruneri, ACS Applied Materials \& Interfaces 2015, 7, 5938.

[147] L. Hu, H. Wu, Y. Cui, MRS Bulletin 2011, 36, 760.

[148] B.-J. Kim, J.-S. Park, R. Yoo, J.-S. Park, RSC Advances 2017, 7, 53025.

[149] N. Kwon, K. Kim, J. Heo, I. Yi, I. Chung, Nanotechnology 2014, 25, 265702.

[150] P. Li, J. G. Ma, H. Y. Xu, D. Lin, X. D. Xue, X. Z. Yan, P. Xia, Y. C. Liu, Journal of Alloys and Compounds 2016, 664, 764.

[151] A. Khan, C. Liang, Y.-T. Huang, C. Zhang, J. Cai, S.-P. Feng, W.-D. Li, Advanced Engineering Materials 2019, 1900723.

[152] E. Thouti, C. Mistry, A. Chandran, D. K. Panwar, P. Kumar, H. Suman, J. Akhtar, Journal of Physics D: Applied Physics 2019, DOI 10.1088/1361-6463/ab31dc.

[153] T. Sannicolo, Transparent Electrodes Based on Silver Nanowire Networks: Electrical Percolation, Physical Properties, and Applications, PhD thesis, University of Grenoble Alpes (France), 2017.

[154] J. H. Park, D. Y. Lee, Y.-H. Kim, J. K. Kim, J. H. Lee, J. H. Park, T.-W. Lee, J. H. Cho, ACS Appl. Mater. Interfaces 2014, 6, 12380.

[155] Y.-Y. Zhao, M.-L. Zheng, X.-Z. Dong, F. Jin, J. Liu, X.-L. Ren, X.-M. Duan, Z.-S. Zhao, Applied Physics Letters 2016, 108, 221104.

[156] J. Kang, C.-G. Park, S.-H. Lee, C. Cho, D.-G. Choi, J.-Y. Lee, Nanoscale 2016, 8, 11217.

[157] J. Xue, J. Song, Y. Dong, L. Xu, J. Li, H. Zeng, Science Bulletin 2017, 62, 143.

[158] H. Sohn, C. Park, J.-M. Oh, S. W. Kang, M.-J. Kim, Materials 2019, 12, 2526.

[159] C. G. da Rocha, H. G. Manning, C. O'Callaghan, C. Ritter, A. T. Bellew, J. J. Boland, M. S. Ferreira, Nanoscale 2015, 7, 13011.

[160] A. T. Bellew, H. G. Manning, C. Gomes da Rocha, M. S. Ferreira, J. J. Boland, ACS Nano 2015, 9, 11422.

[161] S. Coskun, E. Selen Ates, H. Emrah Unalan, Nanotechnology 2013, 24, 125202.

[162] Y. Goliya, A. Rivadeneyra, J. F. Salmeron, A. Albrecht, J. Mock, M. Haider, J. Russer, B. Cruz, P. Eschlwech, E. Biebl, M. Becherer, M. R. Bobinger, Advanced Optical Materials 2019, 1900995.

[163] M. Bobinger, J. Mock, M. Becherer, P. L. Torraca, D. Angeli, L. Larcher, P. Lugli, IEEE, 2017, pp. 151-154.

[164] I. E. Stewart, S. Ye, Z. Chen, P. F. Flowers, B. J. Wiley, Chemistry of Materials 2015, $27,7788$.

[165] E. C. Garnett, W. Cai, J. J. Cha, F. Mahmood, S. T. Connor, M. Greyson Christoforo, Y. Cui, M. D. McGehee, M. L. Brongersma, Nature Materials 2012, 11, 241.

[166] Y. Huang, Y. Tian, C. Hang, Y. Liu, S. Wang, M. Qi, H. Zhang, J. Zhao, ACS Applied Materials \& Interfaces 2019, 11, 21850.

[167] D. Chen, F. Zhao, K. Tong, G. Saldanha, C. Liu, Q. Pei, Advanced Electronic Materials 2016, 2, 1600167. 


\section{WILEY-VCH}

2012 [168] H. H. Khaligh, I. A. Goldthorpe, Nanoscale research letters 2013, 8, 1.

2013

[169] D. Tigan, S. P. Genlik, B. Imer, H. E. Unalan, Nanotechnology 2019, 30, 325202.

2014

2015

2016

2017

2018

2019

2020

2021

2022

2023

2024

2025

2026

2027

2028

2029

2030

2031

2032

2033

2034

2035

2036

2037

2038

2039

2040

2041

2042

2043

2044

2045

2046

2047

2048

2049

2050

2051

2052

2053

2054

2055

2056

2057

2058

2059

2060

[170] M.-G. Kang, L. J. Guo, Adv. Mater. 2007, 19, 1391.

[171] B. Bessaire, M. Mathieu, V. Salles, T. Yeghoyan, C. Celle, J.-P. Simonato, A. Brioude, ACS Applied Materials \& Interfaces 2017, 9, 950.

[172] L. J. Romasanta, P. Schäfer, J. Leng, Scientific Reports 2018, 8, DOI 10.1038/s41598018-34538-w.

[173] M. N. Gueye, A. Carella, J. Faure-Vincent, R. Demadrille, J.-P. Simonato, Progress in Materials Science 2019, 100616.

[174] C. Shi, K. A. Owusu, X. Xu, T. Zhu, G. Zhang, W. Yang, L. Mai, Small 2019, 1902348.

[175] X. He, R. He, Q. Lan, W. Wu, F. Duan, J. Xiao, M. Zhang, Q. Zeng, J. Wu, J. Liu, Materials 2017, 10, 220.

[176] X. He, G. Shen, R. Xu, W. Yang, C. Zhang, Z. Liu, B. Chen, J. Liu, M. Song, Polymers 2019, 11, 468.

[177] J. Yan, Y. G. Jeong, Materials \& Design 2015, 86, 72.

[178] S.-B. Yang, H. Choi, D. S. Lee, C.-G. Choi, S.-Y. Choi, I.-D. Kim, Small 2015, 11, 1293.

[179] L. Dou, F. Cui, Y. Yu, G. Khanarian, S. W. Eaton, Q. Yang, J. Resasco, C. Schildknecht, K. Schierle-Arndt, P. Yang, ACS Nano 2016, 10, 2600.

[180] H. J. Han, Y. C. Choi, J. H. Han, Synthetic Metals 2015, 199, 219.

[181] Y. Ahn, Y. Jeong, D. Lee, Y. Lee, ACS Nano 2015, 9, 3125.

[182] C. Wu, J. Jiu, T. Araki, H. Koga, T. Sekitani, H. Wang, K. Suganuma, RSC Adv. 2016, 6,15838 .

[183] Y. Tang, H. Ruan, Y. Chen, J. Xiang, H. Liu, R. Jin, D. Shi, S. Chen, J. Zhang, Nanotechnology 2020, 31, 045704.

[184] S. M. Lee, J. H. Lee, S. Bak, K. Lee, Y. Li, H. Lee, Nano Research 2015, 8, 1882.

[185] Y. Cai, X. Piao, X. Yao, W. Gao, E. Nie, Z. Zhang, Z. Sun, Nanotechnology 2019, 30, 225201.

[186] M. P. Gupta, N. Kumar, S. Kumar, IEEE Transactions on Nanotechnology 2018, 17, 829.

[187] S. Sadeque, Y. Gong, K. Maize, A. K. Ziabari, A. M. Mohammed, A. Shakouri, D. B. Janes, IEEE Transactions on Nanotechnology 2018, 17, 276.

[188] S. Wang, Y. Tian, C. Wang, C. Hang, Y. Huang, C. Liao, Composites Science and Technology 2019, 174, 76.

[189] K. Pyo, J.-W. Kim, Current Applied Physics 2016, 16, 1453.

[190] D. Han, Y. Li, X. Jiang, W. Zhao, F. Wang, W. Lan, E. Xie, W. Han, Composites Science and Technology 2018, 168, 460.

[191] K. Barros, P. L. Krapivsky, S. Redner, Physical Review E 2009, 80, DOI 10.1103/PhysRevE.80.040101.

[192] M. Žeželj, I. Stanković, A. Belić, Physical Review E 2012, 85, DOI 10.1103/PhysRevE.85.021101.

[193] J.-S. Bae, Y.-S. Lee, J. Li, J. Liang, D. Chen, Q. Pei, S.-B. Lee, Advanced Materials Technologies 2018, 1700364.

[194] S. Ji, W. He, K. Wang, Y. Ran, C. Ye, Small 2014, 10, 4951.

[195] K. Lee, J. Park, H. Kim, H.-S. Park, H.-K. Song, K.-H. Kim, K. Seo, Journal of Materials Chemistry A 2018.

[196] C.-Y. Chou, H.-S. Liu, G.-S. Liou, RSC Advances 2016, 6, 61386.

[197] M. Patel, K. R. Chauhan, J. Kim, J.-W. Kim, D. Lim, Sensors and Actuators A: Physical 2017, 267, 8.

[198] Y. Cai, X. Piao, X. Yao, E. Nie, Z. Zhang, Z. Sun, Materials Letters 2019, 249, 66. 


\section{WILEY-VCH}

2063

2064

2065

2066

2067

2068

2069

2070

2071

2072

2073

2074

2075

2076

2077

2078

2079

2080

2081

2082

2083

2084

2085

2086

2087

2088

2089

2090

2091

2092

2093

2094

2095

2096

2097

2098

2099

2100

2101

2102

2103

2104

2105

2106

2107

2108

2109

2110

2111

2112
[199] X. Shi, W. Xu, W. Shen, G. Wang, R. Wang, X. Li, W. Song, Journal of Materials Science: Materials in Electronics 2018, DOI 10.1007/s10854-018-0480-4.

[200] W.-R. Huang, Z. He, J.-L. Wang, J.-W. Liu, S.-H. Yu, iScience 2019, 12, 333.

[201] P. Li, J. Ma, H. Xu, X. Xue, Y. Liu, J. Mater. Chem. C 2016, 4, 3581.

[202] N. Tiwari, A. Ankit, M. Rajput, M. R. Kulkarni, R. A. John, N. Mathews, Nanoscale 2017, 9, 14990.

[203] X. Shi, W. Xu, J. Zhang, R. Wang, X. Li, W. Shen, G. Chen, X. Fang, W. Song, Materials Research Express 2019, 6, 095069.

[204] H.-Y. Lu, C.-Y. Chou, J.-H. Wu, J.-J. Lin, G.-S. Liou, Journal of Materials Chemistry C 2015, 3, 3629.

[205] Z. R. Ramadhan, J. W. Han, D. J. Lee, S. A. N. Entifar, J. Hong, C. Yun, Y. H. Kim, Materials Research Letters 2019, 7, 124.

[206] J. Li, S. Qi, J. Liang, L. Li, Y. Xiong, W. Hu, Q. Pei, ACS Applied Materials \& Interfaces 2015, 7, 14140.

[207] F. S. F. Morgenstern, D. Kabra, S. Massip, T. J. K. Brenner, P. E. Lyons, J. N. Coleman, R. H. Friend, Applied Physics Letters 2011, 4, 183307.

[208] V. H. Nguyen, J. Resende, D. T. Papanastasiou, N. Fontanals, C. Jiménez, D. MuñozRojas, D. Bellet, Nanoscale 2019, 11, 12097.

[209] D. Muñoz-Rojas, V. H. Nguyen, C. Masse de la Huerta, S. Aghazadehchors, C. Jiménez, D. Bellet, Comptes Rendus Physique 2017, 18, 391.

[210] V. H. Nguyen, J. Resende, C. Jiménez, J.-L. Deschanvres, P. Carroy, D. Muñoz, D. Bellet, D. Muñoz-Rojas, Journal of Renewable and Sustainable Energy 2017, 9, 021203.

[211] S. Aghazadehchors, V. H. Nguyen, D. Muñoz-Rojas, C. Jiménez, L. Rapenne, N. D. Nguyen, D. Bellet, Nanoscale 2019, 11, 19969.

[212] H.-G. Cheong, J.-H. Kim, J.-H. Song, U. Jeong, J.-W. Park, Thin Solid Films 2015, $589,633$.

[213] H. Chen, L. Wang, J. Li, Y. Yu, X. Bi, Materials Letters 2018, $217,52$.

[214] B. Sharma, J.-S. Kim, A. Sharma, Microelectronic Engineering 2019, 205, 37.

[215] J. Kang, Y. Jang, Y. Kim, S.-H. Cho, J. Suhr, B. H. Hong, J.-B. Choi, D. Byun, Nanoscale 2015, 7, 6567.

[216] H. Khachatryan, M. Kim, H.-J. Seok, H.-K. Kim, Materials Science in Semiconductor Processing 2019, 99, 1.

[217] D.-H. Kim, K.-S. Cho, H.-K. Kim, Scientific Reports 2017, 7, DOI 10.1038/s41598017-02711-2.

[218] M. K. Roul, S. K. Pradhan, K. D. Song, M. J. Bahoura, Journal of Materials Science 2019, 54, 7062 .

[219] T.-W. Kang, S. H. Kim, C. H. Kim, S.-M. Lee, H.-K. Kim, J. S. Park, J. H. Lee, Y. S. Yang, S.-J. Lee, ACS Applied Materials \& Interfaces 2017, 9, 33129.

[220] G. Kim, J. W. Lim, C. Yeon, T. Kim, H. C. Lee, S. J. Yun, Journal of Alloys and Compounds 2019, 774, 1092.

[221] H. Im, E. Y. Jang, A. Choi, W. J. Kim, T. J. Kang, Y. W. Park, Y. H. Kim, ACS Applied Materials \& Interfaces 2012, 4, 2338.

[222] C. Hudaya, B. J. Jeon, J. K. Lee, ACS Appl. Mater. Interfaces 2015, 7, 57.

[223] K.-D. Kim, T. Pfadler, E. Zimmermann, Y. Feng, J. A. Dorman, J. Weickert, L. Schmidt-Mende, APL Materials 2015, 3, 106105.

[224] Y. Cheng, H. Zhang, R. Wang, X. Wang, H. Zhai, T. Wang, Q. Jin, J. Sun, ACS Applied Materials \& Interfaces 2016, 8, 32925.

[225] R. Singh, R. Kuzhikkali, N. Shet, N. Sekarapandian, G. Kizhedath, M. Arumugum, SABIC Tech Center, 2016, p. 9. 


\section{WILEY-VCH}

2113

2114

2115

2116

2117

2118

2119

2120

2121

2122

2123

2124

2125

2126

2127

2128

2129

2130

2131

2132

2133

2134

2135

2136

2137

2138

2139

2140

2141

2142

2143

2144

2145

2146

2147

2148

2149

2150

2151

2152

2153

2154

2155

2156

2157

2158

2159

2160

2161

2162

2163

[226] B. Vandecastele, Heated window for refrigerated showcase and method of manufacturing same, 1992, EP0502775.

[227] "Heater Circuits and LCD monitors," can be found under https://www.allflexinc.com/blog/heater-circuits-and-lcd-monitors/, 2015.

[228] "Transparent Heaters and LCD Heaters," can be found under http://touchinternational.com/products/touch-display-enhancements/transparentheaters/, n.d.

[229] S.-S. Yoon, D.-Y. Khang, ACS Applied Materials \& Interfaces 2016, 8, 23236.

[230] L. Veeramuthu, B.-Y. Chen, C.-Y. Tsai, F.-C. Liang, M. Venkatesan, D.-H. Jiang, C.W. Chen, X. Cai, C.-C. Kuo, RSC Advances 2019, 9, 35786.

[231] S.-W. Kim, B. W. An, E. Cho, B. G. Hyun, Y.-J. Moon, S.-K. Kim, J.-U. Park, Nano Letters 2018, 18, 3865.

[232] W. Cheong, Y. Kim, J. Lee, C. Hong, H. Choi, Y. Kwak, Y. J. Kim, Y. S. Kim, Advanced Materials Technologies 2019, 4, 1800550.

[233] Y. Cheng, X. Zhang, C. Fang, J. Chen, Z. Wang, Journal of Materials Science \& Technology 2018, 34, 2225.

[234] Y. Gao, H. Luo, Z. Zhang, L. Kang, Z. Chen, J. Du, M. Kanehira, C. Cao, Nano Energy 2012, 1, 221.

[235] Z. Shao, X. Cao, H. Luo, P. Jin, NPG Asia Materials 2018, 10, 581.

[236] P. Liu, L. Liu, K. Jiang, S. Fan, Small 2011, 7, 732.

[237] M. Li, S. Ji, J. Pan, H. Wu, L. Zhong, Q. Wang, F. Li, G. Li, J. Mater. Chem. A 2014, 2, 20470.

[238] Q. Huang, W. Shen, X. Fang, G. Chen, J. Guo, W. Xu, R. Tan, W. Song, RSC Advances 2015, 5, 45836.

[239] S. Yao, J. Cui, Z. Cui, Y. Zhu, Nanoscale 2017, 9, 3797.

[240] Xing Liang, S. A. Boppart, IEEE Transactions on Biomedical Engineering 2010, 57, 953.

[241] Y. Liu, M. Pharr, G. A. Salvatore, ACS Nano 2017, 11, 9614.

[242] M. Amjadi, K.-U. Kyung, I. Park, M. Sitti, Advanced Functional Materials 2016, 26, 1678.

[243] S. Bagherifard, A. Tamayol, P. Mostafalu, M. Akbari, M. Comotto, N. Annabi, M. Ghaderi, S. Sonkusale, M. R. Dokmeci, A. Khademhosseini, Advanced Healthcare Materials 2016, 5, 175.

[244] R. C. Webb, A. P. Bonifas, A. Behnaz, Y. Zhang, K. J. Yu, H. Cheng, M. Shi, Z. Bian, Z. Liu, Y.-S. Kim, W.-H. Yeo, J. S. Park, J. Song, Y. Li, Y. Huang, A. M. Gorbach, J. A. Rogers, Nature Materials 2013, 12, 938.

[245] R. C. Webb, R. M. Pielak, P. Bastien, J. Ayers, J. Niittynen, J. Kurniawan, M. Manco, A. Lin, N. H. Cho, V. Malyrchuk, G. Balooch, J. A. Rogers, PLOS ONE 2015, 10, $\mathrm{e} 0118131$.

[246] J.-H. Park, J.-W. Lee, Y.-C. Kim, M. R. Prausnitz, International Journal of Pharmaceutics 2008, 359, 94.

[247] S. F. Nadler, K. Weingand, R. J. Kruse, Pain physician 2004, 7, 395.

[248] H. Cramer, C. Baumgarten, K.-E. Choi, R. Lauche, F. J. Saha, F. Musial, G. Dobos, European Journal of Integrative Medicine 2012, 4, e371.

[249] S. K. Barua, M. Z. A. Chowdhury, Chattagram Maa-O-Shishu Hospital Medical College Journal 2014, 13, 60.

[250] H. Lee, Medical Science Monitor 2013, 19, 661.

[251] S. Michlovitz, L. Hun, G. N. Erasala, D. A. Hengehold, K. W. Weingand, Archives of Physical Medicine and Rehabilitation 2004, 85, 1409.

[252] H. Zhai, R. Wang, X. Wang, Y. Cheng, L. Shi, J. Sun, Nano Research 2016, 9, 3924.

[253] T. Q. Trung, N.-E. Lee, Journal of Materials Chemistry C 2017, 5, 2202. 


\section{WILEY-VCH}

[254] W. Jiang, D. Niu, H. Liu, C. Wang, T. Zhao, L. Yin, Y. Shi, B. Chen, Y. Ding, B. Lu, Advanced Functional Materials 2014, 24, 7598.

[255] R. H. Baughman, C. Cui, A. A. Zakhidov, Z. Iqbal, J. N. Barisci, G. M. Spinks, G. G. Wallace, A. Mazzoldi, D. D. Rossi, A. G. Rinzler, O. Jaschinski, S. Roth, M. Kertesz, Science 1999, 284, 1340.

[256] T. Kimura, Y. Umehara, F. Kimura, Soft Matter 2012, 8, 6206.

[257] R. V. Martinez, A. C. Glavan, C. Keplinger, A. I. Oyetibo, G. M. Whitesides, Advanced Functional Materials 2014, 24, 3003.

[258] S.-E. Zhu, R. Shabani, J. Rho, Y. Kim, B. H. Hong, J.-H. Ahn, H. J. Cho, Nano Lett. 2011, 11, 977.

[259] L. Chen, M. Weng, W. Zhang, Z. Zhou, Y. Zhou, D. Xia, J. Li, Z. Huang, C. Liu, S. Fan, Nanoscale 2016, 8, 6877.

[260] W. Zhang, M. Weng, P. Zhou, L. Chen, Z. Huang, L. Zhang, C. Liu, S. Fan, Carbon 2017, 116,625 .

[261] S. Walia, R. Gupta, K. D. M. Rao, G. U. Kulkarni, ACS Applied Materials \& Interfaces 2016, 8, 23419.

[262] K. C. Heo, S. H. Yu, J. H. Kwon, J. S. Gwag, Appl. Opt., AO 2013, 52, 8460.

[263] P. Won, J. J. Park, T. Lee, I. Ha, S. Han, M. Choi, J. Lee, S. Hong, K.-J. Cho, S. H. Ko, Nano Letters 2019, 19, 6087.

[264] L. Ou, B. Song, H. Liang, J. Liu, X. Feng, B. Deng, T. Sun, L. Shao, Particle and Fibre Toxicology 2016, 13, DOI 10.1186/s12989-016-0168-y.

[265] M. Charehsaz, S. Coskun, H. E. Unalan, R. Reis, S. Helvacioglu, A. K. Giri, A. Aydin, Toxicological \& Environmental Chemistry 2017, 99, 837.

[266] S. G. Lehmann, D. Toybou, A.-E. Pradas del Real, D. Arndt, A. Tagmount, M. Viau, M. Safi, A. Pacureanu, P. Cloetens, S. Bohic, M. Salomé, H. Castillo-Michel, B. Omaña-Sanz, A. Hofmann, C. Vulpe, J.-P. Simonato, C. Celle, L. Charlet, B. Gilbert, Proceedings of the National Academy of Sciences 2019, 116, 14893.

[267] D. Mohanta, S. Patnaik, S. Sood, N. Das, Journal of Pharmaceutical Analysis 2019, DOI 10.1016/j.jpha.2019.04.003.

[268] Y. Tabei, A. Sonoda, Y. Nakajima, V. Biju, Y. Makita, Y. Yoshida, M. Horie, Metallomics 2015, 7, 816. 